21 Correspondence to: Müller-Reichert ${ }^{1}$ USA

T. Müller-Reichert

\title{
Three-dimensional structure of the kinetochore- fibers in human mitotic spindles
}

Robert Kiewisz ${ }^{1 \dagger}$, Gunar Fabig ${ }^{1}$, William Conway ${ }^{2}$, Daniel Needleman ${ }^{2,3,4,5}$, and Thomas

${ }^{1}$ Experimental Center, Faculty of Medicine Carl Gustav Carus, Technische Universität Dresden, 01307 Dresden, Germany

${ }^{2}$ Department of Physics, Harvard University, Cambridge, MA 02138, USA

${ }^{3}$ Department of Molecular and Cellular Biology, Harvard University, Cambridge, MA 02138,

${ }^{4} J o h n$ A. Paulson School of Engineering and Applied Sciences, Harvard University, Cambridge, MA 02138, USA

${ }^{5}$ Center for Computational Biology, Flatiron Institute, New York, NY 10010, USA

†Current address of R. Kiewisz: Simons Machine Learning Center, New York Structural Biology Center, New York, NY 10027, USA

E-mail: mueller-reichert@tu-dresden.de

Phone: +493514586442 


\section{Abstract}

26 During cell division, kinetochore microtubules (KMTs) provide a physical linkage between the 27 spindle poles and the chromosomes. KMTs in mammalian cells are organized into bundles, 28 so-called kinetochore-fibers (k-fibers), but the ultrastructure of these fibers is currently not well characterized. Here we show by large-scale electron tomography that each k-fiber in HeLa cells in metaphase is composed of approximately nine KMTs, only half of which reach the spindle pole. Our comprehensive reconstructions allowed us to analyze the three-dimensional (3D) morphology of k-fibers in detail, and we find that they exhibit remarkable variation. Kfibers display differences in circumference and KMT density along their length, with the polefacing side showing a splayed-out appearance. We further observed that the association of KMTs with non-KMTs predominantly occurs in the spindle pole regions. Our 3D reconstructions have implications for models of KMT behavior and k-fiber self-organization as covered in a parallel publication applying complementary live-cell imaging in combination with biophysical modeling (Conway et al., 2021). The presented data will also serve as a resource for further studies on mitosis in human cells. 


\section{Introduction}

Chromosome segregation during cell division is carried out by microtubule (MT)-based spindles (Anjur-Dietrich et al., 2021; Mclntosh et al., 2013; Oriola et al., 2018; Prosser and Pelletier, 2017). While mitotic spindles can contain thousands of MTs, only a fraction of those highly dynamic filaments is associated with the kinetochores. These MTs are called kinetochore microtubules (KMTs) and function to establish a physical connection between the chromosomes and the spindle poles (Flemming, 1879; Khodjakov et al., 1997; Maiato et al., 2004; Musacchio and Desai, 2017; Rieder, 1981; Rieder and Salmon, 1998).

Mitotic KMTs show various patterns of organization in different species. Single KMTs are connected to the kinetochores, in budding yeast (Winey et al., 1995), while multiple KMTs are connected to dispersed kinetochores in nematodes (Fabig et al., 2020; Redemann et al., 2017). Multiple KMTs are also connected to kinetochores in human cells. KMTs in human cells are organized in bundles, termed "kinetochore (k)-fibers", which are attached to a single spot on each chromosome (Begley et al., 2021; Godek et al., 2015; Inoué, 1953; Metzner, 1894; Mitchison and Kirschner, 1984; O'Toole et al., 2020; Schmidt, 1939).

The regulation of KMT dynamics in mitotic spindles has been studied in great detail in a number of different systems, including the early Caenorhabditis elegans embryo, Xenopus egg extracts and mammalian tissue culture cells (DeLuca et al., 2006; Dumont and Mitchison, 2009; Farhadifar et al., 2020; Inoué and Salmon, 1995; Kuhn and Dumont, 2019; Long et al., 2020). Our understanding of the ultrastructure of KMTs in mammalian k-fibers, however, is rather limited due to a low number of three-dimensional (3D) studies on spindle organization. Earlier studies applied several techniques. Some studies used serial thin-section transmission electron microscopy (TEM) (McDonald et al., 1992; McEwen et al., 1998, 1997) and partial 3D reconstruction by electron tomography. Other studies used serial block-face imaging by scanning electron microscopy to analyze the ultrastructure of mitotic spindles (Hoffman et al., 2020; Nixon et al., 2017, 2015). However, these prior studies did not present comprehensive $3 \mathrm{D}$ reconstructions of mammalian mitotic spindles. Nevertheless, by applying serial thinsection TEM it was reported that k-fibers in PtK 1 cells are composed of about $20 \mathrm{KMTs}$ (McDonald et al., 1992; McEwen et al., 1997). In contrast, tomographic analysis of RPE-1 cells revealed $12.6 \pm 1.7 \mathrm{KMTs}$ per k-fiber (O'Toole et al., 2020). This variation in the reported numbers of KMTs per k-fiber as well as a lack of complete 3D models of human mitotic length distribution in the context of whole mitotic spindles in human tissue culture cells. 
Two opposing models of k-fiber organization can be drawn. First, a direct connection between kinetochores and spindle poles can be considered (Fig. 1A), in which all KMTs in a given kfiber have approximately the same length and are rigidly connected (Rieder, 1981; Ris and Witt, 1981). As for the length of the KMTs, previous electron microscopic analyses of chemically fixed PtK 1 and RPE-1 cells have shown short KMTs associated with kinetochores (McDonald et al., 1992; Sikirzhytski et al., 2018). This suggested that not all KMTs in the kfiber are of the same length, implying that not all KMTs are associated with the spindle poles. Second, an indirect connection may be considered (Fig. 1B). In such a model, none of the KMT minus ends would be directly associated with the spindle poles, thus KMTs would show differences in their length and connect to the poles purely by interactions with non-KMTs in the spindle (Sikirzhytski et al., 2018, 2014). However, the kinetochore-to-spindle pole connection in human cells may be neither direct nor indirect, thus showing a semi-direct pattern of connection, in which only some of the KMTs of each k-fiber is associated with the spindle pole while others are not. Previously, we have shown such a semi-direct pattern of KMT anchoring into the spindle network for the first embryonic mitosis in the nematode Caenorhabditis elegans (Redemann et al., 2017). Some KMTs in this system are indeed directly associated with the spindle poles, while others are not.

Here we aimed to determine the number and the length of KMTs, and the positioning of their putative minus ends in human k-fibers. Focusing on metaphase, we applied serial-section electron tomography to produce large-scale reconstructions of entire mitotic spindles of HeLa cells and developed software tools for a quantitative in-depth analysis of both KMTs and nonKMTs. We found that k-fibers in HeLa cells are composed of approximately nine KMTs of variable length. These k-fibers display a previously unexpected variable morphology: they did not resemble an organized and compacted structure throughout their whole length. For better visualization of spindle and $\mathrm{k}$-fiber morphology, we introduce a new 3D visualization tool that allows the interested reader to interactively display the 3D data 


\section{K-fibers are composed of approximately nine KMTs}

102

103

104

105

106

107

108

109

110

For our large-scale analysis of mammalian k-fibers, we acquired data on metaphase spindles in HeLa cells by serial-section electron tomography (Fig. 2A-B). In three full reconstructions, we segmented the spindle poles (including the centrioles), the chromosomes and all MTs (Fig. 2C-E). These metaphase spindles were composed of approximately 6300 MTs (6278 \pm 1614 MTs, mean \pm STD; Table 1) and had an average pole-to-pole length of $9.0 \pm 1.7 \mu \mathrm{m}$ (mean \pm STD; Figure 2-figure supplement 1A-B).

We then annotated the KMTs in our reconstructions based on the association of the putative MT plus ends with kinetochores. KMTs that were arranged in parallel and made end-on contact at a single 'spot' on the chromosomes were designed as being part of the same kfiber (Figure 1-movie supplement 1-3). In our three data sets, we identified between 90 and 110 k-fibers per cell, which included on average $859 \pm 218 \mathrm{KMTs}$ (mean \pm STD; $n=3$; Fig. 2F-H; Figure 1-movie supplement 4-6) in each spindle. Thus, only $\sim 14 \%$ of all MTs in the reconstructed spindles were KMTs, which corresponds to $\sim 24 \%$ of tubulin mass (Conway et al., 2021). The identified KMTs displayed open flared ends at the kinetochore (Fig. 2-figure supplement 2), consistent with previous observations of the morphology of KMT plus ends (Mclntosh et al., 2013; O'Toole et al., 2003). We took advantage of these extracted k-fibers to further analyze the distance between the sister kinetochores in each data set by measuring the median position of the KMT plus ends (Figure 2-figure supplement 1C-D). The average distance between sister kinetochores was $1.1 \pm 0.2 \mu \mathrm{m}$.

Next, we extracted individual k-fibers from our full 3D reconstructions to visualize their overall morphology (Fig. 3A and Figure 3-movie supplements 1-6). The individual k-fibers showed remarkable differences in their overall shapes. Some k-fibers were rather straight, while others were very curved. In addition, $\mathrm{k}$-fibers showed variability in their compactness as defined by the density of KMTs in a given k-fiber). At the site of KMT attachment to the chromosomes, kfibers showed a compacted appearance, while k-fibers were considerably splayed out at the site facing the spindle poles. Moreover, some pole proximal KMT ends extend beyond the position of the spindle poles.

We next investigated the number of KMTs per kinetochore (Fig. 3B and Figure 3-figure supplement $1 \mathrm{~A}$ ) and found that the k-fibers were composed of $8.5 \pm 2.2 \mathrm{KMTs}$ (mean \pm STD, $\mathrm{n}=293$ ). Previously published data indicated that the number of KMTs is correlated with kinetochore size (McEwen et al., 1998) so that larger kinetochores are associated with more 
KMTs. Therefore, we estimated the size of the kinetochores in our three reconstructed spindles by determining the cross-sectional area of the k-fibers close to the kinetochores. The average kinetochore area was $0.07 \pm 0.05 \mu \mathrm{m}^{2}$ (mean \pm STD; Figure 3-figure supplement 1C-D; while the Poisson correlation coefficient between the number of KMTs and kinetochore area was 1.96 with $p=1.91 \times 10^{-8}$ ). This is in line with an observation on the kinetochore area in HeLa cells as analyzed by light microscopy (Cherry et al., 1989). Moreover, we also observed a packing density of KMTs at the kinetochore of $0.07 \pm 0.05 \mathrm{KMTs} / \mu^{2}$ (mean \pm STD, $n=293$; Figure 3-figure supplement 1E; Table 3) with average center-to-center distance between KMTs of $64 \pm 19 \mathrm{~nm}$ (mean \pm STD, $\mathrm{n}=293$; Figure 3-figure supplement 1F; Table 3). Considering a MT diameter of $25 \mathrm{~nm}$ in our data sets, this corresponds to an average $40 \mathrm{~nm}$ wall-to-wall spacing between the KMTs at the kinetochore. With this observation we concluded that KMTs tend to be highly compacted at the kinetochore and that kinetochore size does not influence KMT packaging density or the distance between KMTs. We also investigated a possible influence of the inter-kinetochore distance on the number of MTs attached to the kinetochores. We plotted both the number of attached KMTs and the difference in the number of KMTs attached to sister kinetochores against the inter-kinetochore distance. We did not observe a correlation between these parameters (Fig 3C-D; Pearson's correlation coefficients were 0.04 and 0.29 ) and concluded that the inter-kinetochore distance does not play a significant role in determining the number of KMTs in metaphase.

We next sought to characterize the kinetochore-to-spindle pole connection in more detail. Therefore, we developed software to analyze the structure of KMTs in individual k-fibers (Kiewisz and Müller-Reichert, 2021). We investigated the distribution of KMT lengths and the positioning of KMT minus ends along the spindle axis (Fig. 4A). We were specifically interested in the percentage of KMT minus ends that were directly associated with the spindle poles. Similar to a previously published analysis in C. elegans (Redemann et al., 2017), we defined a cutoff distance for an MT-centrosome interaction. We used a cutoff distance of 1.53 $\mu \mathrm{m}$ which was twice the half-width of the peak of non-KMT ends near the pole (Fig. 4B, grey area). Thus, we considered KMT minus ends within $1.53 \mu \mathrm{m}$ from the center of the mother centriole to be directly attached to the pole and KMT minus ends positioned further than the $1.53 \mu \mathrm{m}$ cutoff to be indirectly attached to the pole.

We then continued by measuring the length of all KMTs in our reconstructions (Fig. 4C; Figure 4-figure supplement 1A) and observed a broad distribution of KMT lengths with an average value of $3.7 \pm 1.8 \mu \mathrm{m}$ (mean \pm STD; $n=2489$ ). This analysis suggested the existence of relatively short KMTs in $\mathrm{k}$-fibers not associated with the spindle poles. Indeed, about $21 \pm 4 \%$ of the KMTs had lengths less than $2 \mu \mathrm{m}$. Our analysis also revealed relatively long KMTs (about $35 \pm 10 \%$ ) that were longer than the half spindle length. Some of these long KMTs 
showed a pronounced curvature at their pole-facing side, thus connecting to the 'back side' of the spindle poles (see also Fig. 3A, spindle \#II). We then analyzed the distances of the KMT minus ends to the spindle poles (Fig. 4D; Figure 4-figure supplement 1B). Taking our determined cutoff value into account, we found that only about $50 \%(54.4 \pm 9.3 \%$; mean \pm STD, $n=3$ ) of the KMTs were associated with the spindle poles, while the other half of the KMTs were not (Figure 3-figure supplement 1B). This suggests that half of the KMTs are indirectly connected to the spindle poles.

We also observed that the number of KMT minus ends associated with the spindle pole was significantly greater in fibers positioned in the center compared to those at the periphery of the mitotic spindle (Figure 4-figure supplement 2). We next investigated the relative position of the KMT minus ends on the pole-to-kinetochore axis (Fig. 4E; Figure 4-figure supplement 1C). We found that KMT minus ends that were positioned within the centrosomal association zone showed a peak position close to the spindle pole center. In contrast, KMT minus ends not associated with a spindle pole did not show a preferred position but rather displayed a flat relative distribution on the pole-to-kinetochore axis. This analysis confirmed our initial visual 3D inspection of the KMTs, revealing that the k-fibers in HeLa cells are not composed of compact bundles of KMTs of the same length but rather of KMTs of different lengths. Importantly, our data analysis also showed that only half of the KMTs in k-fibers are directly associated with the spindle poles.

For comparison, we also analyzed the length distribution of non-KMTs in the spindles. NonKMTs had an average length of $2.0 \pm 1.6 \mu \mathrm{m}$ (mean \pm STD; $n=9957$; Fig. 4F; Figure 4-figure supplement 1D), with many very short MTs and fewer long MTs. In addition, $39 \pm 9 \%$ of the non-KMT minus ends were localized in the MT-centrosome association zone (Fig. 4G; Figure 4-figure supplement 1E) with the remaining $\sim 60 \%$ in the bulk of the spindle (Fig. $4 \mathrm{H}$; Figure 4-figure supplement 1F).

\section{K-fibers show a splayed-out appearance at spindle poles}

The variable morphology of k-fibers within spindles motivated us to further analyze the 3D structure of individual KMTs in the k-fibers. For this, we measured the tortuosity of individual KMTs. Tortuosity is the ratio of the total length of a curve (the spline length of a given KMT) to the distance between its ends (given here by the 3D Euclidean distance between the KMT ends). Straight KMTs will have a tortuosity of 1 while a quarter circle has tortuosity of around $\sim 1.1$ and a half-circle around $\sim 1.6$ (Fig. 5A). We aimed to measure both the global and the local tortuosity of the KMTs in our reconstructions, i.e., the tortuosity of the KMTs along their entire length and also in defined sections of $500 \mathrm{~nm}$ length along the k-fibers, respectively 
(Fig. 5B-C). Firstly, we visualized the global tortuosity of individual KMTs by applying a color code. We detected differences in the curvature of individual KMTs (Fig. 5D, and Figure 5movie supplement 1-3). For all data sets, we observed an average value of KMT tortuosity of $1.09 \pm 0.09$ (mean \pm STD, $n=2489$; Fig. $\mathbf{5 F}$ ) with $32 \pm 10 \%$ of KMTs showing a tortuosity higher than 1.1. We could also observe that straight KMTs were predominantly located in the center of the spindle with an average tortuosity of 1.07, while more curved KMTs were located more at peripheral spindle positions with an average tortuosity of 1.09 (Fig. 5E; Figure 5figure supplement 1 ). This indicates that the majority of KMTs in the spindle are rather straight and do not show a curvature that is close to a quarter of a circle. Furthermore, the tortuosity of KMTs was correlated with their length $(R=0.65 ; p=2.2 e-16$; Fig. $5 \mathbf{G})$ with short MTs being more straight and longer MTs more curved. In addition, $68 \pm 13 \%$ of the KMTs with a tortuosity higher than 1.1 were longer than the half-spindle length. Secondly, we also investigated the local tortuosity of the KMTs. (Fig. 5H). Our analysis revealed that the local tortuosity of the KMTs was correlated with the relative position of the KMT segments on the pole-to-kinetochore axis. In general, the tortuosity of KMTs was not uniform along their entire length but increased at positions closer to the spindle poles. $(R=-0.30 ; p=2.2 e-16$; Fig. 5 I $)$.

Next, we asked whether an increase in k-fiber tortuosity at the pole-facing end is reflected in the overall KMT organization, i.e., in a change in the compactness of the k-fibers along their entire length. For this, we analyzed the cross-section area of the k-fibers by determining their polygon areas (Fig. 6A, and Figure 6-figure supplement 1) and plotting these areas against the relative position on the pole-to-kinetochore axis (Fig. 6B). K-fibers had an average crosssection area of $0.07 \pm 0.11 \mu \mathrm{m}^{2}( \pm$ STD, $n=293)$. Compared to the kinetochore-facing side of the k-fibers, the polygon area was 10-fold higher at the mid-position of the k-fiber and 5-fold lower at the pole-facing end of the fibers. Moreover, at the pole-facing side, the k-fiber area showed a higher spread of the values, reflecting the splayed-out appearance of the k-fiber at the spindle poles. To further characterize the arrangement of the KMTs in the k-fibers, we also set out to measure the density of the KMTs along the length of the k-fibers (Fig. 6C). For each k-fiber, we defined a minimum circle enclosing all KMTs at the kinetochore side. We then measured the number of KMTs that were enclosed in the selected radius at positions along the k-fibers and plotted the percentage of the enclosed KMTs against the relative position along the pole-to-kinetochore axis. We observed a variation in the percentage of enclosed KMTs along the k-fiber length with the highest percentage at the kinetochore and a lower percentage in the pole-facing side of the reconstructed k-fibers (Fig. 6D). From all these analyses we concluded that k-fibers display a high tortuosity and a low density of KMTs close to the spindle poles, thus leading to an overall splayed-out appearance of the fibers at their pole-facing end. 


\section{KMTs primarily associate with non-KMTs at spindle poles}

240

241

242

243

244

245

246

247

248

249

250

251

252

253

254

255

256

257

258

259

260

261

262

263

264

265

266

267

268

269

270

271

272

273

We next investigated the pattern of association of KMTs with the neighboring non-KMTs. We were particularly interested in mapping KMT/non-KMT associations along the spindle axis. To describe this association, we considered two types of interactions between MTs. Firstly, we analyzed potential interactions between MT ends with neighboring MT lattices, which could be mediated by MT minus-end associated molecular motors, such as dynein (Tan et al., 2018) or kinesin-14 (Molodtsov et al., 2016), or by Y-tubulin (Rosselló et al., 2018). Secondly, we considered MT-MT lattice interactions, which might be established by molecular motors such as kinesin-5 (Falnikar et al., 2011). We started our analysis by investigating possible KMT minus-end associations with either non-KMT or KMT lattices. For this, we annotated all KMT minus ends in our 3D reconstructions and measured the distance of each minus end to a neighboring MT lattice (Fig. 7A). We then determined what percentage of all KMT minus ends were associated with non-KMT lattices according to selected association distances. We considered different possible association distances between KMT minus ends and the neighboring MT lattices (i.e., 25, 30, 35, 45, 50, 75 and 100 nm; Fig. 7B; Table 4 and Table 5). As expected, we observed that the number of KMT minus ends associated with adjacent MT lattices increased with an increase in the distance of association. Considering $35 \mathrm{~nm}$ as an example for a possible interaction distance between two MTs connected by a single dynein motor (Amos, 1989), we observed that only $41.4 \pm 6.3 \%$ of the KMT minus ends were associated with other MTs (for a visualization of the pattern of association see Fig. 7C; Figure 7-movie supplement 1). Moreover, of the KMT minus ends that were not associated with the spindle poles (i.e., the ones positioned farther than $1.53 \mu \mathrm{m}$ away from the centrioles) only $40.9 \pm 5.4 \%$ showed an association with other MT lattices at a given distance of $35 \mathrm{~nm}$ (Fig. 7D-E). This suggested that for this given distance of association roughly only $40 \%$ of the KMT minus ends in k-fibers were associated with the MT network. Further considering larger distances of association between KMT minus end and neighboring MTs (Fig. 7B and Fig. 7D), we also observed that not all KMT minus ends were associated with neighboring MTs even at a value of $100 \mathrm{~nm}$ (Table 4 and Table 5).

Next, we sought to map the detected associations of KMT minus ends with either KMT or nonKMT lattices within the reconstructed spindles. For this, we determined the position of such associations in half spindles and plotted the data against the relative spindle position (i.e., against the normalized spindle pole-to-kinetochore distance). We first plotted the number of KMT minus end associations with KMT lattices against the relative position on the spindle axis (Fig. 7F). As a result, associations of KMT minus ends with KMT lattices were distributed along the whole half spindle length with a preference for positions at the spindle poles. As an 
example, for a given association distance of $35 \mathrm{~nm}, 47.5 \pm 13.8 \%$ of the total number of associations were observed at the spindle poles. We then also determined the relative position of the KMT minus end associations with non-KMT lattices (Fig. 7G). Similarly, the majority of the associations of KMT minus ends with non-KMT lattices were observed at the spindle pole. Similarly, at a chosen distance of $35 \mathrm{~nm}, 51.1 \pm 14.7 \%$ of these associations were observed at the spindle poles. Thus, the spindle pole appeared as a 'hotspot' for the interaction of KMT minus ends with neighboring MT lattices.

Vice versa, we also determined the occurrence of either KMT minus or non-KMT pole-proximal ends in the vicinity of KMT lattices (Figure 7-figure supplement 1; Table 6 and Table 7). For this analysis, we assumed that the end of each non-KMT that is closer to the centrioles represents the minus end. At a distance of $35 \mathrm{~nm}$ or closer to the KMT lattice, we observed that on average $43 \pm 8 \%$ of KMTs associated with either KMT or non-KMT minus ends, with the majority of associations with non-KMT minus ends (73 $\pm 4 \%$ ). Moreover, we also determined the relative position of these associations on the spindle axis. Again, the majority of KMT lattices (59.8 $\pm 6.7 \%$ ) associated with other MT minus ends were preferentially found at spindle poles. In contrast, only $39.1 \pm 4.6 \%$ of non-KMTs associated with other MT minus ends were found at the poles. Again, this analysis indicated that the interaction of KMTs with other MTs preferentially takes place at the spindle poles regardless of the association distance (Figure 7-figure supplement 1 ).

Next, we also considered the length of these MT-MT associations in the spindles. For such a length analysis, we identified individual associations of a minimal length of $20 \mathrm{~nm}$ and determined their position and number along the spindle axis (Fig. 8A). The minimal length of $20 \mathrm{~nm}$ was selected as a fixed distance step size to minimize computational efforts and unify all analyses. In addition, we also varied the distance between associated MTs by choosing values of $25,30,35,45$ and $50 \mathrm{~nm}$. Firstly, we analyzed the association of KMTs with other MTs in the spindle by plotting the number of associations (i.e., the number of the $20 \mathrm{~nm}$ intervals) against the relative position on the normalized spindle axis. For all considered distances between MTs, we observed peaks of associations at positions close to the spindle poles and a drastic decline in the number of associations at positions in the middle of the spindle (Fig. 8B-C; Figure 8-movie supplement 1). Secondly, we also analyzed the association of non-KMTs with other MTs. In contrast to the previous analysis, we found that these interactions were spread over the entire length of the spindle without any 'hotspots' of associations for small distances between 25 and 35 nm between MTs (Fig. 8D-E; Figure 8movie supplement 2). In fact, similar distribution patterns could be observed for all chosen association distances. This indicated to us that the observed MT-MT association patterns for 
309 both KMTs and non-KMTs were not dependent on the chosen association distances. We also 310 concluded from these findings that KMTs and non-KMTs differ in their spatial pattern of MT311 MT association, with KMTs strongly coupling with neighboring MT at the spindle poles, and 312 non-KMTs showing a more even distribution of MT-MT associations along the spindle axis.

313 We were also interested in how the distribution pattern of MT-MT interaction changes in 314 relation to the position in the spindle. With our high-resolution 3D datasets covering all MTs in 315 the spindle, we decided to investigate the number and the length of associations for both KMTs 316 and non-KMTs as a function of the distance between MTs. Firstly, we plotted the number of 317 KMTs against the number of associations and also against the length of associations (Figure 318 8-supplement 1). In general, with an increase in the distance between MTs, KMTs showed 319 an increase in the number and also in the average length of interactions (Table 8 and Table 320 9). For a given MT-MT distance of $35 \mathrm{~nm}$, the number of associations was $14 \pm 6( \pm S T D, n=$ $3212949)$ with an average MT length of association of $163 \pm 70 \mathrm{~nm}( \pm S T D, n=2949)$. Secondly, we also analyzed the association of non-KMTs with spindle MTs. Non-KMTs showed a similar pattern of increase in the number and length of associations with increasing distances between individual MTs. For a distance of $35 \mathrm{~nm}$, the number of associations was $10 \pm 6$ $( \pm S T D, n=9957)$ with an average length of association of $128 \pm 75 \mathrm{~nm}( \pm S T D, n=9957)$. With an increase in the distance between MTs, we observed that KMTs tend to show a higher number of associations and a higher average length of associations compared to non-KMTs. Importantly, these results were consistent for all selected association distances, thus confirming that both KMTs and non-KMTs show distinct patterns of MT-MT associations within the metaphase spindle. 
332 The ultrastructure of human k-fibers was insufficiently described prior to this study on the 3D

333 organization of metaphase spindles. Large-scale reconstruction by serial-electron tomography

334 allowed quantitative analyses of KMT organization in individual k-fibers in the context of whole 335 spindles.

\section{Type of kinetochore-to-spindle pole connection}

337 Electron tomography revealed that on average nine KMTs are attached to each kinetochore 338 in HeLa cells in metaphase. This result differs from previous observations in PtK ${ }_{1}$ cells 339 (McEwen et al., 1997; O'Toole et al., 2020). In this marsupial cell line, about 20 KMTs were 340 reported to connect to the kinetochores. This difference in the number of attached KMTs could 341 be related to kinetochore size. As previously observed by light microscopy, kinetochores in 342 HeLa cells have about half of the size of kinetochores in PtK 1 cells (Cherry et al., 1989). 343 Similarly, kinetochore size in PtK $_{1}$ cells was $0.157 \pm 0.045 \mu \mathrm{m}^{2}$ (mean \pm STD; (McEwen et al., 344 1997)) as observed by electron tomography, whereas kinetochores in HeLa cells, as 345 determined indirectly by electron tomography in this study, have an estimated size of about $3460.07 \pm 0.05 \mu \mathrm{m}^{2}$ (mean \pm STD). Possibly, the kinetochore area might indirectly define the size and/or the number of the available free binding sites for MTs (Drpic et al., 2018; Monda and

348 Cheeseman, 2018). The size of the kinetochore appears to be different in different species 349 (Campbell et al., 2019).

We also observed that the connection between kinetochores and the spindle poles is not direct. Textbook models of k-fibers often show KMTs of equal length that are organized in parallel, giving the impression of 'compact rods' connecting kinetochores and spindle poles. This view of the mammalian k-fiber is not consistent with our tomographic data, as k-fibers in our reconstructions show remarkable morphological variability in their circumference along their length (Fig. 9A). KMTs in our reconstructed k-fibers are also of different lengths, confirming previous observations (McDonald et al., 1992; O'Toole et al., 2020). In fact, the kfibers have many short KMTs (less than $1.53 \mu \mathrm{m}$ in length: see Fig. 4C) with their minus ends not positioned near the spindle pole. On the other hand, the pole-to-kinetochore linkage in HeLa cells is also not entirely indirect, as we could only find a small percentage of k-fibers in our reconstructions (about 5\%; Figure 3-figure supplement 1B) that did not show any KMT associated with the corresponding spindle pole. Thus, our tomographic analysis suggests a semi-direct connection, in which at least one KMT of the k-fibers is directly connected to the poles, while the other KMTs of the fiber are indirectly linked to non-KMTs (Fig. 9B). 


\section{KMT organization and interaction with non-KMTs}

365 The length distribution of KMTs in HeLa cells shows striking similarities to the distribution observed in the early C. elegans embryo (Redemann et al., 2017). Both human KMTs attached to monocentric kinetochores and also nematode KMTs associated with dispersed holocentric kinetochores show a rather flat length distribution with a smooth cut-off and a rather low number of both very short and very long KMTs. In contrast, non-KMTs in both systems show an exponential length distribution with a very high occurrence of very short microtubules (around $64 \%$ of the MTs in HeLa cells were less than $2 \mu \mathrm{m}$ ). This argues that KMTs in both spindles have distinct properties different from non-KMTs.

We observed different association patterns for KMTs versus non-KMTs. While both KMTs and non-KMTs showed a clear correlation in the number and the average length of associations (Figure 8-figure supplement 1), both MT populations showed differences in the position of these associations. KMTs showed a high tendency to associate with non-KMTs at the spindle poles, and this was independent of the chosen association distance. Our results strongly suggest that KMTs preferably associate with other MTs at the spindle poles, thus achieving an anchoring into the spindle network as previously observed in the early $C$. elegans embryo (Redemann et al., 2017). This anchoring might be promoted by the observed splaying of the k-fibers.

In contrast, non-KMTs showed a flat pattern of interaction with other MTs at association distances of 25 and $35 \mathrm{~nm}$. Moreover, an increase in the association distance from $35 \mathrm{~nm}$ to $50 \mathrm{~nm}$, showed a higher tendency of non-KMTs to associate with MTs in the center of the spindle. At the same time, we observed a decrease in the number of associations of KMTs with the MT network at the same position. This is very likely related to the organization of interpolar MTs in the center of the spindle (Figure 7-figure supplement 1F) (Kajtez et al., 2016; Mastronarde et al., 1993; Vukušić et al., 2017).

By visual inspection of our reconstructed metaphase spindles, we could indeed observe an apparent association of KMTs with non-KMTs. Our data suggest a stochastic distribution of an association of k-fibers with non-KMTs. However, the 3D organization of interdigitating MTs in the mitotic spindle will be subject to a separate study.

In a parallel study, we have combined our 3D reconstructions with additional live-cell imaging and stochastic simulation (Conway et al., 2021). Therein, we provide evidence for a guided outgrowth of KMTs emanating from kinetochores and present a biophysical model in which the spindle apparatus is considered as a liquid crystal constraining the path of KMT outgrowth towards the spindle poles by neighboring non-KMTs. 


\section{Experimental procedures}

399

400

401

402

403

404

405

406

407

408

409

410

411

412

413

414

415

416

417

418

419

420

421

422

423

424

425

426

427

428

429

\section{Cultivation of cells}

HeLa (Kyoto) cells were grown in Dulbecco's Modified Eagle's Medium (DMEM) supplemented with $10 \%$ fetal bovine serum (FBS) and 100 units $/ \mathrm{ml}$ of penicillin/streptomycin (Pen/Strep). Flasks were placed in a humidified incubator at $37^{\circ} \mathrm{C}$ with a supply of $5 \% \mathrm{CO}_{2}$. For electron microscopy, cells in mitosis were enriched by applying the shake-off technique (Kiewisz et al., 2021). Flasks with cell confluency of $60-80 \%$ were shaken against the laboratory bench. The medium with detached cells was then collected, centrifuged at $1200 \mathrm{rpm}$ for 3 min at room temperature, and resuspended in $1 \mathrm{ml}$ of pre-warmed DMEM medium.

\section{Electron tomography}

\section{Specimen preparation for electron microscopy}

Cultures enriched in mitotic HeLa cells were further processed for electron microscopy essentially as described (Guizetti et al., 2011; Kiewisz et al., 2021). Briefly, sapphire discs with a diameter of $6 \mathrm{~mm}$ were cleaned in Piranha solution $\left(1: 1 \mathrm{H}_{2} \mathrm{SO}_{4}\right.$ and $\left.\mathrm{H}_{2} \mathrm{O}_{2}, \mathrm{v} / \mathrm{v}\right)$, coated with poly-L-lysine $\left(0.1 \%\right.$ in $\left.\mathrm{dd}_{2} \mathrm{O}, \mathrm{w} / \mathrm{v}\right)$ and dried for $2 \mathrm{hrs}$ at $60^{\circ} \mathrm{C}$. Furthermore, the discs were coated with fibronectin (1:10 dilution in $1 \times$ PBS, v/v) for 2 hrs and were stored in a humidified incubator until further used. The sapphire discs were then placed into custom-designed 3Dprinted incubation chambers (Kiewisz et al., 2021). Subsequently, cells were seeded on the coated sapphire discs and incubated for $10 \mathrm{~min}$ in a humidified incubator at $37^{\circ} \mathrm{C}$ supplied with $5 \% \mathrm{CO}_{2}$. This allowed the mitotic cells to re-attach to the surface of the coated sapphire discs and continue to divide.

High-pressure freezing and freeze substitution

Cells were cryo-immobilized using an EM ICE high-pressure freezer (Leica Microsystems, Austria). For each run of freezing, a type-A aluminum carrier (Wohlwend, Switzerland) with the $100 \mu \mathrm{m}$-cavity facing up was placed in the specimen loading device of the EM ICE system. The cavity of the type A carrier was then filled with $5 \mu$ l of DMEM containing an additional $10 \%$ BSA, and the 'sandwich' was immediately closed by placing a $6 \mathrm{~mm}$-sapphire disc with attached cells facing down. Finally, a spacer ring was mounted on top of the assembled sample 'sandwich' and the freezing was started. Samples were frozen under high pressure ( $\sim 2000$ bar) with a cooling rate of $\sim 20,000{ }^{\circ} \mathrm{C} / \mathrm{s}$ (Reipert et al., 2004). Frozen samples were then opened under liquid nitrogen and transferred to cryo-vials filled with anhydrous acetone containing $1 \%(\mathrm{w} / \mathrm{v})$ osmium tetroxide (EMS, USA) and $0.1 \%(\mathrm{w} / \mathrm{v})$ uranyl acetate 
430 (Polysciences, USA). Freeze substitution was performed in either a Leica AFS or a Lecia AFS

431 II (Leica Microsystems, Austria). Samples were kept at $-90^{\circ} \mathrm{C}$ for $1 \mathrm{~h}$, warmed up to $-30^{\circ} \mathrm{C}$ with

432 increments of $5{ }^{\circ} \mathrm{C} / \mathrm{h}$, kept for $5 \mathrm{hrs}$ at $-30^{\circ} \mathrm{C}$, and then warmed up to $0^{\circ} \mathrm{C}$ (increments of 5

$433{ }^{\circ} \mathrm{C} / \mathrm{h}$ ). Finally, samples were allowed to warm up to room temperature. After freeze

434 substitution, samples were washed three times with pure anhydrous acetone and infiltrated

435 with Epon/Araldite (EMS, USA) using increasing concentrations of resin (resin:acetone: 1:3,

$4361: 1,3: 1$, then pure resin) for $1 \mathrm{hrs}$ each step at room temperature (Muller-Reichert et al., 2003).

437 Samples were infiltrated with pure resin overnight and then embedded by using commercial 438 flow-through chambers (Leica, Vienna, Austria) designed for sapphire discs of a diameter of $4396 \mathrm{~mm}$. Samples were polymerized at $60^{\circ} \mathrm{C}$ for $36 \mathrm{hrs}$.

\section{Pre-selection of staged cells}

441 To select cells in metaphase, resin-embedded samples were pre-inspected using an Axiolab 442 RE upright brightfield microscope (Zeiss, Oberkochen, Germany) with a 5x and a 40x objective 443 lens (Zeiss, Oberkochen, Germany). Selected cells in metaphase were sectioned using an 444 EM UC6 ultramicrotome (Leica Microsystems, Austria). Ribbons of semi-thick ( $300 \mathrm{~nm})$ serial 445 sections were collected on Formvar-coated copper slot grids, post-stained with $2 \%(w / v)$ 446 uranyl acetate in $70 \%(\mathrm{v} / \mathrm{v})$ methanol, followed by $0.4 \%(\mathrm{w} / \mathrm{v})$ lead citrate (Science Services, 447 USA) in double-distilled water. In addition, $20 \mathrm{~nm}$ colloidal gold (British Biocell International, 448 UK) was attached to the serial sections, serving as fiducial markers for subsequent electron 449 tomography. The selected cells were then pre-inspected at low magnification ( 2900x) using 450 either an EM906 (Zeiss, Oberkochen, Germany) or a TECNAI T12 Biotwin (ThermoFisher 451 Scientific, Eindhoven, The Netherlands) transmission electron microscope operated at $80 \mathrm{kV}$ 452 or $120 \mathrm{kV}$, respectively.

\section{Acquisition and calculation of tomograms}

454 Serial sections of the selected cells were then transferred to a TECNAI F30 transmission 455 electron microscope (ThermoFisher Scientific, Eindhoven, The Netherlands) operated at 300 $456 \mathrm{kV}$ and equipped with a US1000 CCD camera (Gatan, USA). Using a dual-axis specimen 457 holder (Type 2040, Fishione, Export, PA, USA), tilt series were acquired from $-65^{\circ}$ to $+65^{\circ}$ 458 with $1^{\circ}$ increments at a magnification of $4700 \mathrm{x}$ and a final pixel size of $2.32 \mathrm{~nm}$ applying the 459 SerialEM software package (Mastronarde, 2005, 2003). For double-tilt electron tomography, 460 the grids were rotated for 90 degrees and the second tilt series were acquired using identical 461 microscope settings (Mastronarde, 1997). The tomographic A- and B-stacks were combined 462 using IMOD (Kremer et al., 1996; Mastronarde and Held, 2017). For each spindle 463 reconstruction, montages of $2 \times 3$ frames were collected. Depending on the orientation of the 
spindles during the sectioning process, between 22 to 35 serial sections were used to fully reconstruct the volumes of the three selected spindles (Table 8).

As previously published (Redemann et al., 2014; Weber et al., 2012) MTs were automatically segmented using the ZIBAmira (Zuse Institute Berlin, Germany) software package (Stalling et al., 2005). After manual correction of MT segmentation, the serial tomograms of each recorded cell were stitched using the segmented MTs as alignment markers (Lindow et al., 2021).

471 Following this pipeline of data acquisition and 3D reconstruction, three complete models of

472 HeLa cells in metaphase were obtained.

\section{Quantification of tomographic data}

474 For the preparation of the reconstructed mitotic spindles for further quantitative analysis, the 475 ZIBAmira (Zuse Institute Berlin, Germany) software package was used (Stalling et al., 2005). 476 In addition, an automatic spatial graph analysis (ASGA) software tool was created for the 477 quantification of the tomographic data (Kiewisz and Müller-Reichert, 2021). In detail, we 478 analyzed the length and minus end distribution of KMTs. Furthermore, we quantified the 479 position of each k-fiber in the mitotic spindles followed by an analysis of the tortuosity, the 480 cross-section area, the shape and the density of k-fibers.

\section{$481 \quad$ Staging of spindles}

482 For a precise staging of the three reconstructed metaphase spindles, we determined the inter483 kinetochore distance. For this, the closest neighboring sister kinetochores were determined. 484 The center of each kinetochore was then defined as a median position of all KMT plus ends 485 associated with each selected kinetochore, and the inter-kinetochore distance was then 486 calculated as the 3D Euclidean distance between the defined median centers of each 487 kinetochore pair. For each mitotic spindle, the inter-kinetochore distance is given as the mean 488 value (mean \pm standard deviation, STD). As an additional criterion for mitotic staging, the pole489 to-pole distances were measured. For this, we measured the 3D Euclidean distance between 490 the centers of the manually segmented mother centrioles in each data set. This read-out was 491 used to determine the spindle size at metaphase.

493 MTs with their putative plus end associated with the chromosomes were defined as KMTs. 494 Characteristically, these KMTs showed a parallel arrangement at the site of attachment to the 495 chromosomes. Unfortunately, identification of individual kinetochores in our electron 
tomograms was hindered by the fact that prominent electron-dense monopolar KMT attachment sites, as described previously for conventionally fixed cells (McEwen et al., 1998), were not always clearly visible after cryo-fixation by high-pressure freezing. All other MTs in the 3D reconstructions were classified as non-KMTs.

MT - centrosome interaction area estimation

501 The KMT minus end distance to the closest spindle pole was calculated as a 3D Euclidean

502 distance. The border of the spindle pole (the MT - centrosome interaction area) was estimated 503 from all data sets by defining a distribution peak of non-KMT minus end distance to a spindle 504 pole. The centrosome interaction area was then defined as a double of half-width from the 505 distribution peak.

507 The full length of each reconstructed KMT and non-KMTs was measured, and the average is 508 given for each data set (mean \pm STD). We also analyzed the percentage of short versus long 509 KMTs. For each data set, short KMTs were defined as those shorter than $1.53 \mu \mathrm{m}$ in length. 510 This threshold was chosen based on the MT - centrosome interaction area. The long KMTs 511 were identified as KMTs longer than a half-spindle length for each given data set.

\section{Position of MT minus ends}

513 To analyze the position of the KMT minus ends in the metaphase spindles, the 3D Euclidean 514 distance of each KMT minus end to the closest spindle pole (i.e., to the center of the mother 515 centriole) was determined. In addition, the relative position of KMT minus ends along the pole516 to-kinetochore axis was calculated. The relative position of each minus end is given as the 517 normalized position between the kinetochore (position =1) and mother centriole (position = 0) 518 along the spindle axis. The distribution of relative positions of KMT minus ends (mean, \pm STD) 519 is given for each data set. The number of KMT minus ends not associated with the spindle 520 pole were defined as minus ends detected further than the calculated MT - centrosome interaction area.

523 KMT minus ends association with other MT lattices was calculated as a 3D Euclidean 524 distance. For that firstly, KMT minus ends were identified as KMT ends that were closer to one 525 of the spindle poles. Finally, a possible association between KMT ends and other MT lattices was measured by calculating a 3D Euclidean distance between KMT ends and every MT lattice in the reconstructed spindle. The interaction between KMT minus ends and MT lattice was identified if KMT minus ends were found in interaction distance to any MT lattice. The 
529 interaction distance was defined as 25, 30, 35, 45, 50, 75 and $100 \mathrm{~nm}$. For visualization, each

$530 \mathrm{KMT}$ was labeled based on the type of detected interaction (no interaction, interaction with

$531 \mathrm{KMT}$, interaction with non-KMT). The percentage number of KMT with any interaction was

532 measured and the average value for all data sets is given (mean \pm STD).

533 Additionally, to identify possible MT minus ends association with KMT lattices, the 3D 534 Euclidean distances of the MT minus ends to KMT lattices were calculated. The association 535 between KMT lattices and MT minus ends was identified if MT minus ends were found in 536 interaction distance to KMT lattices. The interaction distance was defined as $25,30,35,45$, 53750,75 and $100 \mathrm{~nm}$. The percentage number of KMT with any interaction was measured and 538 the average from all datasets is given (mean \pm STD). Moreover, to calculate the position on 539 the KMT where the interaction occurred, a relative position of MT minus ends on the pole-to540 kinetochore axis was calculated. The relative position of each minus end is given as the 541 normalized position between the kinetochore (position =1) and mother centriole (position = 0 ) 542 along the spindle axis.

\section{Defining kinetochore position}

544 In order to determine the position of the k-fiber in the mitotic spindle, a position model was created based on the kinetochore localization on the metaphase plate. First, kinetochores were assigned to the outer, middle, or inner regions of the metaphase plate. For that, all kinetochores were projected in a 2D space using an X/Z spindle axis, and an ellipse was fitted into all projected kinetochores in a way that a semi-major axis was defined as the length of the furthest kinetochore on the X-axis, and a semi-minor axis defined as the length of the furthest kinetochore on the Z-axis. The fitted ellipse was divided into three regions ranging from $100-75 \%$ (outer region), from 75 - $50 \%$ (middle region) and from 50 - 0\% (inner region). Kinetochores with associated k-fibers were then assigned to these regions.

554 For the analysis of KMT tortuosity, the ratio of the KMT spline length and the 3D Euclidean distance between the plus and the minus end for each KMT was measured. The distribution of KMTs tortuosity (mean, \pm STD) is given. In addition, the correlation of the KMT tortuosity with its length is given as a fitted polynomial line calculated as local polynomial regression by the locally estimated scatterplot smoothing "loess" method. A confidence interval for the created polynomial line was calculated with the t-based approximation which is defined as the overall uncertainty of how the fitted polynomial line fits the population of all data points. Local polynomial regressions and confidence intervals for all data sets were calculated using the stat 4.0.3 R library (R Core Team, 2021). 
564 For the calculation of the local tortuosity, each KMT was subsampled with segments of a 565 length of $500 \mathrm{~nm}$. Both the tortuosity and the relative position along the pole-to-kinetochore axis were measured for each segment. In addition, the correlation of local KMT tortuosity against the relative position is given. Local polynomial regressions and confidence intervals for all data sets were calculated using the stat 4.0.3 R library (R Core Team, 2021).

569

570

571

572

573

574

575

576

577

578

579

580

581

582

583

584

585

586

587

588

589

590

591

592

593

594

\section{Polygon cross-section area of $k$-fibers}

The cross-section area was calculated every $500 \mathrm{~nm}$ along each k-fiber. For each defined cross-section, KMTs position was mapped on a $2 \mathrm{D}$ plane, and a polygon shape of a k-fiber cross-section was calculated based on the position of the KMTs. The polygon shape was calculated with the alpha shape algorithm $(\alpha=10)$ using the "ashape3d" function of the alphashape3d 1.3.1 R library (Lafarge and Pateiro-Lopez, 2020). The alpha shape is the polygon shape formed around a given set of points (KMTs from a cross-section) created by carving space around those points with a circle of a radius defined as $\alpha$. The polygon shape was then built by drawing outline polygon lines between contact points on the fitted circle. In order to calculate the polygon area from the polygon shape of the k-fiber cross-section, a polygon prism was created by duplicating and shifting a polygon shape $1 \mu \mathrm{m}$ in the $X Y Z$ dimension. This creates a prism with a height of $1 \mu \mathrm{m}$. The volume of created 3D object (prism) was then calculated with alphashape3d 1.3.1 R library (Lafarge and Pateiro-Lopez, 2020) and from this, a polygon area could be calculated by dividing the prism volume $\left(V_{p p}\right)$ by prism high $\left(h_{p p}=1 \mu \mathrm{m}\right)$. The distribution of the k-fiber polygon area along the pole-to-kinetochore axis is given as a fitted polynomial line of local polynomial regression using the "loess" method and confidence intervals were calculated with the t-based approximation using the stat 4.0.3 R library (R Core Team, 2021).

\section{Density of KMTs in k-fibers}

Also, the density of KMTs in the k-fibers was calculated in segments of $500 \mathrm{~nm}$ length along the entire path of each fiber. To determine the percentage of KMTs that were enclosed in the k-fiber for each cross-section, the number of KMTs enclosed in the given k-fiber section and circular area were determined. The radius of the circular area was calculated for each k-fiber at the position of the KMTs attached to the kinetochores. The distribution of the k-fiber density along the pole-to-pole axis is given as a fitted polynomial line and a confidence interval calculated with the t-based approximation using the stat 4.0.3 R library (R Core Team, 2021). 
596 The neighborhood KMTs at a given k-fiber cross-section were selected by K-nearest neighbor estimation. The estimation was achieved by firstly calculating a distance matrix between all selected KMTs. Given a distance matrix, each KMT-KMT connection was ranked according to its distance. Finally, for each KMT in a k-fiber, neighboring KMTs were selected by picking KMTs with $\mathrm{k}=1$. For each $\mathrm{k}$-fiber, a mean KMT-KMT neighborhood distance and standard deviation were calculated.

602 Interaction of MTs

603 The interaction between MTs was calculated for every $20 \mathrm{~nm}$ segment along each MT. For 604 that, for each MT segment, a distance to neighboring MTs was calculated. The MT-MT 605 interaction was defined if any given MT segment were closer or at an interaction distance. The 606 interaction distance between MTs was defined as 25, 30, 35, 45, $50 \mathrm{~nm}$. For visualization, 607 each MT segment was labeled based on the number of interactions. The length of interaction 608 between MTs was calculated as a sum of $20 \mathrm{~nm}$ segments. The frequency plot of average MT 609 interaction length and average interaction per MT was given for each interaction distance.

610

\section{Custom-designed software for the visualization of 3D data}

612 To better understand the 3D organization of KMTs in k-fibers, a visualization platform was 613 developed using the WebGL library (rgl 0.106.8 R library; (Adler et al., 2021)). This platform

614 was implemented for the public. This novel tool will allow readers to choose data sets from 615 this publication and visualize spindles according to selected features in an interactive way. For 616 instance, users may choose to visualize the organization of k-fibers or KMTs, and select for 617 the analysis of MT-MT. For an analysis of KMTs, users can select the following features of 618 analysis: length distribution, minus end positioning, curvature, and KMT number at the 619 kinetochore along with others. For the MT-MT interaction analysis, users can select different 620 interaction distances. This platform is designed to continuously add 3D reconstructions of 621 spindles obtained from different systems and can be accessed as follows: 622 https://cfci.shinyapps.io/ASGA 3DViewer/. 


\section{Data availability}

624 Tomographic data has been uploaded to the TU Dresden Open Access Repository and

625 Archive system (OpARA) and is available as open access: http://doi.org/10.25532/OPARA$626 \quad \underline{128}$

627 The code used to perform quantitative analysis of MT organization in spindles has been 628 uploaded to the GitHub repository and is available as open access under the GPL v3.0 license:

629 https://github.com/RRobert92/ASGA

\section{Acknowledgments}

631 The authors would like to thank Dr. Tobias Fürstenhaupt (Electron Microscopy Facility at the 632 MPI-CBG, Dresden, Germany) for technical support. We are also grateful to Drs. Reza 633 Farhadifar, Stefanie Redemann, Alejandra Laguillo Diego and Isabelle Vernos for a critical 634 reading of the manuscript. Research in the Müller-Reichert laboratory is supported by funds 635 from the Deutsche Forschungsgemeinschaft (MU 1423/8-2). R.K. received funding from the 636 European Union's Horizon 2020 research and innovation program under the Marie 637 Skłodowska-Curie grant agreement No. 675737 (grant to T.M.R.). This work was supported 638 by the NSF-Simons Center for Mathematical and Statistical Analysis of Biology at Harvard 639 (award number \#1764269), and the Harvard Quantitative Biology Initiative.

640

\section{Declaration of interests}

642 The authors declare no competing financial interests. 


\section{References}

644 Adler D, Murdoch D, Nenadic O, Urbanek S, Chen M, Gebhardt A, Bolker B, Csardi G, 645 Srzelecki A, Senger A. 2021. RGL - 3D visualization device system for R using OpenGL.

646 Amos LA. 1989. Brain dynein crossbridges microtubules into bundles. J Cell Sci 93:19-28.

647 Anjur-Dietrich MI, Kelleher CP, Needleman DJ. 2021. Mechanical Mechanisms of 648 Chromosome Segregation. Cells 10:465. doi:10.3390/cells10020465

649 Begley MA, Solon AL, Davis EM, Sherrill MG, Ohi R, Elting MW. 2021. K-fiber bundles in the 650 mitotic spindle are mechanically reinforced by Kif15. Mol Biol Cell 32:2020.05.19.104661. doi:10.1091/mbc.E20-06-0426

652

Campbell S, Amin MA, Varma D, Bidone TC. 2019. Computational model demonstrates that Ndc80-associated proteins strengthen kinetochore-microtubule attachments in metaphase. Cytoskeleton 76:549-561. doi:10.1002/cm.21562

655

Cherry LM, Faulkner AJ, Grossberg LA, Balczon R. 1989. Kinetochore size variation in mammalian chromosomes: an image analysis study with evolutionary implications. J Cell Sci 92:281-289.

Conway W, Kiewisz R, Fabig G, Kelleher, Colm, Wu, Hai-Yin, Anjur-Dietrich, Maya, MüllerReichert T, Needleman DJ. 2021. Self-organization of kinetochore-fibers in human mitotic spindles. bioRxiv.

DeLuca JG, Gall WE, Ciferri C, Cimini D, Musacchio A, Salmon ED. 2006. Kinetochore Microtubule Dynamics and Attachment Stability Are Regulated by Hec1. Cell 127:969982. doi:10.1016/j.cell.2006.09.047

Drpic D, Almeida AC, Aguiar P, Renda F, Damas J, Lewin HA, Larkin DM, Khodjakov A, Maiato 665 H. 2018. Chromosome Segregation Is Biased by Kinetochore Size. Curr Biol 28:1344666 1356.e5. doi:10.1016/j.cub.2018.03.023

Dumont S, Mitchison TJ. 2009. Force and Length in the Mitotic Spindle. Curr Biol 19:749-761. doi:10.1016/j.cub.2009.07.028

Fabig G, Kiewisz R, Lindow N, Powers JA, Cota V, Quintanilla LJ, Brugués J, Prohaska S, Chu DS, Müller-Reichert T. 2020. Male meiotic spindle features that efficiently segregate paired and lagging chromosomes. elife 9. doi:10.7554/eLife.50988 
673

674

675

676

677

678

679

680

681

682

683

684

685

686

687

688

689

690

691

692

693

694

695

696

697

698

699

700

701

702

703

modulates neuronal migration. Mol Biol Cell 22:1561-1574. doi:10.1091/mbc.e10-110905

Farhadifar R, Yu C-H, Fabig G, Wu H-Y, Stein DB, Rockman M, Müller-Reichert T, Shelley MJ, Needleman DJ. 2020. Stoichiometric interactions explain spindle dynamics and scaling across 100 million years of nematode evolution. elife 9:1-26. doi:10.7554/eLife.55877

Flemming W. 1879. Beitrage zur Kenntniss der Zelle und ihrer Lebenserscheinungen. Arch für Mikroskopische Anat 16:302-436. doi:10.1007/BF02956386

Godek KM, Kabeche L, Compton DA. 2015. Regulation of kinetochore-microtubule attachments through homeostatic control during mitosis. Nat Rev Mol Cell Biol 16:57-64. doi:10.1038/nrm3916

Guizetti J, Schermelleh L, Mäntler J, Maar S, Poser I, Leonhardt H, Müller-Reichert T, Gerlich DW. 2011. Cortical Constriction During Abscission Involves Helices of ESCRT-IIIDependent Filaments. Science 331:1616-1620. doi:10.1126/science.1201847

Hoffman DP, Shtengel G, Xu CS, Campbell KR, Freeman M, Wang L, Milkie DE, Pasolli HA, lyer N, Bogovic JA, Stabley DR, Shirinifard A, Pang S, Peale D, Schaefer K, Pomp W, Chang C-L, Lippincott-Schwartz J, Kirchhausen T, Solecki DJ, Betzig E, Hess HF. 2020. Correlative three-dimensional super-resolution and block-face electron microscopy of whole vitreously frozen cells. Science 367:eaaz5357. doi:10.1126/science.aaz5357

Inoué S. 1953. Polarization optical studies of the mitotic spindle. Chromosoma 5:487-500. doi:10.1007/BF01271498

Inoué S, Salmon ED. 1995. Force Generation by Microtubule Assembly/Disassembly in Mitosis and Related Movements. Mol Biol Cell 6:1619-1640. doi:10.1091/mbc.6.12.1619

Kajtez J, Solomatina A, Novak M, Polak B, Vukušić K, Rüdiger J, Cojoc G, Milas A, Šumanovac Šestak I, Risteski P, Tavano F, Klemm AH, Roscioli E, Welburn J, Cimini D, Glunčić M, Pavin N, Tolić IM. 2016. Overlap microtubules link sister k-fibres and balance the forces on bi-oriented kinetochores. Nat Commun 7:10298. doi:10.1038/ncomms 10298

Khodjakov A, Cole RW, McEwen BF, Buttle KF, Rieder CL. 1997. Chromosome Fragments Possessing Only One Kinetochore Can Congress to the Spindle Equator. J Cell Biol 136:229-240. doi:10.1083/jcb.136.2.229 
Kiewisz R, Müller-Reichert T. 2021. Automatic Spatial-Graph Analysis (ASGA). doi:https://doi.org/10.5281/zenodo.3732108

Kiewisz R, Müller-Reichert T, Fabig G. 2021. High-throughput screening of mitotic mammalian cells for electron microscopy using classic histological dyes In: Müller-Reichert T, Verkade P, editors. Methods in Cell Biology:Correlative Light and Electron Microscopy IV. New Jersey: Academic Press Inc. pp. 151-170. doi:10.1016/bs.mcb.2020.08.005

Kremer JR, Mastronarde DN, Mclntosh JR. 1996. Computer Visualization of ThreeDimensional Image Data Using IMOD. J Struct Biol 116:71-76. doi:10.1006/jsbi.1996.0013

Kuhn J, Dumont S. 2019. Mammalian kinetochores count attached microtubules in a sensitive and switch-like manner. J Cell Biol 218:3583-3596. doi:10.1083/jcb.201902105

Lafarge T, Pateiro-Lopez B. 2020. alphashape3d: Implementation of the 3D Alpha-Shape for the Reconstruction of 3D Sets from a Point Cloud.

Lindow N, Brünig FN, Dercksen VJ, Fabig G, Kiewisz R, Redemann S, Müller-Reichert T, Prohaska S, Baum D. 2021. Semi-automatic stitching of filamentous structures in image stacks from serial-section electron tomography. $J$ Microsc 284:25-44. doi:10.1111/jmi.13039

Long AF, Suresh P, Dumont S. 2020. Individual kinetochore-fibers locally dissipate force to maintain robust mammalian spindle structure. $J$ Cell Biol 219:1-13. doi:10.1083/jcb.201911090

Maiato H, Rieder CL, Khodjakov A. 2004. Kinetochore-driven formation of kinetochore fibers contributes to spindle assembly during animal mitosis. J Cell Biol 167:831-840. doi:10.1083/jcb.200407090

Mastronarde DN. 2005. Automated electron microscope tomography using robust prediction of specimen movements. J Struct Biol 152:36-51. doi:10.1016/j.jsb.2005.07.007

Mastronarde DN. 2003. SerialEM: A Program for Automated Tilt Series Acquisition on Tecnai Microscopes Using Prediction of Specimen Position. Microsc Microanal 9:1182-1183. doi:10.1017/S1431927603445911

Mastronarde DN. 1997. Dual-Axis Tomography: An Approach with Alignment Methods That Preserve Resolution. J Struct Biol 120:343-352. doi:10.1006/jsbi.1997.3919

Mastronarde DN, Held SR. 2017. Automated tilt series alignment and tomographic 
reconstruction in IMOD. J Struct Biol 197:102-113. doi:10.1016/j.jsb.2016.07.011

736

737

738

739

740

741

742

743

744

745

746

747

748

749

750

751

752

753

754

755

756

757

758

759

760

761

762

763

764

Mastronarde DN, McDonald KL, Ding R, McIntosh JR. 1993. Interpolar spindle microtubules in PTK cells. J Cell Biol 123:1475-1489. doi:10.1083/jcb.123.6.1475

McDonald KL, O'Toole ET, Mastronarde DN, Mclntosh JR. 1992. Kinetochore microtubules in PTK cells. J Cell Biol 118:369-383. doi:10.1083/jcb.118.2.369

McEwen BF, Ding Y, Heagle AB. 1998. Relevance of kinetochore size and microtubulebinding capacity for stable chromosome attachment during mitosis in PtK1 cells. Chromosom Res 6:123-132. doi:10.1023/A:1009239013215

McEwen BF, Heagle AB, Cassels GO, Buttle KF, Rieder CL. 1997. Kinetochore Fiber Maturation in PtK1 Cells and Its Implications for the Mechanisms of Chromosome Congression and Anaphase Onset. $J$ Cell Biol 137:1567-1580. doi:10.1083/jcb.137.7.1567

McIntosh JR, O’Toole E, Zhudenkov K, Morphew M, Schwartz C, Ataullakhanov FI, Grishchuk EL. 2013. Conserved and divergent features of kinetochores and spindle microtubule ends from five species. J Cell Biol 200:459-474. doi:10.1083/jcb.201209154

Metzner R. 1894. Beitrage zur Granulalehre. I. Kern und kerntheilung. Arch Anat Physiol 309348.

Mitchison T, Kirschner M. 1984. Dynamic instability of microtubule growth. Nature 312:237242. doi:10.1038/312237a0

Molodtsov MI, Mieck C, Dobbelaere J, Dammermann A, Westermann S, Vaziri A. 2016. A Force-Induced Directional Switch of a Molecular Motor Enables Parallel Microtubule Bundle Formation. Cell 167:539-552.e14. doi:10.1016/j.cell.2016.09.029

Monda JK, Cheeseman IM. 2018. The kinetochore-microtubule interface at a glance. J Cell Sci 131. doi:10.1242/jcs.214577

Muller-Reichert T, Hohenberg H, O'Toole ET, Mcdonald K. 2003. Cryoimmobilization and three-dimensional visualization of $C$. elegans ultrastructure. J Microsc 212:71-80. doi:10.1046/j.1365-2818.2003.01250.x

Musacchio A, Desai A. 2017. A Molecular View of Kinetochore Assembly and Function. Biology (Basel) 6:5. doi:10.3390/biology6010005

Nixon FM, Gutiérrez-Caballero C, Hood FE, Booth DG, Prior IA, Royle SJ. 2015. The mesh is 
a network of microtubule connectors that stabilizes individual kinetochore fibers of the mitotic spindle. elife 4:1-21. doi:10.7554/eLife.07635

767

768

769

770

771

772

773

774

775

776

777

778

779

780

781

782

783

784

785

786

787

788

789

790

791

792

793

794

795

Nixon FM, Honnor TR, Clarke NI, Starling GP, Beckett AJ, Johansen AM, Brettschneider JA, Prior IA, Royle SJ. 2017. Microtubule organization within mitotic spindles revealed by serial block face scanning EM and image analysis. J Cell Sci 130:1845-1855. doi:10.1242/jcs.203877

O'Toole E, Morphew M, McIntosh JR. 2020. Electron tomography reveals aspects of spindle structure important for mechanical stability at metaphase. Mol Biol Cell 31:184-195. doi:10.1091/mbc.E19-07-0405

O'Toole ET, McDonald KL, Mäntler J, Mclntosh JR, Hyman AA, Müller-Reichert T. 2003. Morphologically distinct microtubule ends in the mitotic centrosome of Caenorhabditis elegans. J Cell Biol 163:451-456. doi:10.1083/jcb.200304035

Oriola D, Needleman DJ, Brugués J. 2018. The Physics of the Metaphase Spindle. Annu Rev Biophys 47:655-673. doi:10.1146/annurev-biophys-060414-034107

Prosser SL, Pelletier L. 2017. Mitotic spindle assembly in animal cells: A fine balancing act. Nat Rev Mol Cell Biol. doi:10.1038/nrm.2016.162

R Core Team. 2021. R: A language and environment for statistical computing. R Foundation for Statistical Computing.

Redemann S, Baumgart J, Lindow N, Shelley M, Nazockdast E, Kratz A, Prohaska S, Brugués J, Fürthauer S, Müller-Reichert T. 2017. C. elegans chromosomes connect to centrosomes by anchoring into the spindle network. Nat Commun 8:15288. doi:10.1038/ncomms15288

Redemann S, Weber B, Möller M, Verbavatz J-M, Hyman AA, Baum D, Prohaska S, MüllerReichert T. 2014. The Segmentation of Microtubules in Electron Tomograms Using Amira In: Sharp DJ, editor. Mitosis: Methods and Protocols, Methods in Molecular Biology. New York: Springer Science+ Business Media. pp. 261-278. doi:10.1007/978-1-4939-03290_12

Reipert S, Fischer I, Wiche G. 2004. High-pressure freezing of epithelial cells on sapphire coverslips. J Microsc 213:81-85. doi:10.1111/j.1365-2818.2004.01260.x

Rieder CL. 1981. The structure of the cold-stable kinetochore fiber in metaphase PtK1 cells. Chromosoma 84:145-158. doi:10.1007/BF00293368 
Rieder CL, Salmon ED. 1998. The vertebrate cell kinetochore and its roles during mitosis. Trends Cell Biol 8:310.

Ris H, Witt PL. 1981. Structure of the mammalian kinetochore. Chromosoma 82:153-170. doi:10.1007/BF00286101

Rosselló C, Lindström L, Eklund G, Corvaisier M, Kristensson M. 2018. Y-Tubulin- - -Tubulin Interactions as the Basis for the Formation of a Meshwork. Int $J$ Mol Sci 19:3245. doi:10.3390/ijms 19103245

Schmidt WJ. 1939. Doppelbrechung der Kernspindel und Zugfasertheorie der Chromosomenbewegung. Chromosoma 1:253-264. doi:10.1007/BF01271634

Sikirzhytski V, Magidson V, Steinman JB, He J, Le Berre M, Tikhonenko I, Ault JG, McEwen BF, Chen JK, Sui H, Piel M, Kapoor TM, Khodjakov A. 2014. Direct kinetochore-spindle pole connections are not required for chromosome segregation. J Cell Biol 206:231-243. doi:10.1083/jcb.201401090

Sikirzhytski V, Renda F, Tikhonenko I, Magidson V, McEwen BF, Khodjakov A. 2018. Microtubules assemble near most kinetochores during early prometaphase in human cells. J Cell Biol 217:2647-2659. doi:10.1083/jcb.201710094

Stalling D, Westerhoff M, Hege H-C. 2005. Amira: a Highly Interactive System for Visual Data Analysis In: Hansen CD, Johnson CR, editors. The Visualization Handbook. Elsevier. pp. 749-767.

Tan R, Foster PJ, Needleman DJ, McKenney RJ. 2018. Cooperative Accumulation of DyneinDynactin at Microtubule Minus-Ends Drives Microtubule Network Reorganization. Dev Cell 44:233-247.e4. doi:10.1016/j.devcel.2017.12.023

Vukušić K, Buđa R, Bosilj A, Milas A, Pavin N, Tolić IM. 2017. Microtubule Sliding within the Bridging Fiber Pushes Kinetochore Fibers Apart to Segregate Chromosomes. Dev Cell 43:11-23.e6. doi:10.1016/j.devcel.2017.09.010

Weber B, Greenan G, Prohaska S, Baum D, Hege H-C, Müller-Reichert T, Hyman AA, Verbavatz J-M. 2012. Automated tracing of microtubules in electron tomograms of plastic embedded samples of Caenorhabditis elegans embryos. J Struct Biol 178:129-138. doi:10.1016/j.jsb.2011.12.004

Winey M, Mamay CL, O'Toole ET, Mastronarde DN, Giddings TH, McDonald KL, McIntosh JR. 1995. Three-dimensional ultrastructural analysis of the Saccharomyces cerevisiae 
bioRxiv preprint doi: https://doi.org/10.1101/2021.11.13.468347; this version posted November 13, 2021. The copyright holder for this preprint (which was not certified by peer review) is the author/funder. All rights reserved. No reuse allowed without permission.

828 

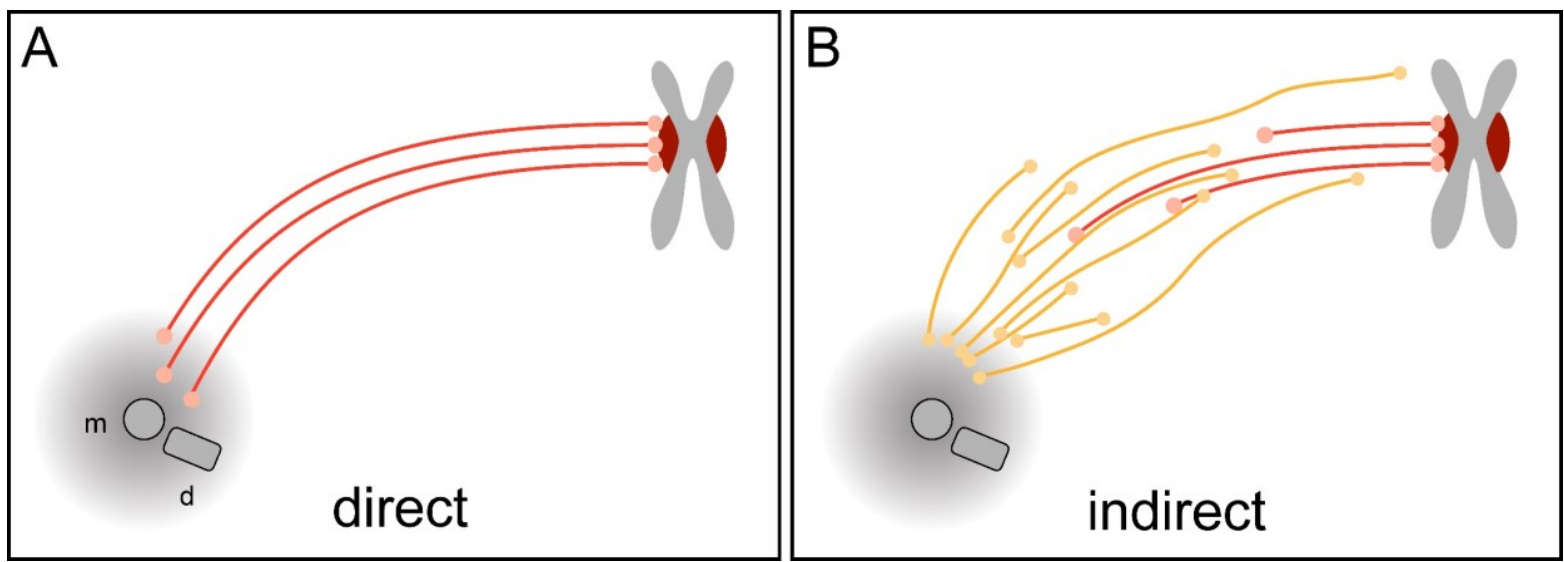

831 Figure 1. Proposed models on the organization of k-fibers in mammalian mitosis

833 (A) Direct connection with KMTs (red lines) spanning the distance between the kinetochore 834 and the spindle pole. The mother $(\mathrm{m})$ and the daughter centriole $(\mathrm{d})$ of the centrosome are 835 indicated. All KMTs are assumed to have the same length. (B) Indirect connection showing 836 KMTs linking the kinetochore and the centrosome by non-KMTs (yellow lines). K-fibers in this 837 model are composed of KMTs with different lengths, and none of the KMTs is directly 838 associated with the spindle pole. 

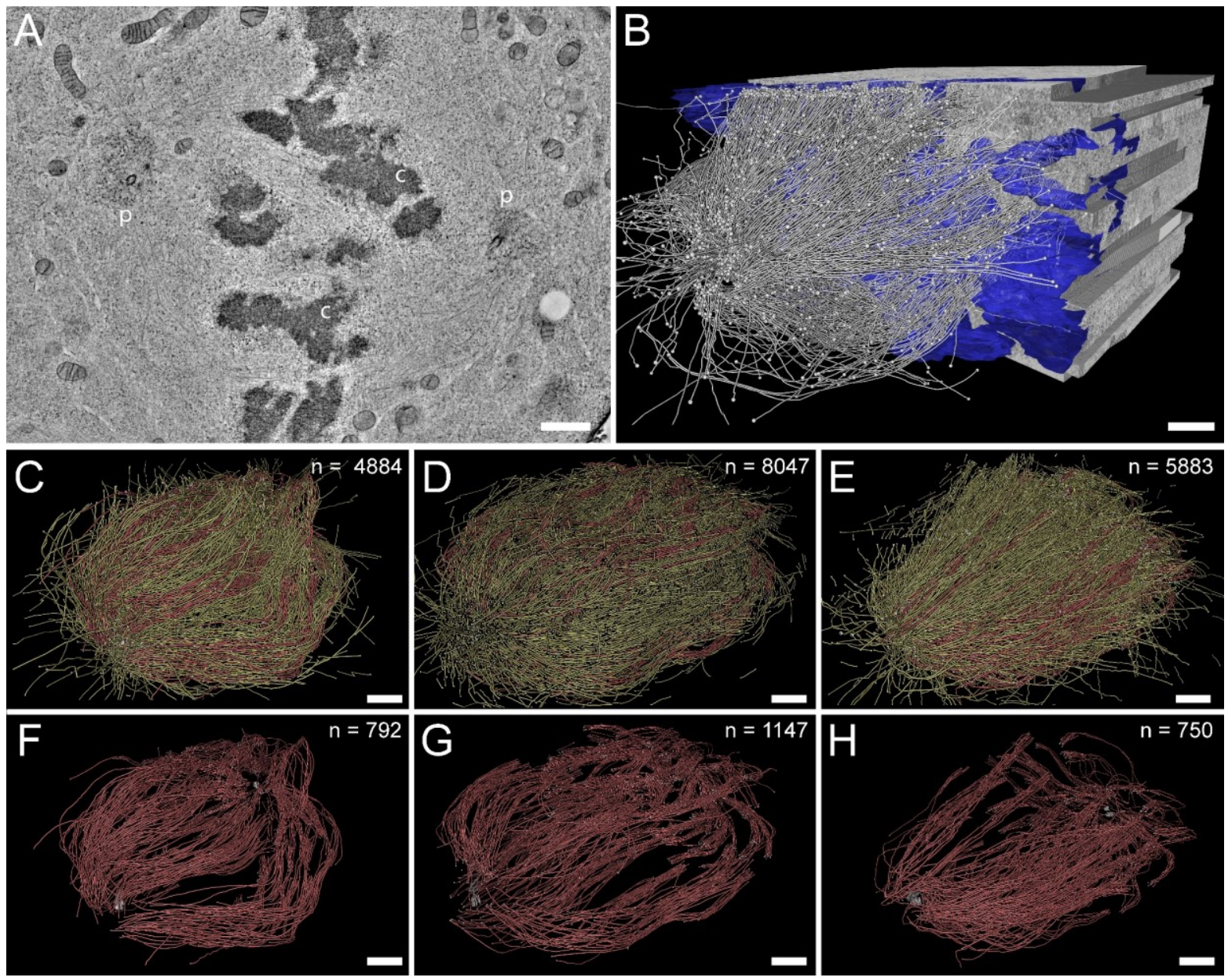

840 Figure 2. Three-dimensional reconstruction of metaphase spindles by large841 scale electron tomography

842 (A) Tomographic slice showing a HeLa cell (spindle \#1) in metaphase at low magnification.

843 The chromosomes (c) and the spindle poles ( $p$ ) are indicated. (B) Three-dimensional 844 reconstruction of the spindle in the same cell as shown in $A$. The stitching of the serial 845 tomograms to generate a three-dimensional model of the spindle with the MTs (white lines) is 846 illustrated. The segmented chromosomes are shown in blue. (C) Three-dimensional model of 847 the spindle as shown in A. The total number of microtubules is indicated (upper left corner). 848 The non-KMTs (yellow lines) and KMTs (red lines) are shown. (D) Full 3D model of metaphase 849 spindle \#2. (E) Full 3D model of metaphase spindle \#3. (F) Extraction of KMTs from the 3D 850 reconstruction as shown in $\mathrm{C}$. The number of KMTs for this spindle is indicated. (G) KMTs 851 extracted from spindle \#2. (H) KMTs extracted from spindle \#3. Scale bars, $1 \mu \mathrm{m}$. 

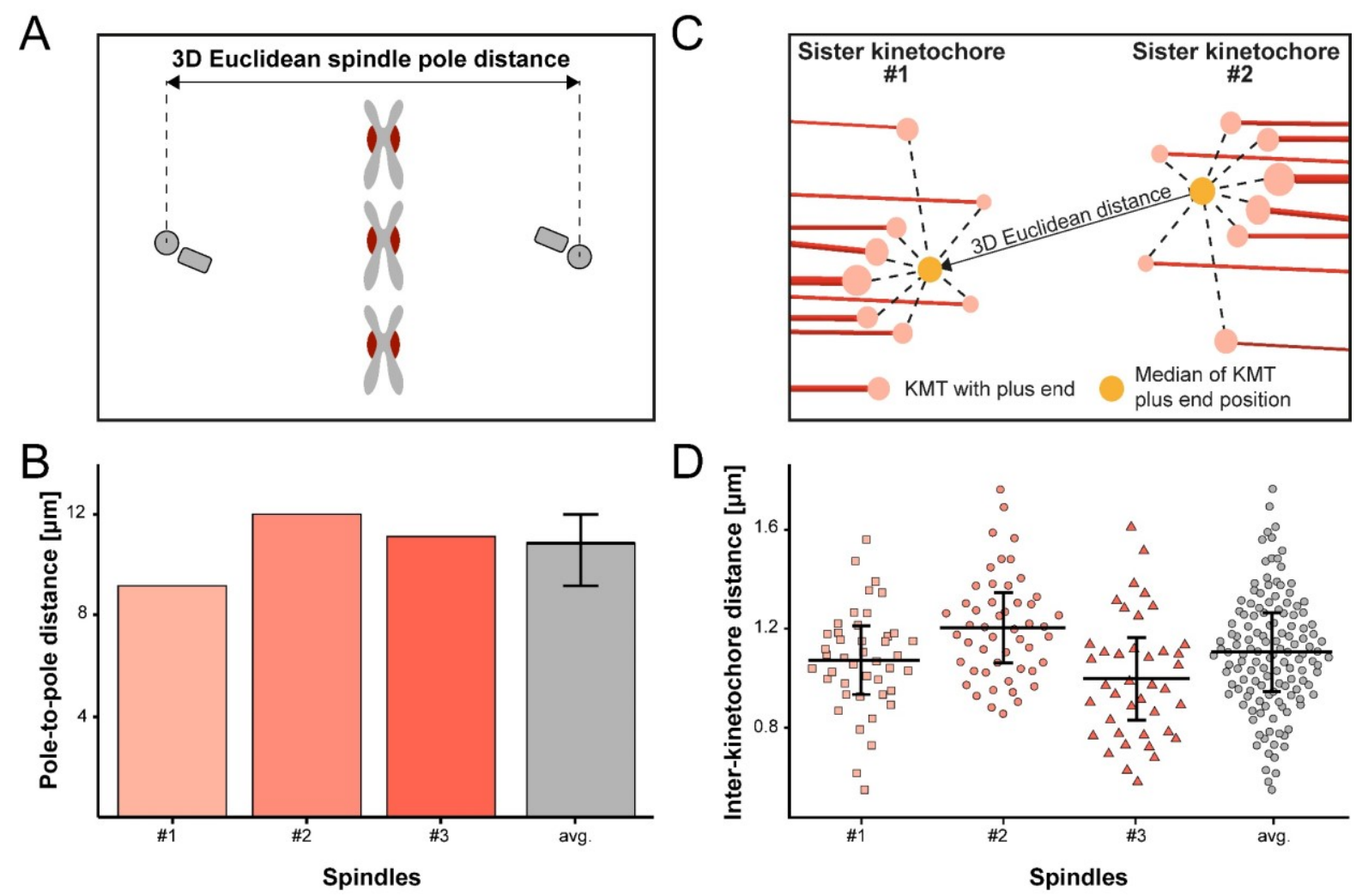

852

Figure 2. Three-dimensional reconstruction of metaphase spindles by largescale electron tomography

855 (A) Tomographic slice showing a HeLa cell (spindle \#1) in metaphase at low magnification.

856 The chromosomes (c) and the spindle poles (p) are indicated. (B) Three-dimensional 857 reconstruction of the spindle in the same cell as shown in $A$. The stitching of the serial 858 tomograms to generate a three-dimensional model of the spindle with the MTs (white lines) is 859 illustrated. The segmented chromosomes are shown in blue. (C) Three-dimensional model of 860 the spindle as shown in A. The total number of microtubules is indicated (upper left corner). 861 The non-KMTs (yellow lines) and KMTs (red lines) are shown. (D) Full 3D model of metaphase 862 spindle \#2. (E) Full 3D model of metaphase spindle \#3. (F) Extraction of KMTs from the 3D 863 reconstruction as shown in C. The number of KMTs for this spindle is indicated. (G) KMTs 864 extracted from spindle \#2. (H) KMTs extracted from spindle \#3. Scale bars, $1 \mu \mathrm{m}$. 

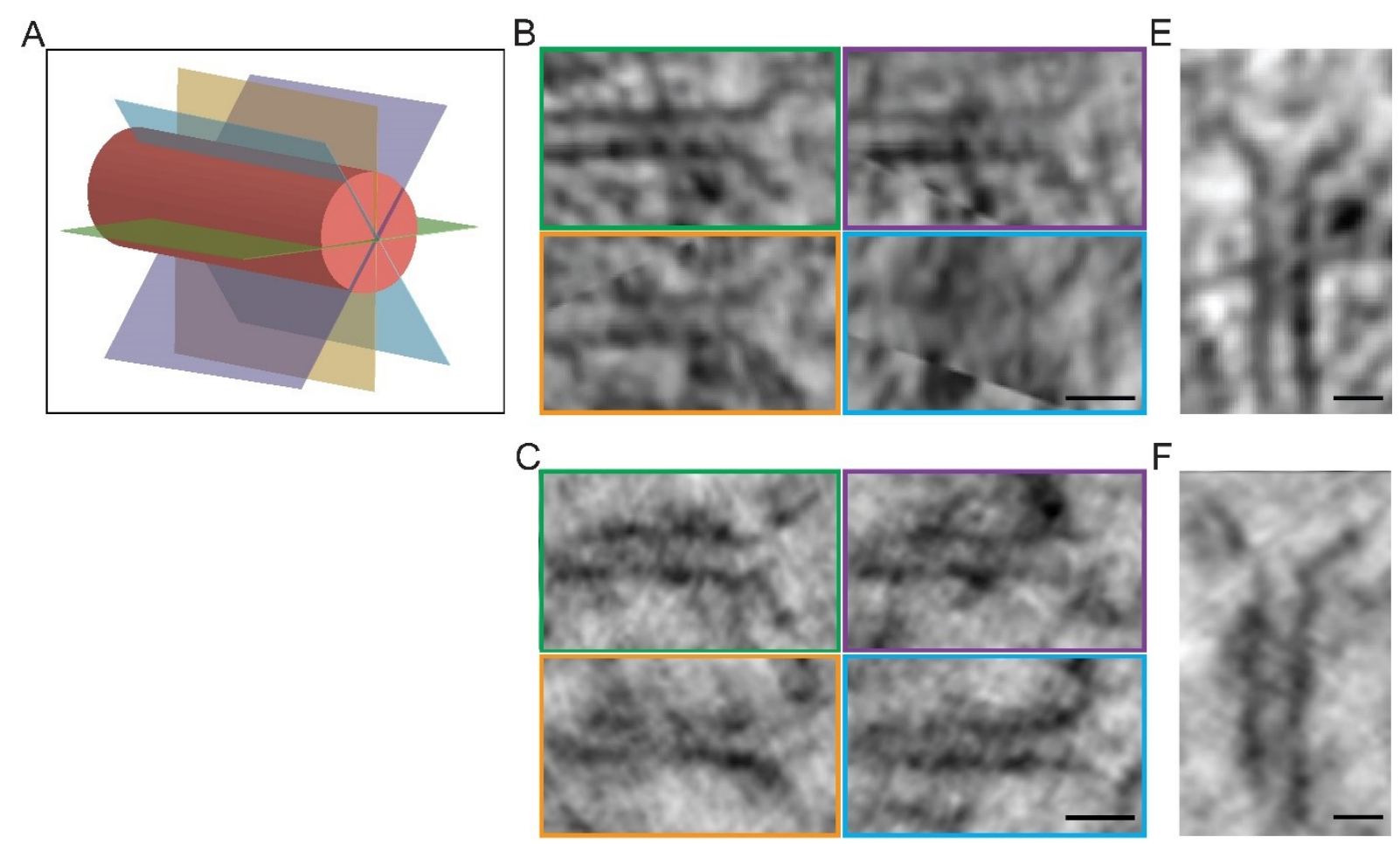

\section{$\mathrm{D}$}
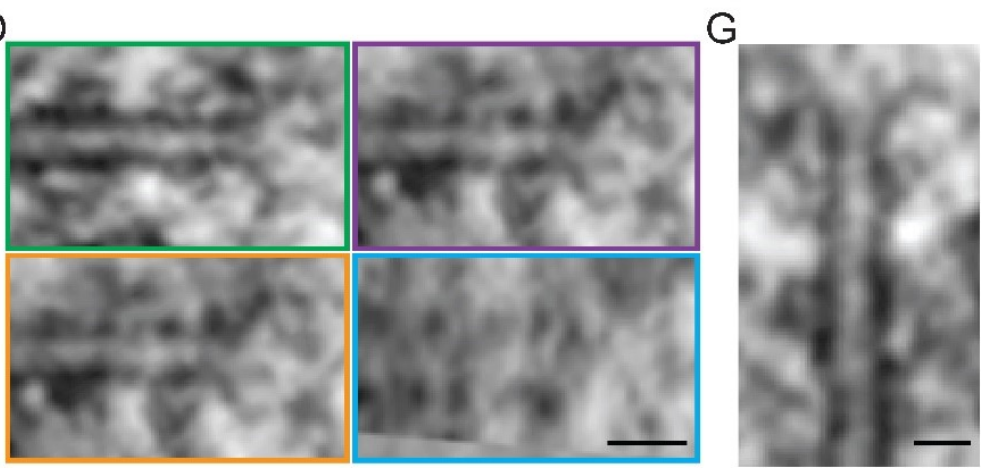

Figure 2-figure supplement 2. Selection of KMT plus ends based on end morphology

868 (A) Schematic illustration of MT cross-sections as used to visualize the end morphology of 869 KMTs. (B-D) Cross-sections of KMT plus ends as shown in A. (E-G) Average intensity z870 projection of KMT plus ends. 


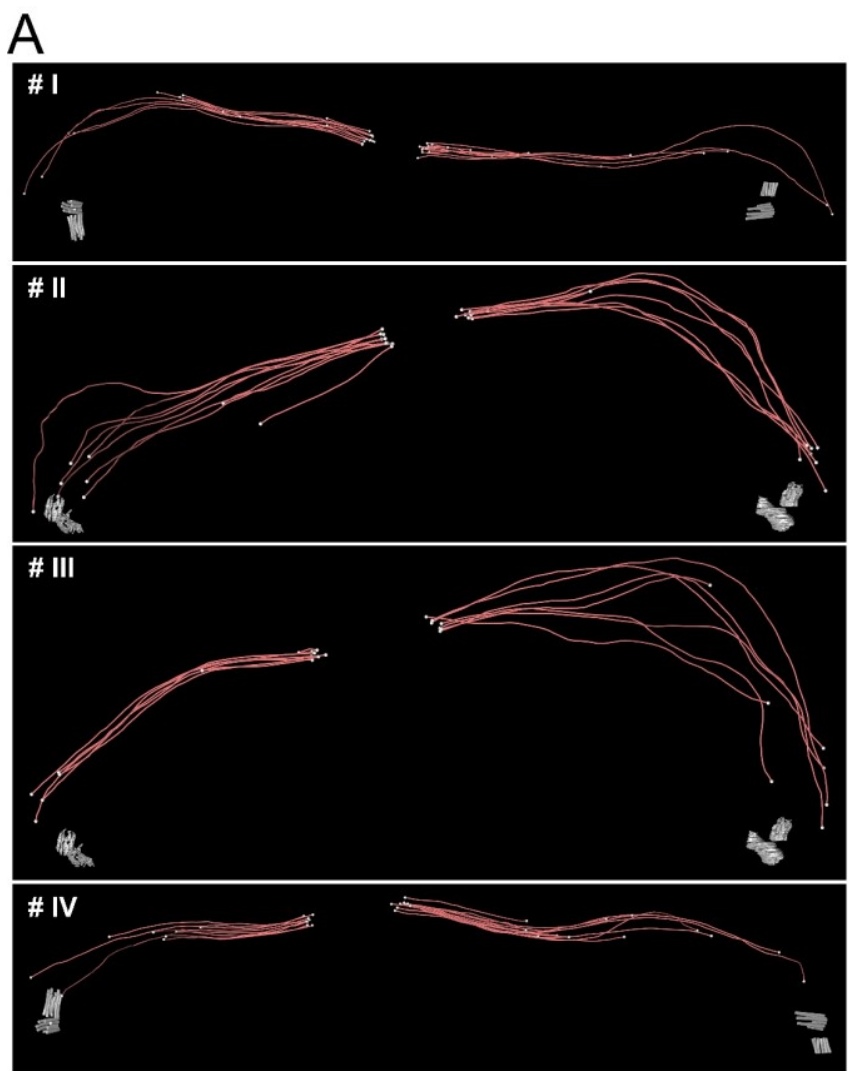

B
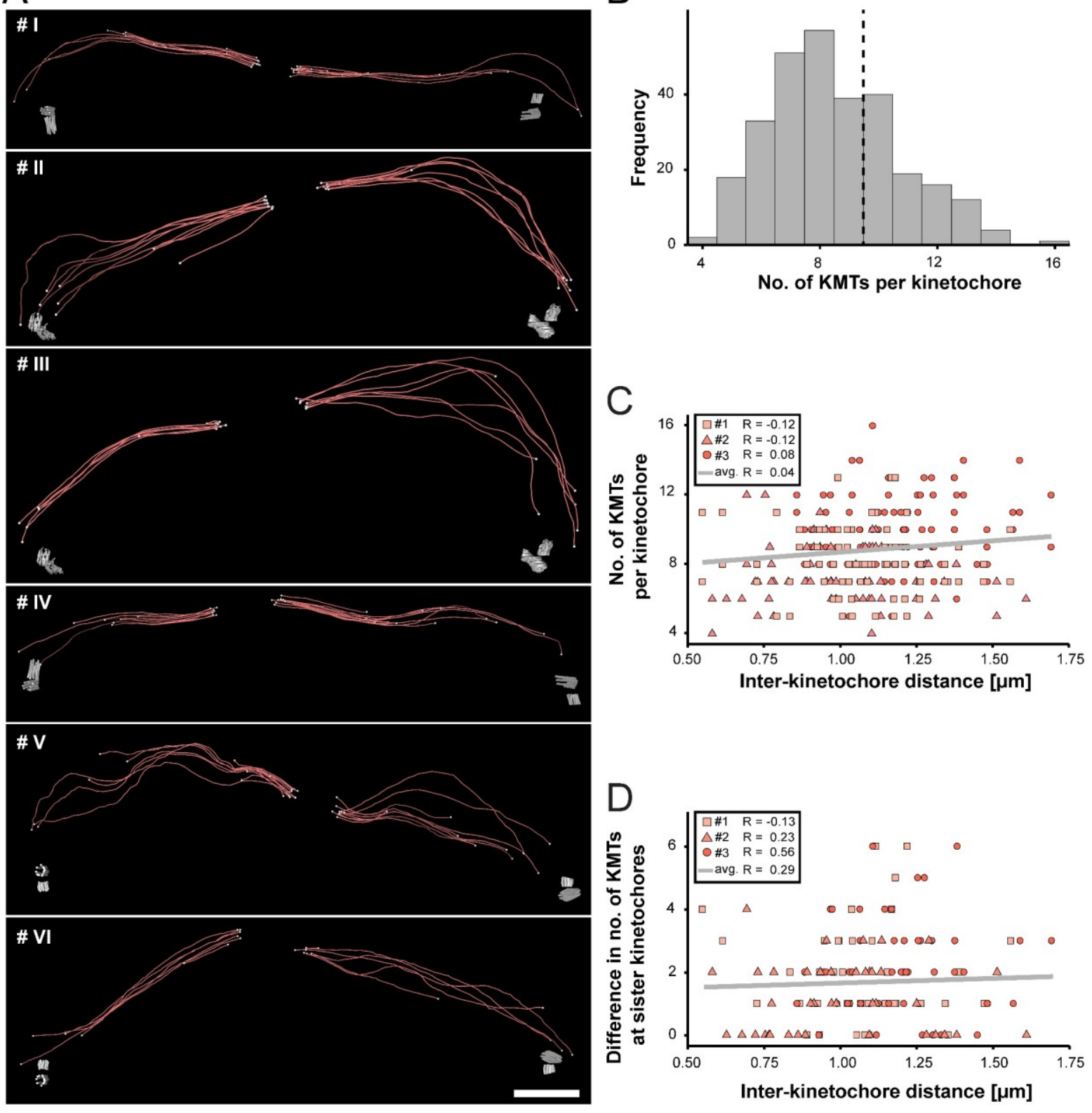

872 Figure 3. Number of KMTs attached per kinetochore

873 (A) Example models of individual sister k-fibers extracted from the full 3D reconstructions of 874 metaphase spindles. KMTs are shown as red lines. The ends of the KMTs are indicated by 875 white spheres. The centrioles (grey) are also shown. Scale bar, $1.5 \mu \mathrm{m}$. (B) Histogram plot 876 showing the frequency with which a given number of KMTs per kinetochore was detected. 877 This plot includes data from all three spindle reconstructions. (C) Correlation of the inter878 kinetochore distance and the number of KMTs associated per kinetochore. The Pearson's 879 correlation coefficient for each data set and the average for all data sets are given. (D) 880 Correlation of the inter-kinetochore distance and the difference (delta) in the number of KMTs 
bioRxiv preprint doi: https://doi.org/10.1101/2021.11.13.468347; this version posted November 13, 2021. The copyright holder for this preprint (which was not certified by peer review) is the author/funder. All rights reserved. No reuse allowed without permission.

881 associated with respective sister kinetochores. The Pearson's correlation coefficient for each 882 data set and the average for all data sets are given. 
A

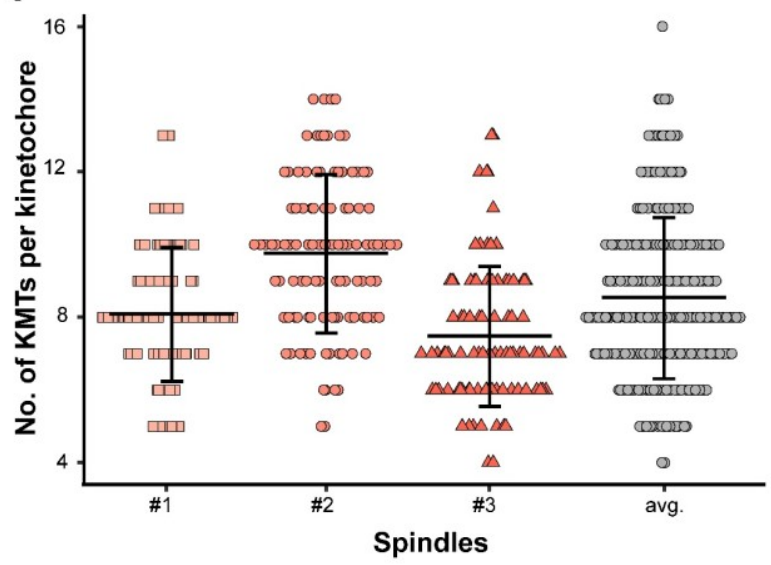

C

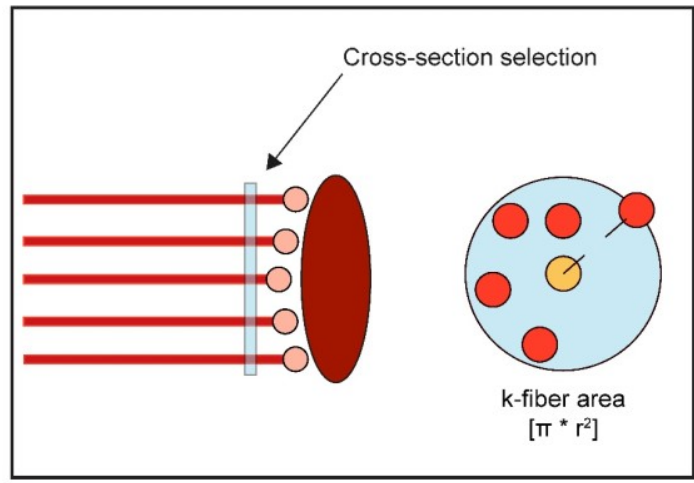

$\mathrm{E}$

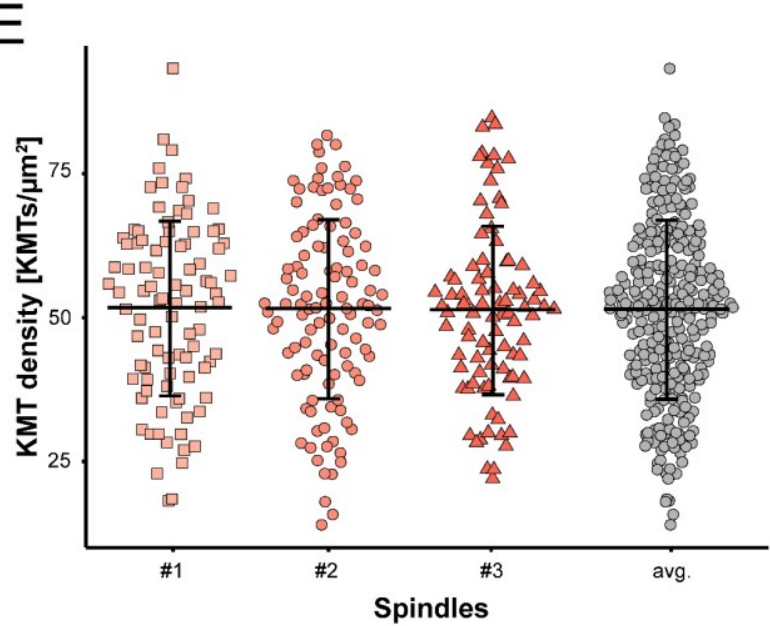

B

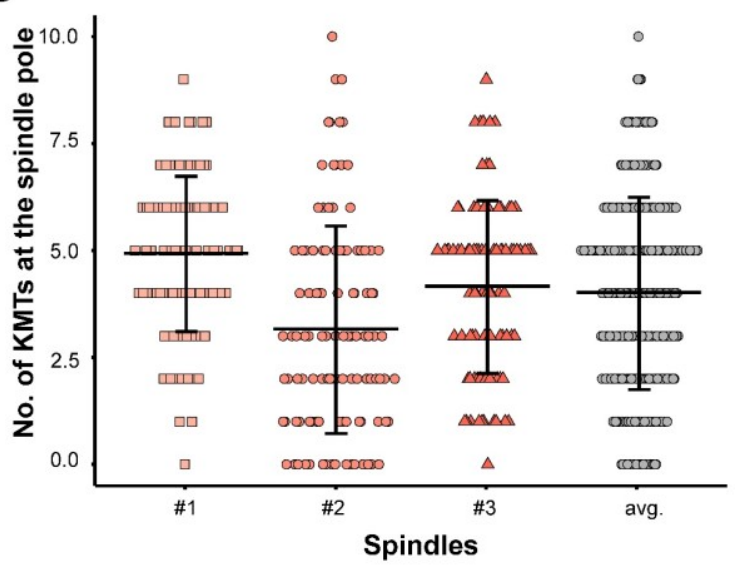

$\mathrm{D}$

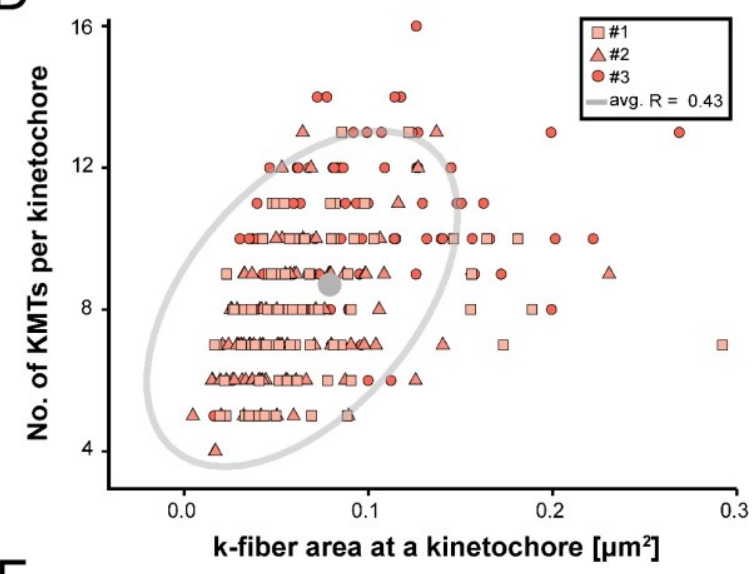

$\mathrm{F}$

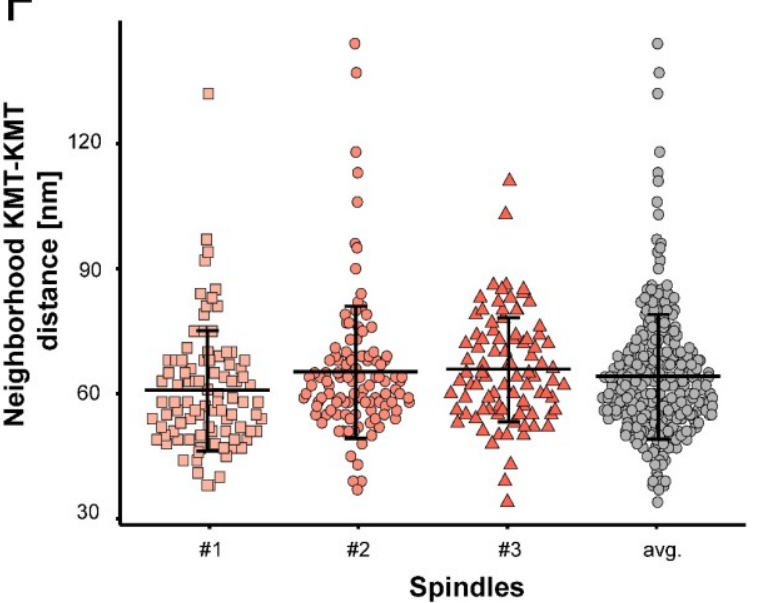

Figure 3-figure supplement 1 . Correlation of k-fiber circumference and number of attached KMTs

886 (A) Graph showing the number of KMTs per kinetochore for each data set and the average 887 number for all data sets (mean \pm STD). (B) Plot showing the distribution of KMT minus ends 888 in k-fibers for each data set and the average number for all data sets (mean \pm STD). (C) 889 Schematic illustration of the analysis of $k$-fiber area for indirect measurement of the 
890 kinetochore area. MTs (red lines) with their ends (spheres in light red) and the kinetochore 891 (ellipsoid in dark red) are shown. The measurement of the cross-section of a k-fiber in the 892 vicinity of the KMT ends is indicated by a blue bar (left). The k-fiber area is given by a circle 893 (blue) enclosing all KMTs (circle reaching to the middle of the peripheral KMTs). (D) 894 Correlation of k-fiber area and number of KMTs per kinetochore for all reconstructed spindles. 895 The grey ellipse indicates a 95\% confidence interval for all data and the grey dot indicates the 896 average kinetochore area. (E) Graph showing the density of KMTs at the kinetochore $897\left(\mathrm{KMTs} / \mu \mathrm{m}^{2}\right)$ for each 3D reconstruction and all data sets (mean $\left.\pm \mathrm{STD}\right)$. (F) Plot showing the 898 neighborhood KMT-KMT distance at the kinetochore for the individual data sets (mean \pm STD); 899 The average number for all data sets is also given. 
A

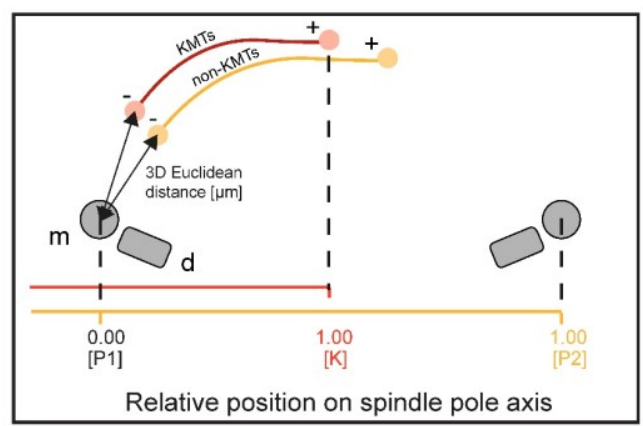

Relative position on spindle pole axis
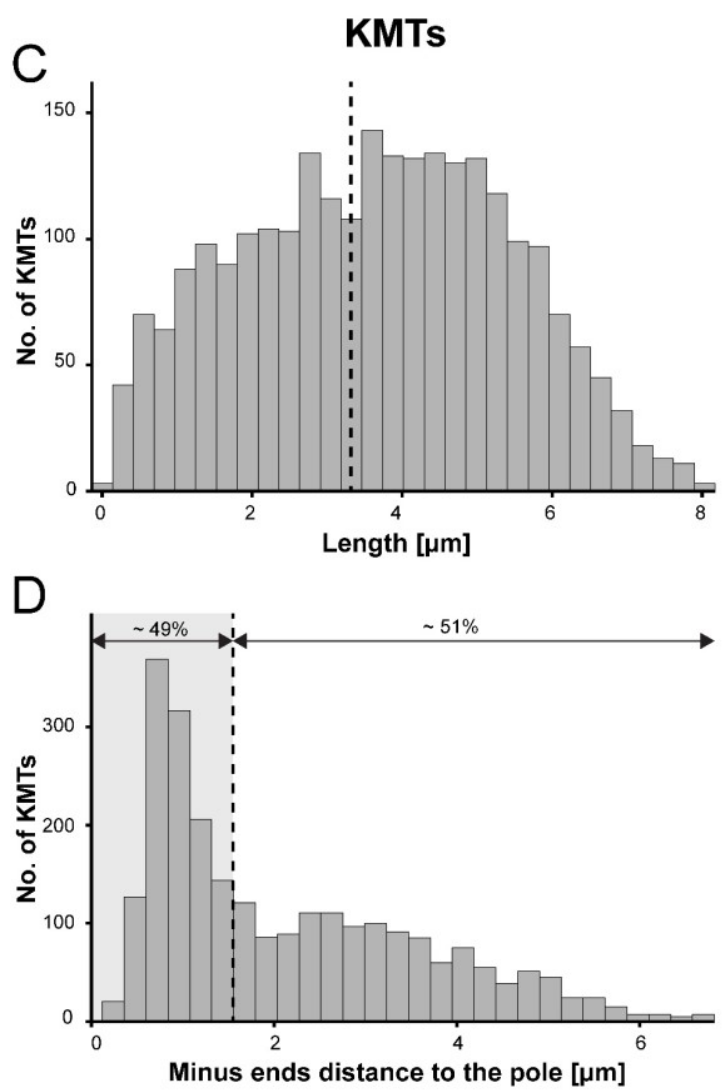

$E$

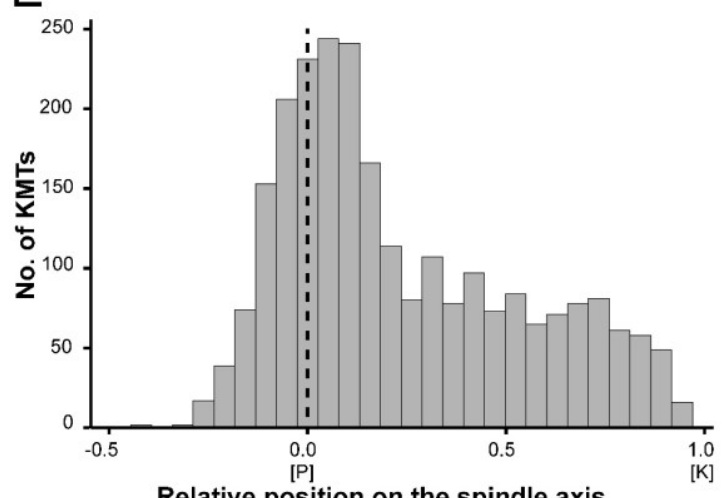

B

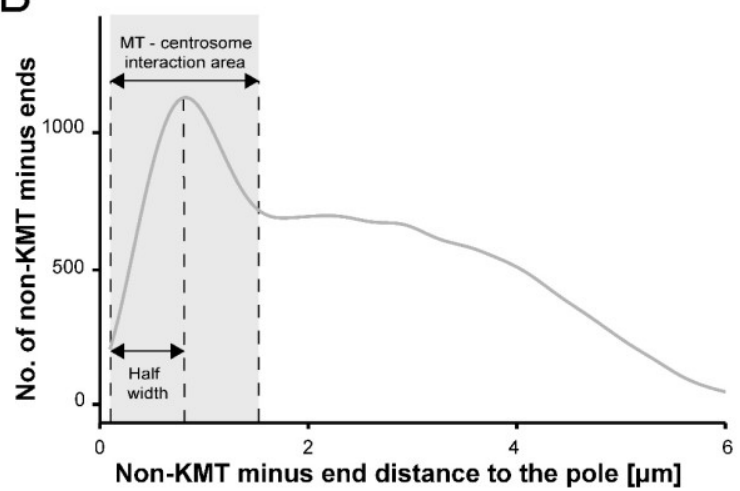

F

non-KMTs

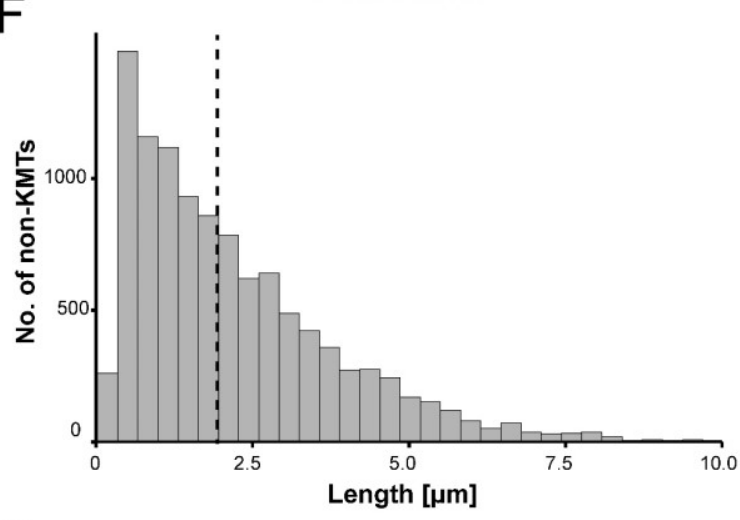

G

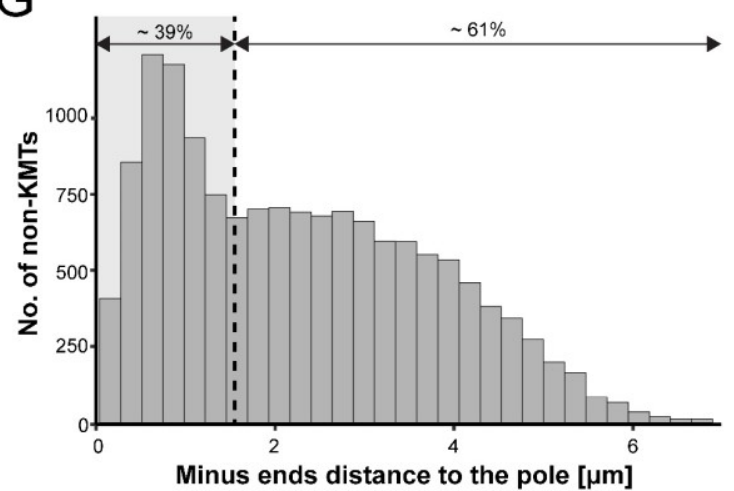

$\mathrm{H}$

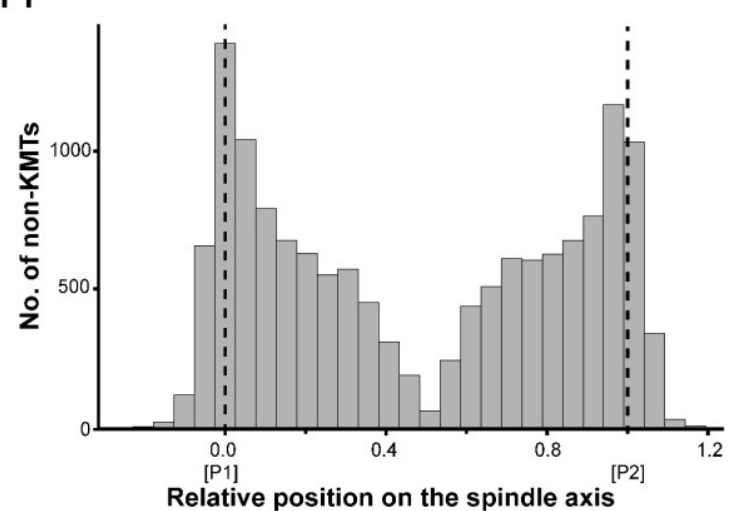




\section{Figure 4. Length distribution and minus end positioning of MTs in 902 metaphase spindles}

903 (A) Schematic illustration of MT minus end positioning along the spindle axis (P1 - pole1; P2 904 - pole2; K - kinetochore). A KMT (red line) with its ends (red spheres) and a non-KMT (yellow 905 line) with its ends (yellow spheres) are shown. The 3D Euclidean distance of both the KMT 906 and the non-KMT minus end to the center of the mother centriole can be calculated. The 907 relative position of the KMT minus ends along the pole-to-kinetochore axis and the non-KMT 908 minus ends along the pole-to-pole axis was also determined. (B) Calculation of the interaction 909 area of MTs with the centrosomes. Graph showing the number of non-KMT minus ends plotted 910 against their distance to the pole (i.e. to the center of the mother centriole). The determined 911 area of the interaction of non-KMTs with the centrosome and the half-width of this area is 912 indicated in grey. (C) Histogram showing the length distribution of KMTs from all data sets.

913 The dashed line indicates the average length of KMTs. (D) Histogram showing the distribution 914 of the KMT minus end distances to the center of the mother centriole. The area of interaction 915 of MTs with the centrosome is defined as in B and indicated by a grey area (dashed line shows 916 the border of this area). (E) Histogram showing the relative position of the KMT minus ends 917 on the spindle axis. The positions of the spindle pole ( $P=0$, dashed line) and the kinetochore $918(\mathrm{~K}=1)$ are indicated. (F) Histogram showing the length distribution of non-KMTs. The dashed 919 line indicates the average length of non-KMTs. (G) Histogram showing the distribution of the 920 non-KMT minus end distances to the center of the mother centriole. (H) Plot showing the 921 relative position of the non-KMT minus ends on the spindle axis. The positions of the spindle poles $(P 1=0, P 2=1)$ are indicated. 

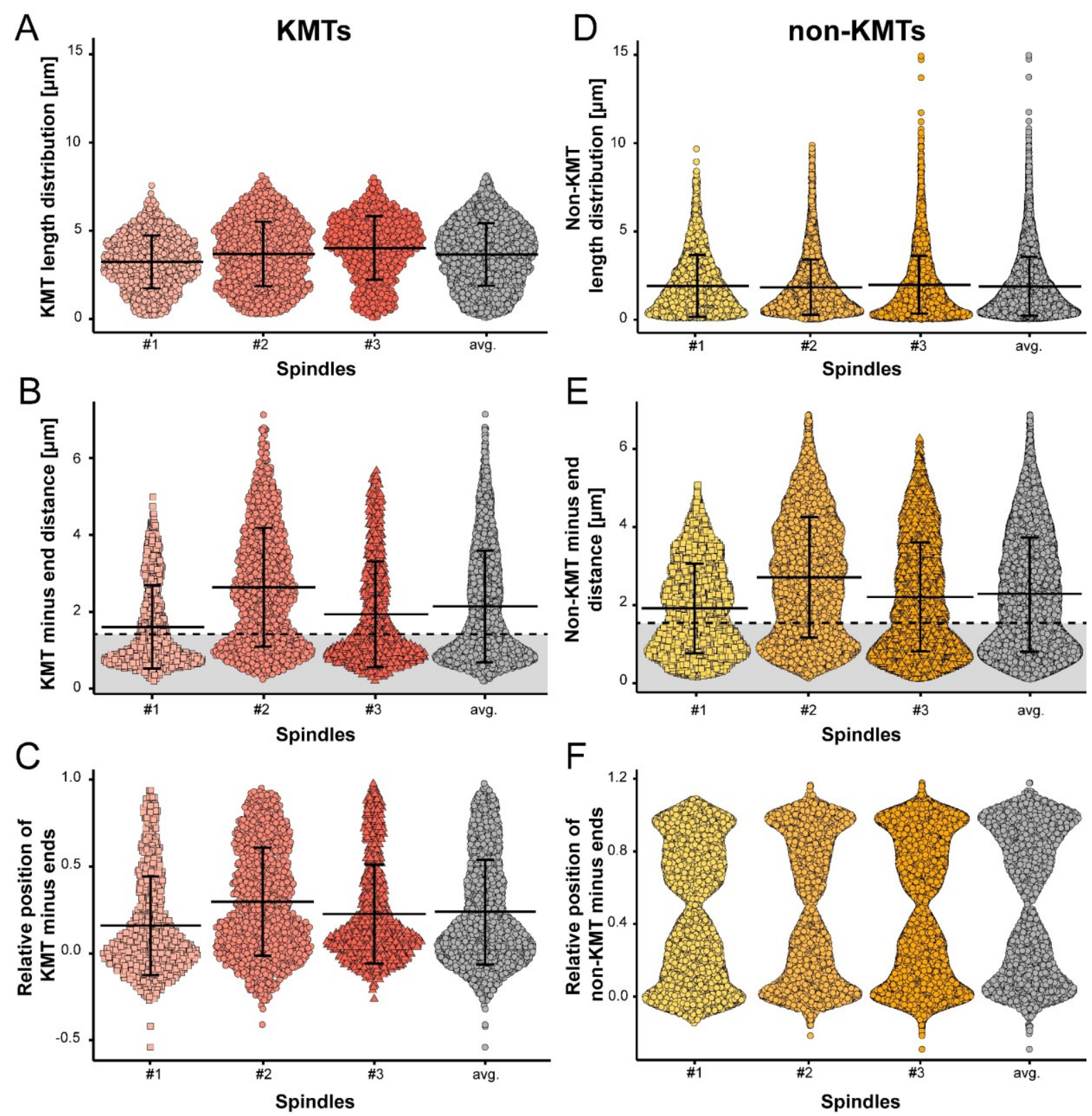

\section{Figure 4-figure supplement 1. KMT length and minus-end distribution}

925 (A) Beeswarm plot showing the length distribution of KMTs in all data sets and the average values. (B) Plot showing the distribution of KMT minus end distances to the center of the mother centriole. The area of interaction of the MT ends with the centrosomes is indicated in grey (with a dashed line showing the border of this area). (C) Plot showing the relative position of KMT minus ends on the spindle axis $(P=0, K=1)$.

(D) Length distribution of non-KMTs.

930 Distribution of the distances of the non-KMT minus ends to the center of the mother centriole.

931 (F) The relative position of non-KMT minus ends on the pole (P1)-to-pole (P2) axis. 

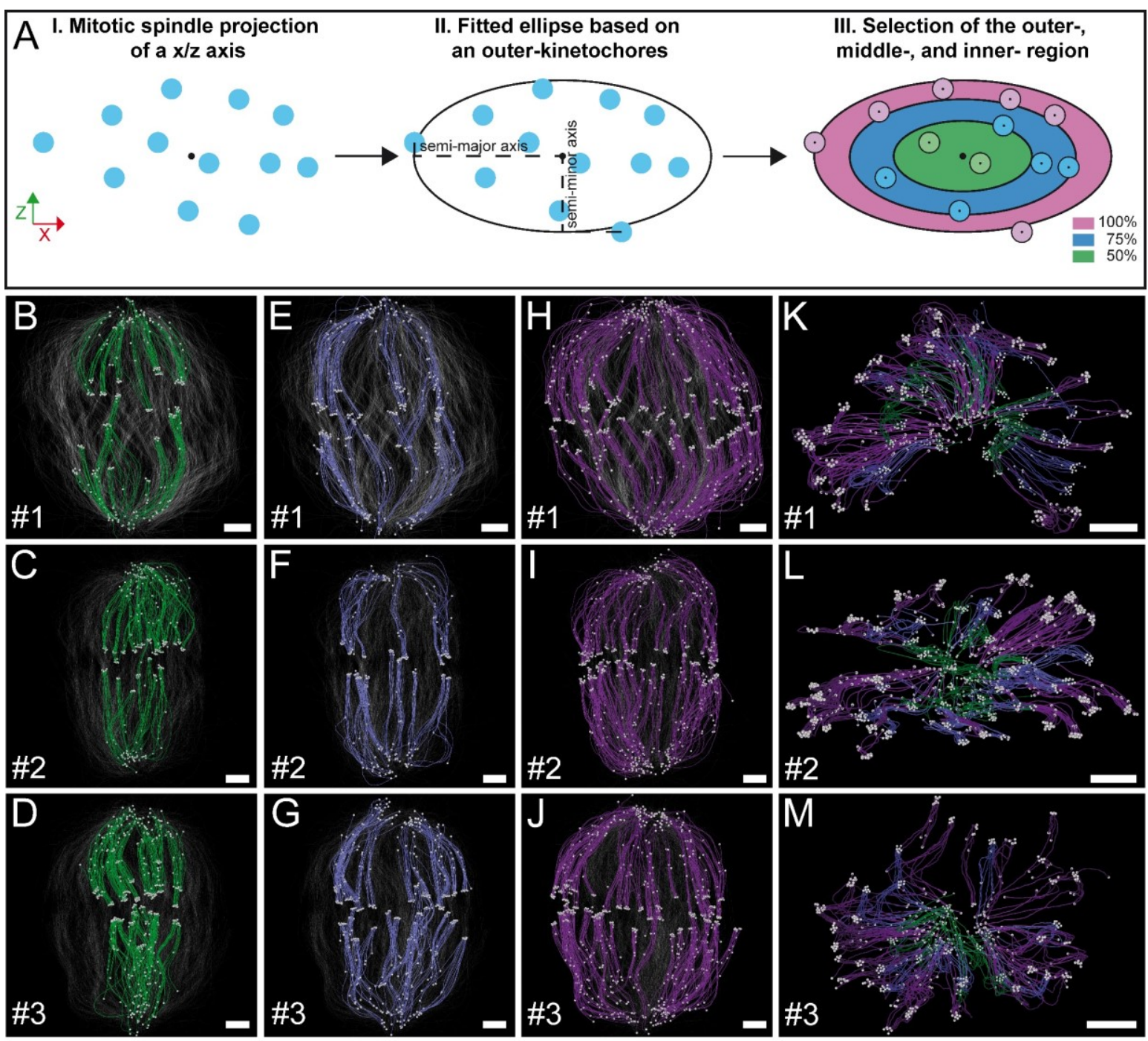

$\mathrm{N}$

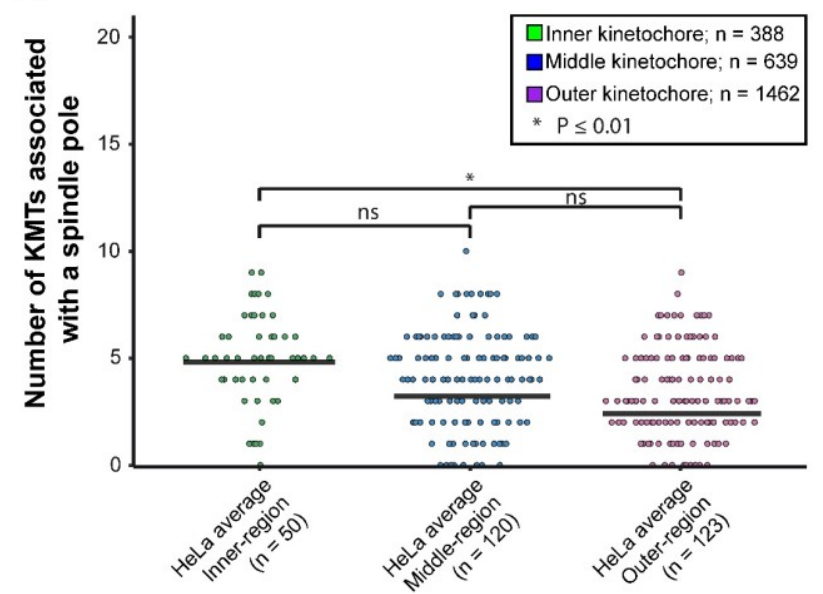

Figure 4-figure supplement 2. Correlation of KMT length and minus-end distribution with positioning on the metaphase plate

935 (A) Schematic illustration of the selection of kinetochores and the corresponding k-fiber 936 positions on the $x / z$ projection of the metaphase plate. Kinetochores are indicated as blue 
937 circles. The semi-minor and the semi-major axis indicate the axis of a fitted ellipse into all

938 kinetochores. The ellipse is divided into three parts representing the percentage of occupancy

939 of the ellipse (0-50\%, inner-kinetochores, green; 50-75\%, middle-kinetochores, blue; and 75-

$940100 \%$, outer-kinetochores, purple). (B-D) Perspective view on a metaphase spindle with

941 selected inner-kinetochores. (E-G) Perspective view with selected middle-kinetochores. (H-J)

942 Perspective view with selected outer-kinetochores. (K-M) Perspective view on a cross-section

943 of the spindle with all defined k-fiber classes. (N) Plot showing the number of KMT minus ends

944 in each k-fiber associated with the spindle pole for three positional regions in the mitotic

945 spindle for all data sets. The mean of the KMT minus-end number is indicated. 

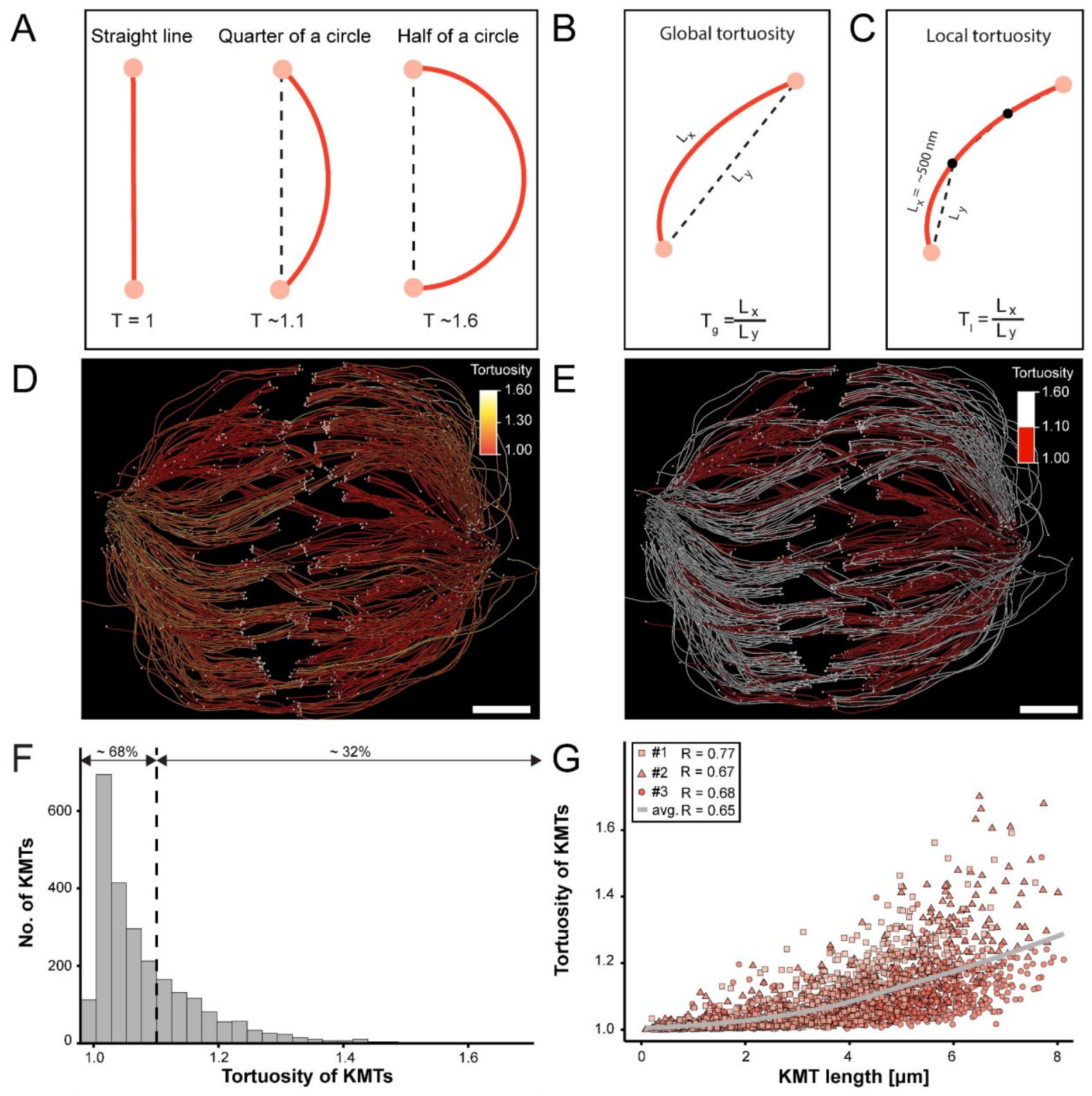

$\mathrm{H}$
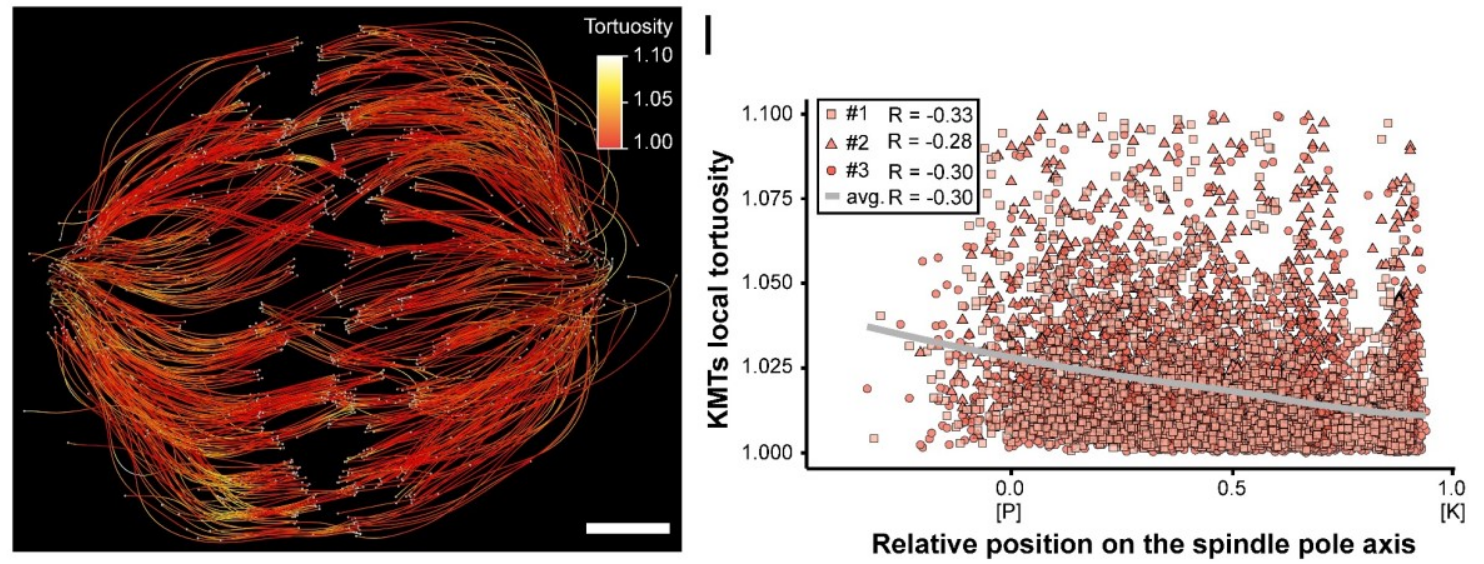


\section{Figure 5. Global and local tortuosity of KMTs}

948 (A) Schematic illustration of tortuosity $(T)$ as given for a straight line, and a quarter and a half 949 of a circle. (B) Schematic illustration of global tortuosity $\left(T_{g}\right)$ of KMTs given by the ratio of the 950 spline length $\left(L_{x}\right)$ to the 3D Euclidean distance between the KMT ends $\left(L_{y}\right)$. (C) Schematic 951 illustration of KMT local tortuosity $\left(T_{1}\right)$ as given by division segments with a length of $500 \mathrm{~nm}$.

952 (D) Three-dimensional model of k-fibers (spindle \#1) showing the global tortuosity of KMTs as 953 indicated by color coding (top left corner). (E) Perspective view as shown in C. Straight KMTs 954 (tortuosity of $1.0-1.1$, red) and curved KMTs (tortuosity $\geq 1.1$, white) are highlighted. (F) 955 Histogram showing the frequency of different degrees of KMT tortuosity. A tortuosity of 1.1 is 956 indicated by a dashed line. (G) Correlation of the global tortuosity and the length of KMTs. The 957 Pearson's correlation coefficient is given for each reconstructed spindle. The grey line 958 indicates the local regression calculated by the loess method. (H) Three-dimensional model 959 of k-fibers (spindle \#1) showing the local tortuosity of KMTs as indicated by color-coding. (I) 960 Correlation of the local tortuosity of KMTs with the relative position along the pole (P)-to961 kinetochore $(\mathrm{K})$ distance. Scale bars, $1 \mu \mathrm{m}$. 

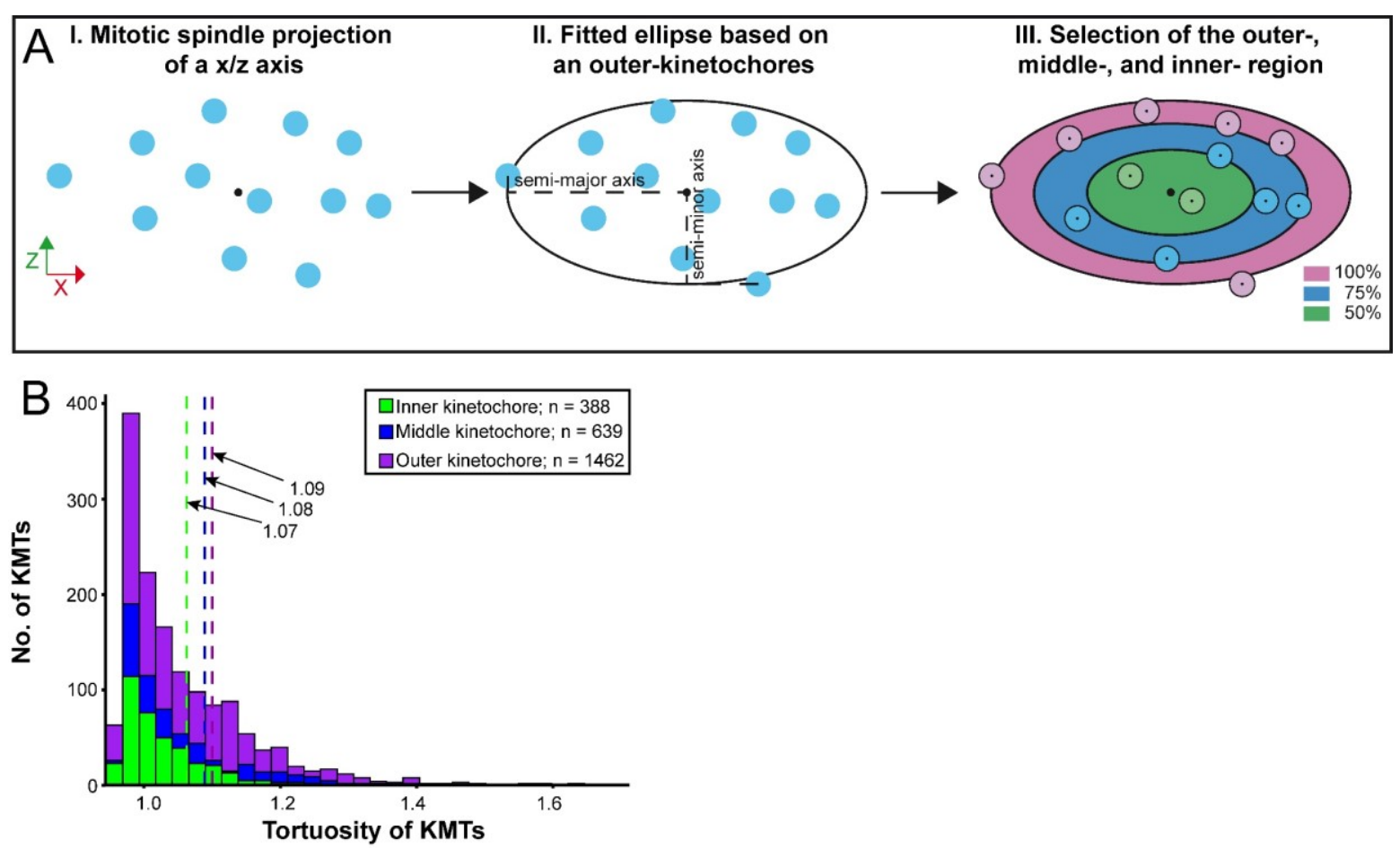

964 Figure 5-figure supplement 1. Correlation of MT tortuosity with k-fiber 965 positioning in the spindle

966 (A) Selection of kinetochore and corresponding k-fiber positions on the $x / z$ projection of the 967 metaphase plate. Kinetochores are indicated as blue circles. The semi-minor and the semi968 major axis indicate the axis of a fitted ellipse into all kinetochores. The ellipse is divided into 969 three parts representing the percentage of occupancy of the ellipse $(0-50 \%$, inner970 kinetochores, green; $50-75 \%$, middle-kinetochores, blue; and $75-100 \%$, outer-kinetochores, 971 purple). (B) Histogram showing the frequency of different degrees of KMT tortuosity in relation 972 to k-fiber position in the mitotic spindle. The mean tortuosity of each position class is indicated. 
A

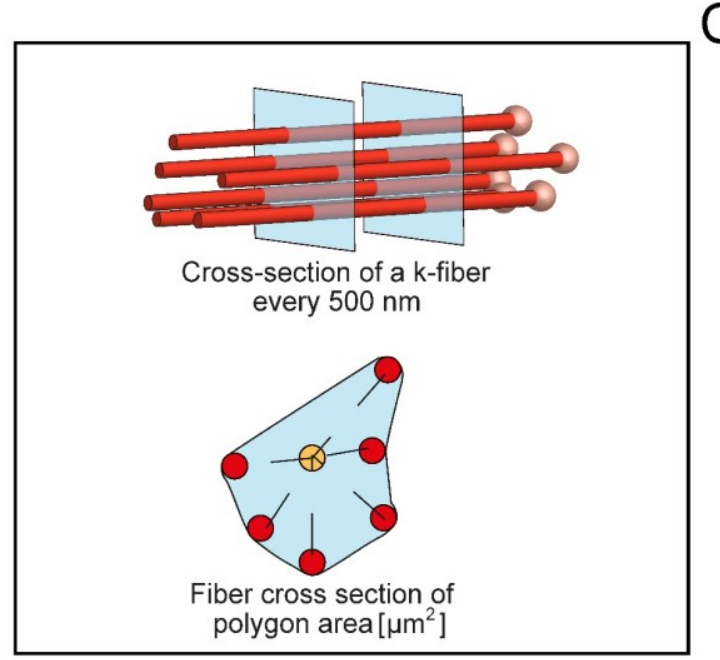

C
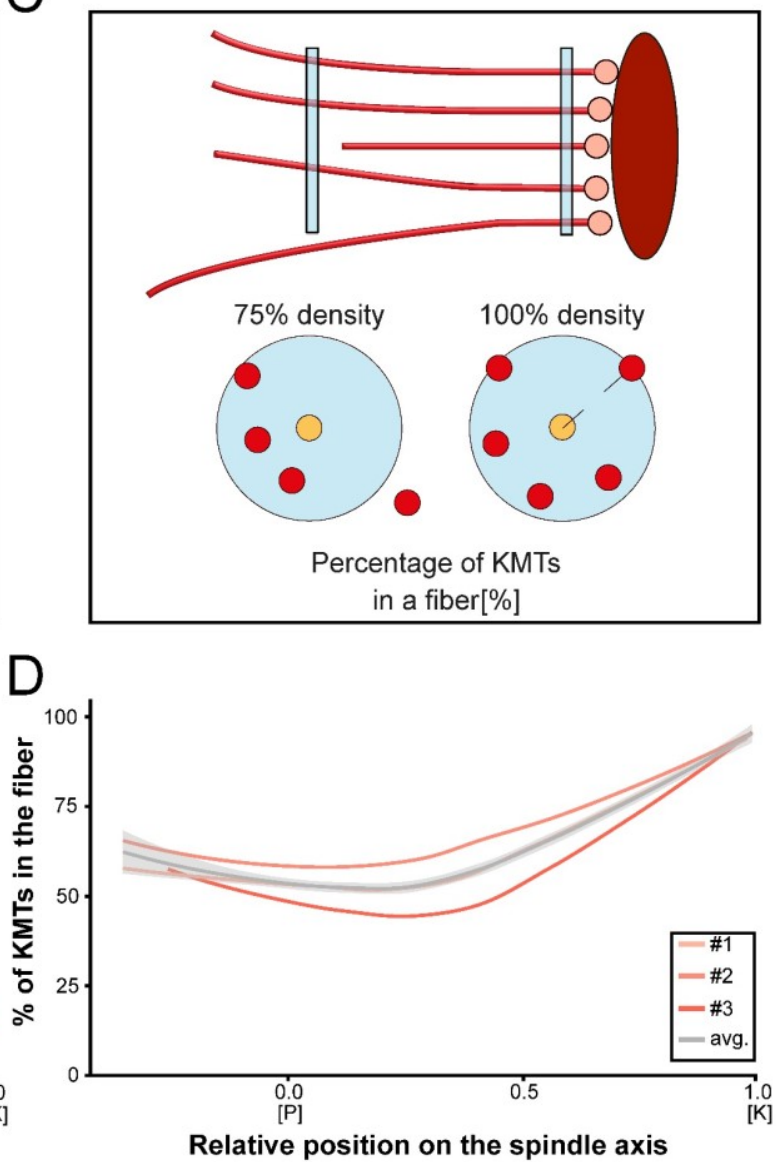

Relative position on the spindle axis

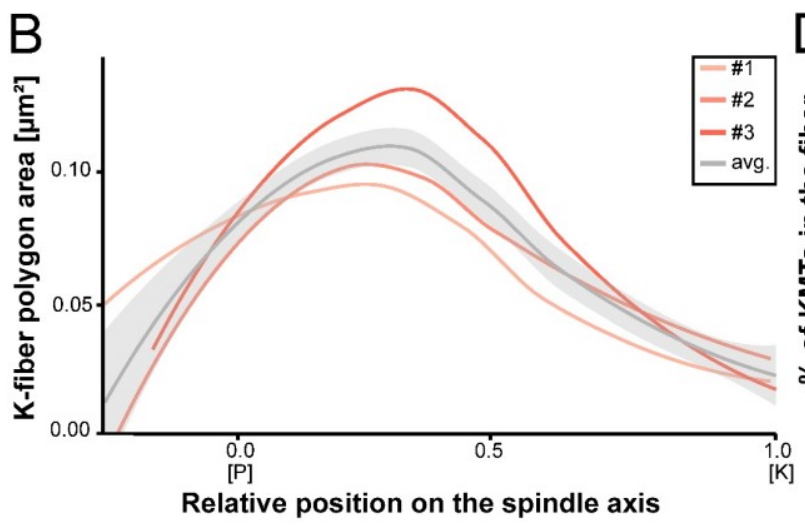

\section{$974 \quad$ Figure 6. Shape of k-fibers}

975 (A) Schematic illustration of the polygon area-analysis of k-fiber cross-sections. KMTs are

976 shown in red, KMT ends as red spheres. The cross-section of the given k-fiber is shown as a

977 blue square and the median position of all KMTs in the cross-section as a yellow circle. (B)

978 Correlation of the k-fiber polygon area and the relative position on the pole [P]-to-kinetochore

$979[\mathrm{~K}]$ axis. (C) Schematic illustration of the k-fiber density analysis. For each k-fiber, a radius at 980 the kinetochore was estimated by calculating a minimum circle enclosing all KMTs. The 981 determined radius was then enlarged by factor 2 to account for k-fiber flexibility. Along with 982 the k-fiber, the number of KMTs enclosed in the selected radius was then measured. (D) 983 Correlation of the KMT density and the relative position along pole [P]-to-kinetochore [K] axis.

984 For each reconstructed spindle, data sets are presented as polynomial lines showing local 985 regression calculated with the loess method. Average values with standard deviations are shown in grey. 


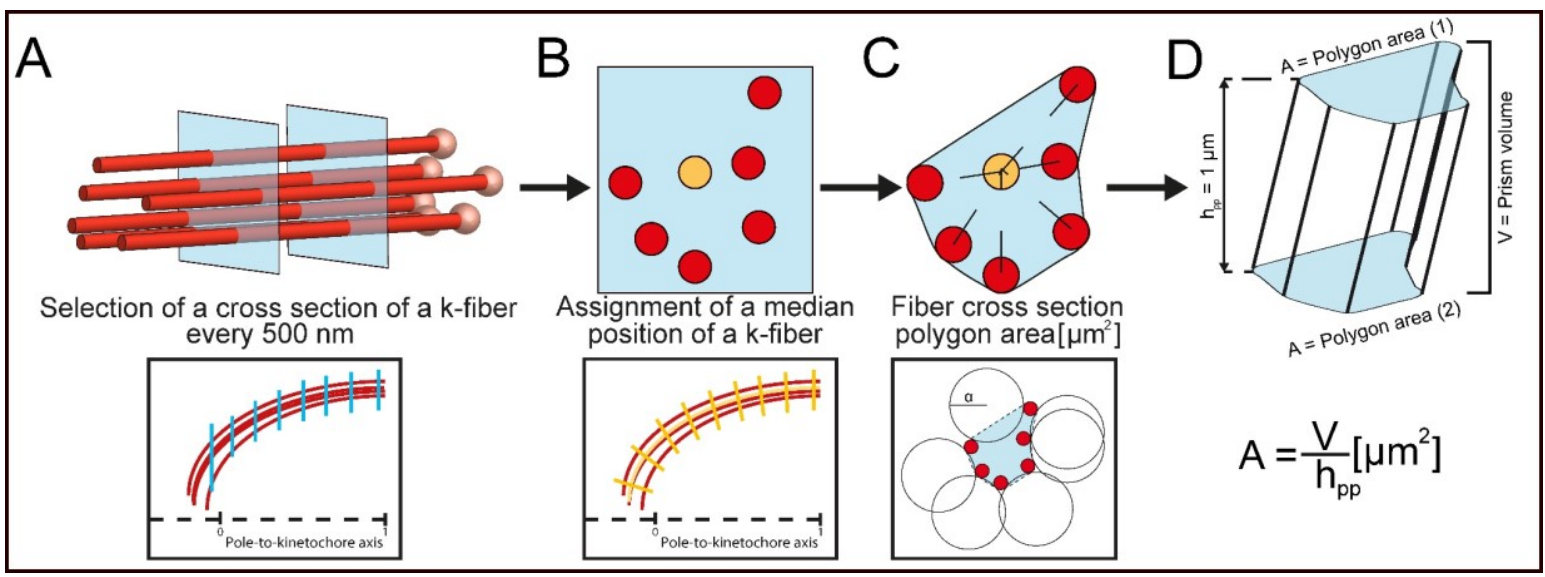

988 Figure 6-figure supplement 1. Schematic illustration of the analysis of kfiber area and density

990 (A) Selection of cross-sections on a k-fiber (KMTs in red, KMT ends, red spheres) every 500 $991 \mathrm{~nm}$ (upper row - blue squares; lower row - blue lines). (B) Assignment of a median position 992 (upper row - yellow circle) for each cross-section and geometric correction of each cross993 section based on the k-fiber's curvature (lower row - yellow lines). (C) Calculation of the 994 polygon area (upper row). The polygon area was calculated using the alpha shape algorithm. 995 For each cross-section, circles with diameter defined by a (lower row - black lines) were fitted 996 around the 2D projection of KMTs. Polygon shape was created by drawing lines around KMTs 997 that were at the junction of fitted circles (lower row - black dashed lines). (D) Calculation of the 998 polygon area from the created prism shape. For this, a polygon area was duplicated and 999 moved in XYZ Euclidean space by $1 \mu \mathrm{m}$ to create a prism shape. The polygon area $(\mathrm{A})$ is 1000 given by dividing a prism volume $\left[\mu \mathrm{m}^{2}\right]$ by a prism high $\left(\mathrm{h}_{\mathrm{pp}}=1 \mu \mathrm{m}\right)$. 


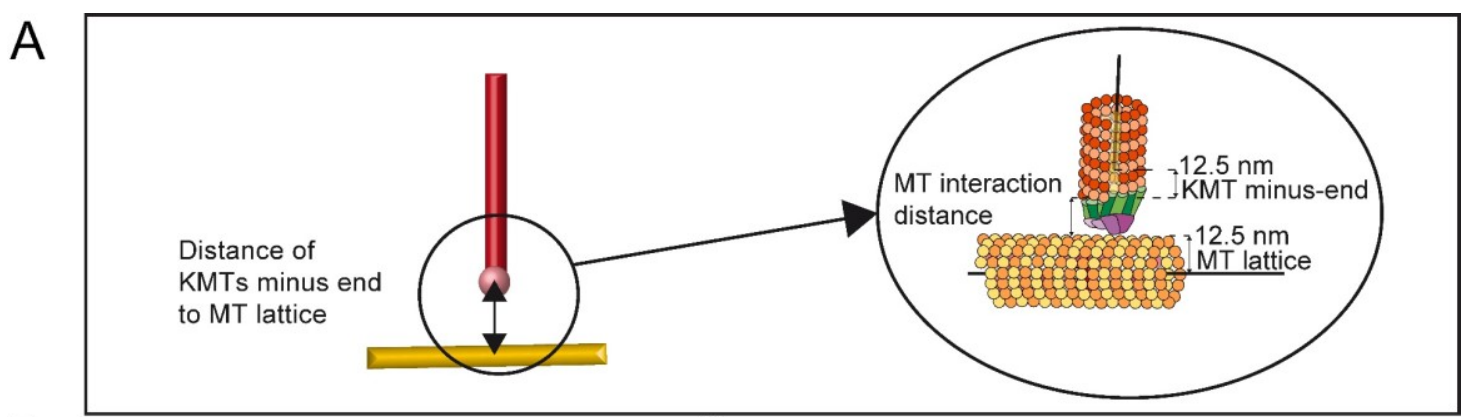

$\mathrm{B}$

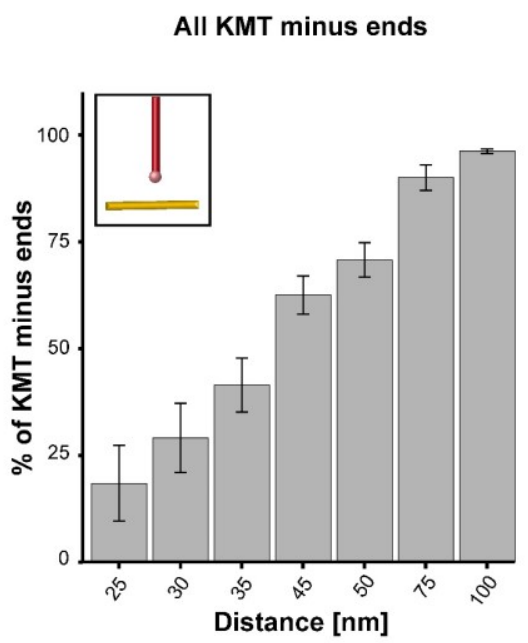

D

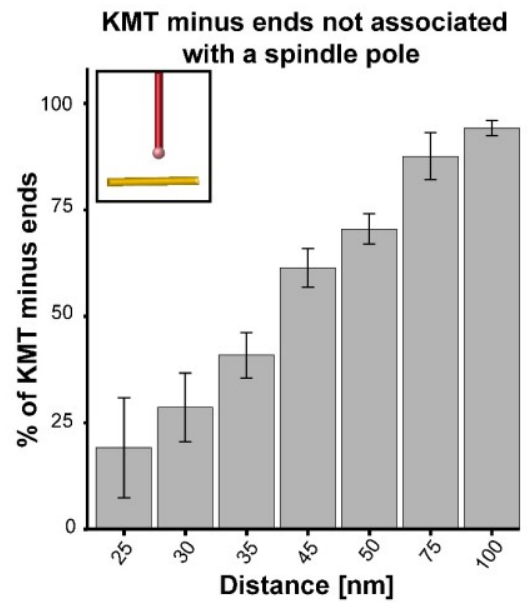

F

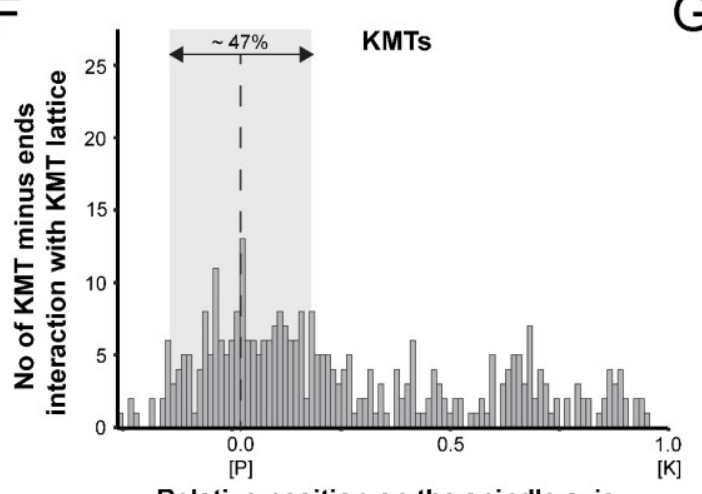

Relative position on the spindle axis

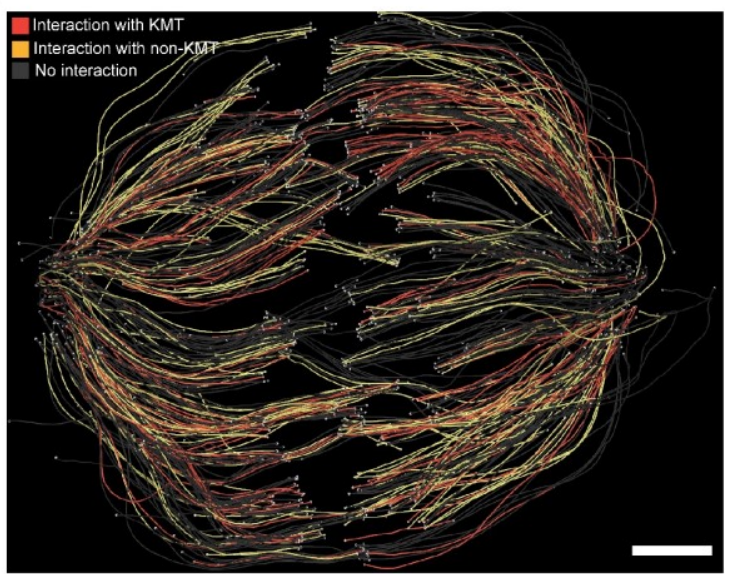

$\mathrm{E}$

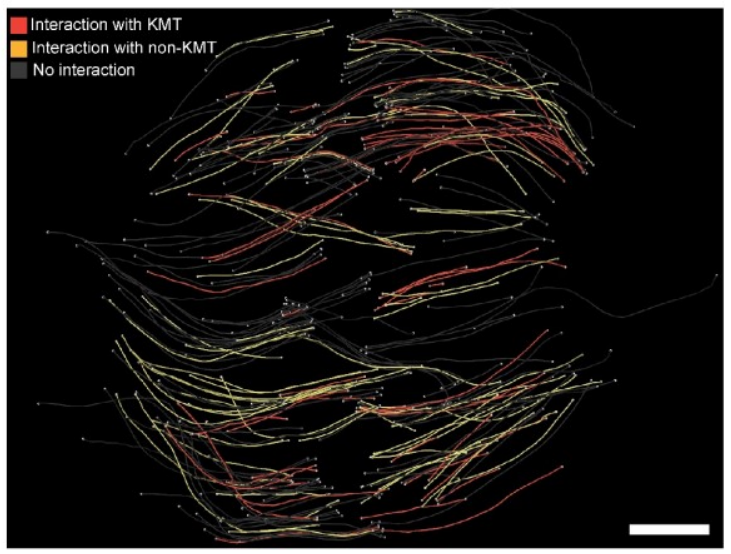

G

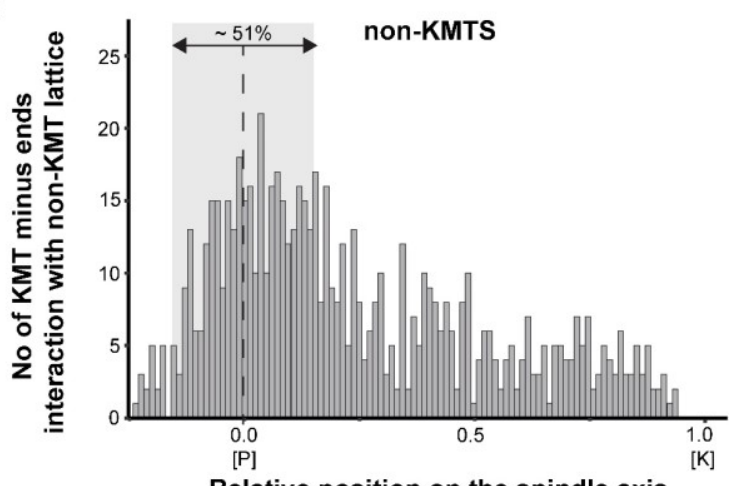




\section{Figure 7. Association of KMT minus ends with the MT network}

1003 (A) Schematic illustration of the association of KMT minus ends with MT lattices showing a 1004 KMT (red line) with its minus end (red sphere) located close to the lattice of a non-KMT (yellow 1005 line). The distance from a KMT minus end to the center of a non-KMT lattice is estimated. (B) 1006 Bar plot showing the percentage of all KMT minus ends associated with a MT lattice depending 1007 on defined distances of association (25-100 nm). The standard deviations are given. (C) A 1008 three-dimensional model showing the interaction pattern of all KMT minus ends associated 1009 with MTs based on an interaction distance of $35 \mathrm{~nm}$ (data obtained from spindle \#1). KMTs 1010 with their minus end associated with other KMTs are labeled in red, KMTs with an association 1011 to non-KMTs are marked in yellow. KMTs without any association to other MTs are shown in 1012 grey. (D) Bar plot showing the percentage of KMT minus ends not associated with the 1013 centrosome interaction area. The association with MT lattices is shown in relation to the 1014 chosen distance of association. (E) Three-dimensional model of KMTs with their minus ends 1015 not attached to the centrosome and associated with MT lattices. Colors are shown as 1016 displayed in C. (F) Graph showing the number of KMT minus ends interacting with KMT 1017 lattices within a distance of $35 \mathrm{~nm}$. The number of KMT minus ends is plotted against the 1018 relative position on the spindle axis ( $\mathrm{P}$, pole; $\mathrm{K}$, kinetochore). (G) Bar plot showing the number 1019 of KMT minus ends interacting with non-KMT lattices within a distance of $35 \mathrm{~nm}$. The graph 1020 gives the relative position of the non-KMT minus ends plotted against the relative position on 1021 the pole axis. Data from the three reconstructed spindles are shown. Scale bars, $1 \mu \mathrm{m}$ 
A

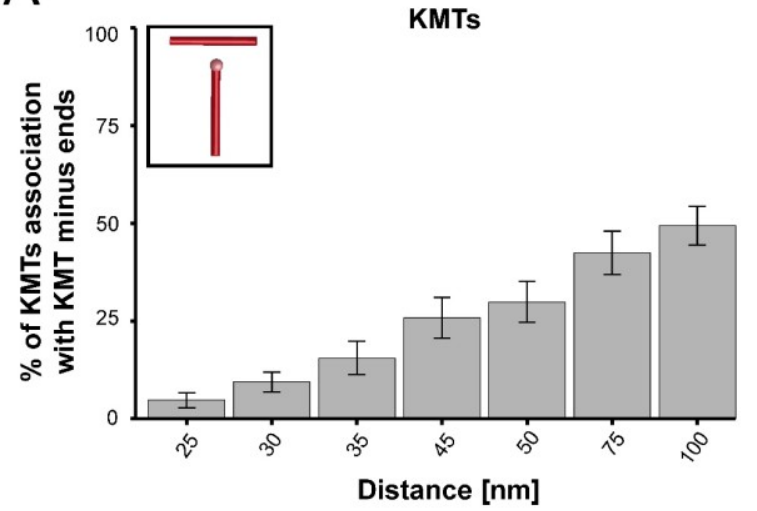

B

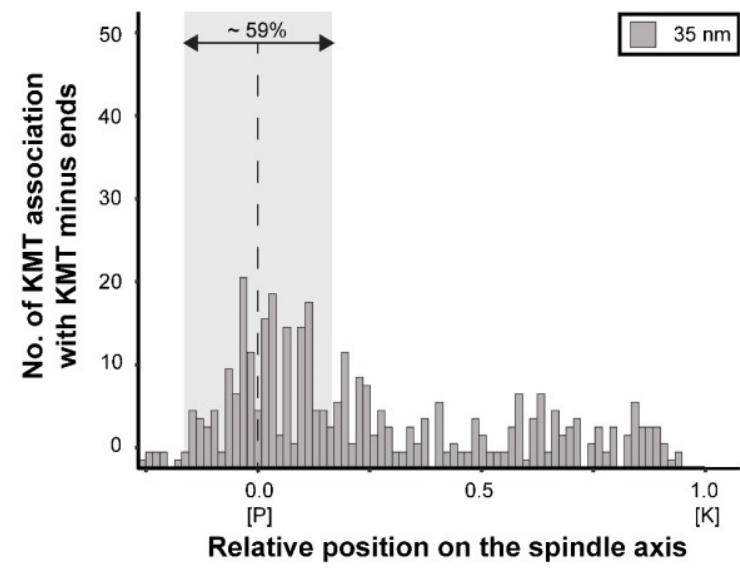

C

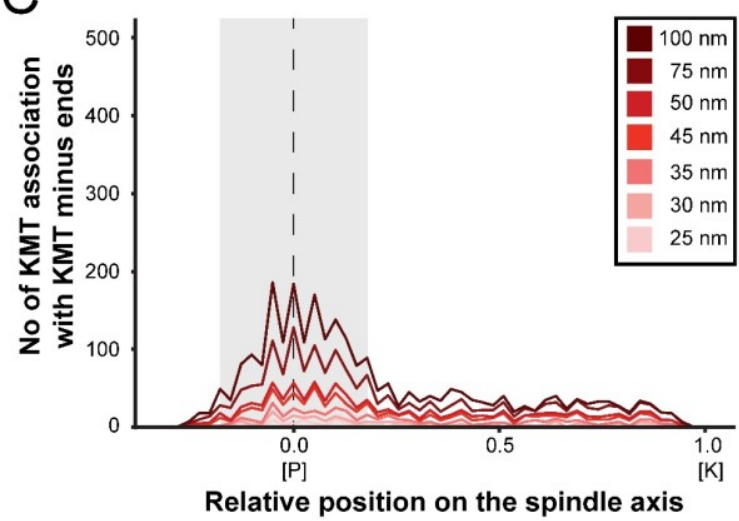

D

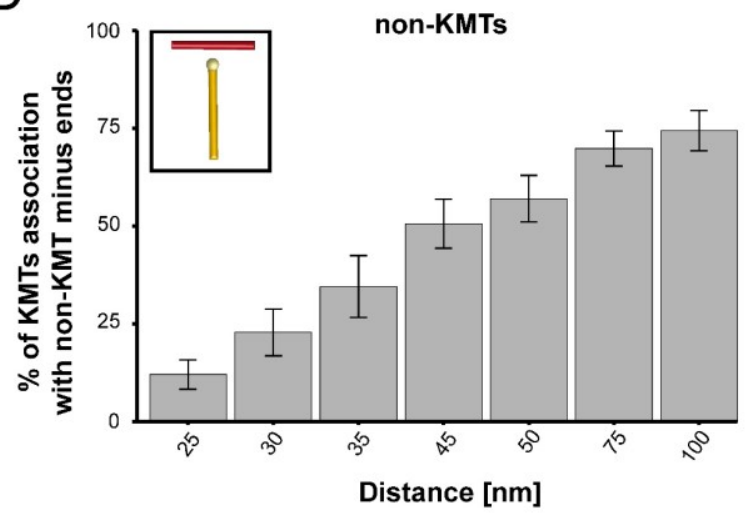

$E$

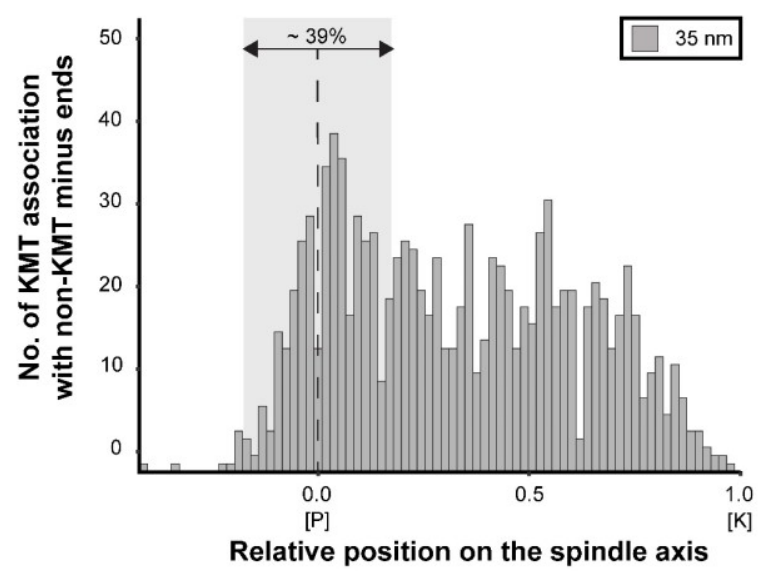

$\mathrm{F}$

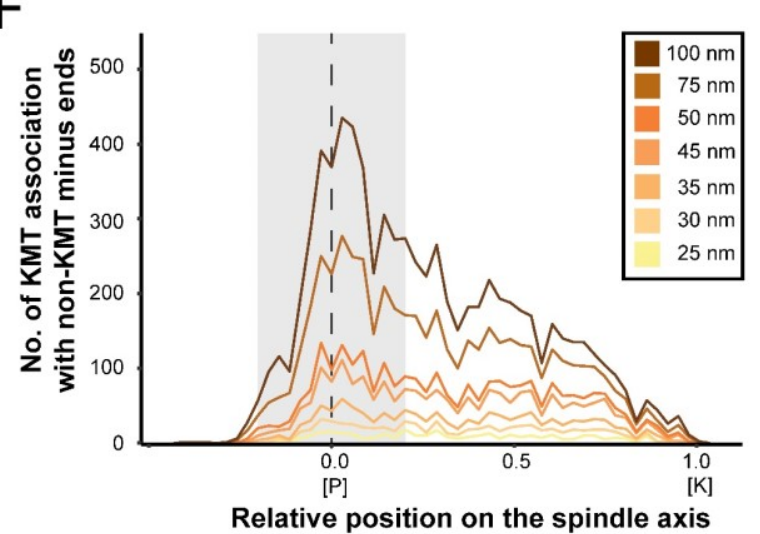

Figure 7-figure supplement 1. Association of KMT lattices with MT minus ends

(A) Bar plot showing the percentage of KMT lattices associated with other KMT minus ends depending on defined interaction distances. The standard deviations are indicated. (B) Graph showing the number of KMT lattices associated with other KMT minus ends plotted along with the relative position on the spindle axis ( $\mathrm{P}$, pole; $\mathrm{K}$, kinetochore). The distance of MT interaction is $35 \mathrm{~nm}$. The percentage of KMT association at the MT-centrosome interaction 
1030 area is indicated. (C) Plot showing the number of KMT lattices associated with KMT minus 1031 ends as shown in B. The plot shows the number of associations for distances of $25,30,35$, $103245,50,75$ and $100 \mathrm{~nm}$. (D) Bar plot showing the percentage of KMT lattices associated with 1033 non-KMT minus ends. The distance of MT interaction is $35 \mathrm{~nm}$. (E) Line plot showing the 1034 number of KMT lattices associated with other non-KMT minus ends plotted against the relative 1035 position on the spindle axis. The percentage of KMT association at the MT-centrosome 1036 interaction area is indicated. All graphs show mean values from the three reconstructed 1037 spindles. (F) Plot showing the number of non-KMT lattices associated with KMT minus ends 1038 as shown in $\mathrm{E}$. The plot shows the number of associations for distances of $25,30,35,45,50$, 103975 and $100 \mathrm{~nm}$. 


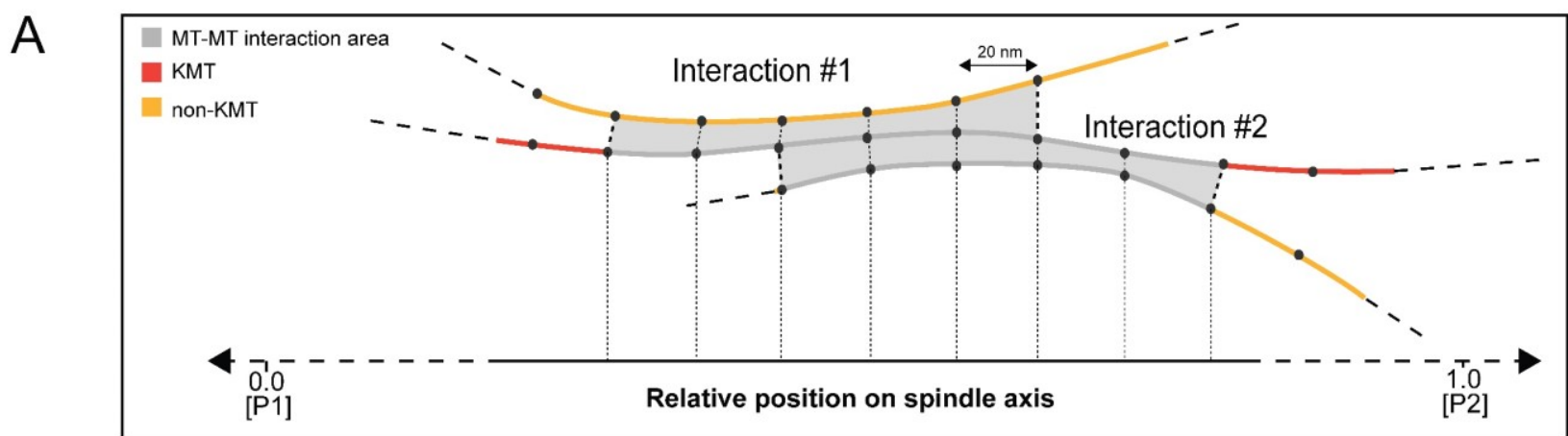

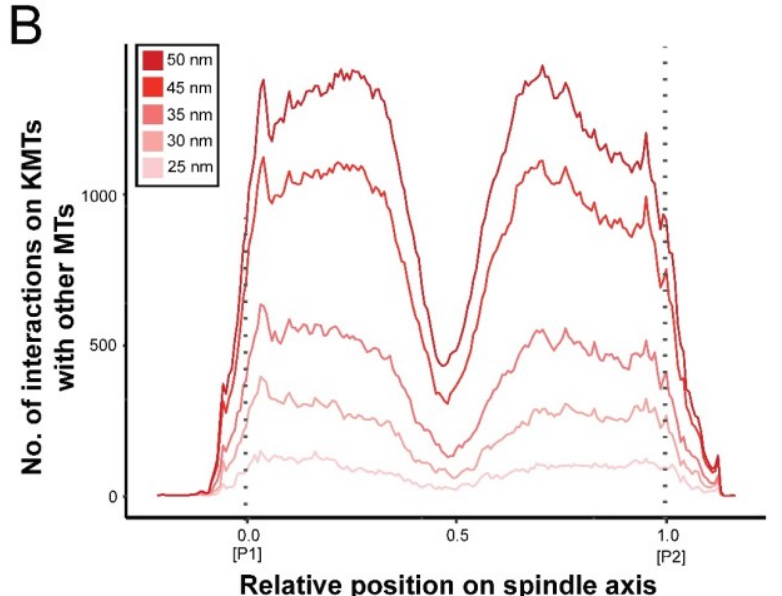

C

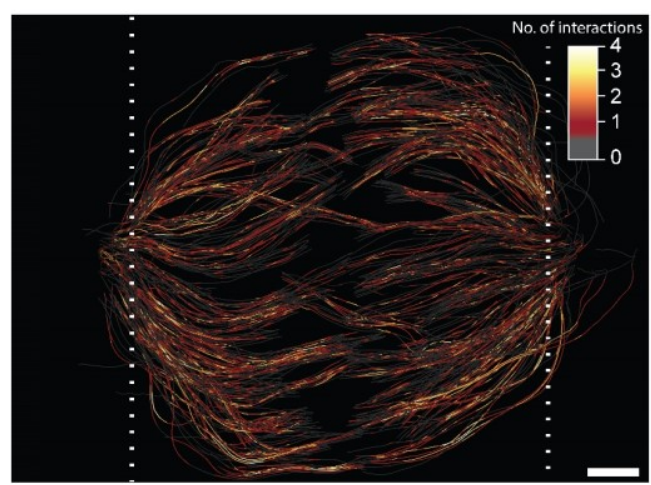

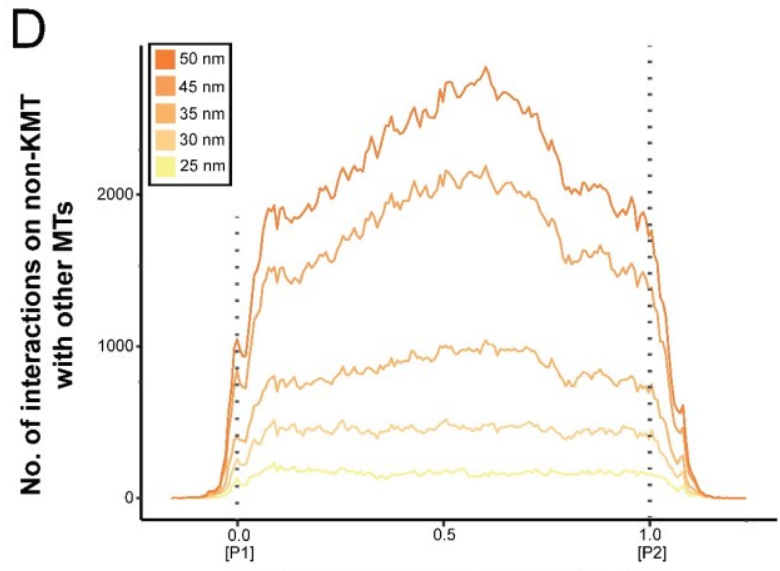

E

\section{Figure 8. Positions of MT-MT associations}

1042 (A) Schematic illustration of MT-MT associations. The interactions are given in intervals of 20 $1043 \mathrm{~nm}$. This allows mapping of the number of interactions on the spindle pole axis. KMTs are illustrated in red, non-KMTs in yellow, areas of MT-MT association in grey. (B) Plot showing number of KMTs associated with other MTs plotted against the relative position of the association on a spindle axis. (C) 3D model of all KMTs showing regions of MT-MT association within a distance of $35 \mathrm{~nm}$ or closer. Each KMT segment is color-coded according to the number of associations. (D) The number of non-KMTs associated with neighboring MTs plotted against the relative position of the association on a spindle axis. Plots are shown for 
bioRxiv preprint doi: https://doi.org/10.1101/2021.11.13.468347; this version posted November 13, 2021. The copyright holder for this preprint (which was not certified by peer review) is the author/funder. All rights reserved. No reuse allowed without permission.

1050 five different thresholds of MT-MT association distances. (E) 3D model of all non-KMTs 1051 showing regions of MT-MT association within a distance of $35 \mathrm{~nm}$ or closer. Scale bars, $1 \mu \mathrm{m}$. 
A

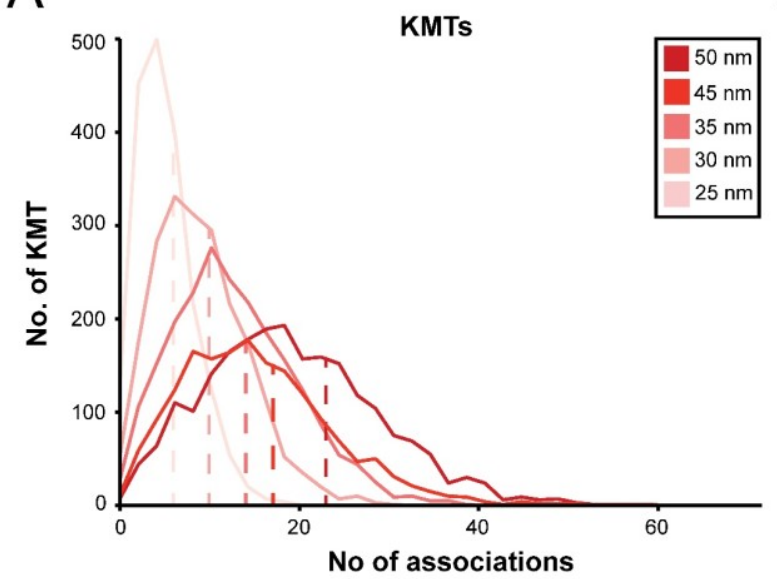

C

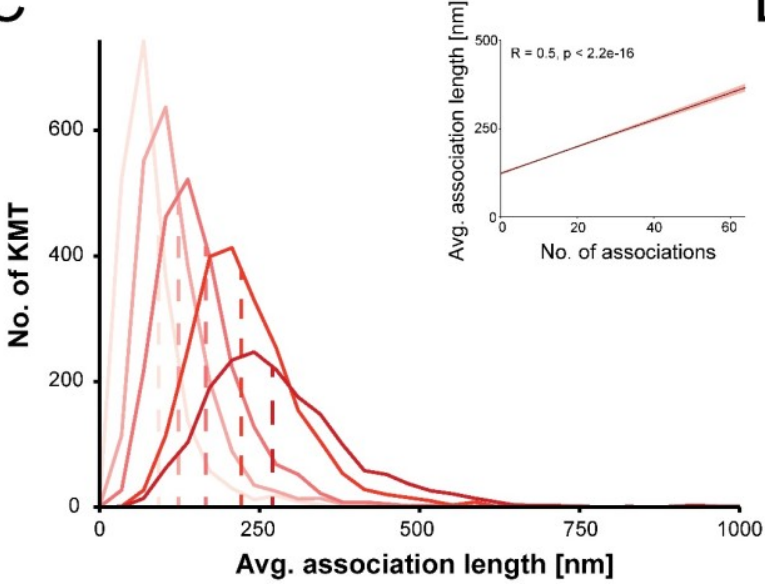

B

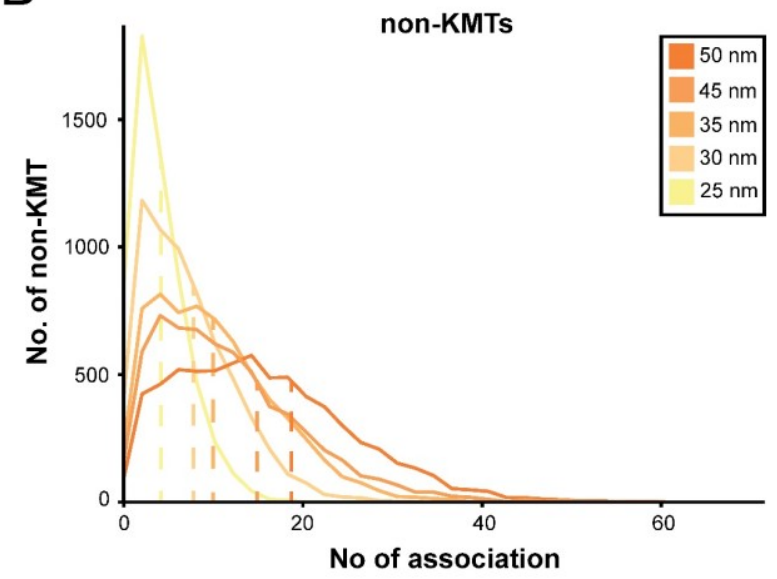

$\mathrm{D}$

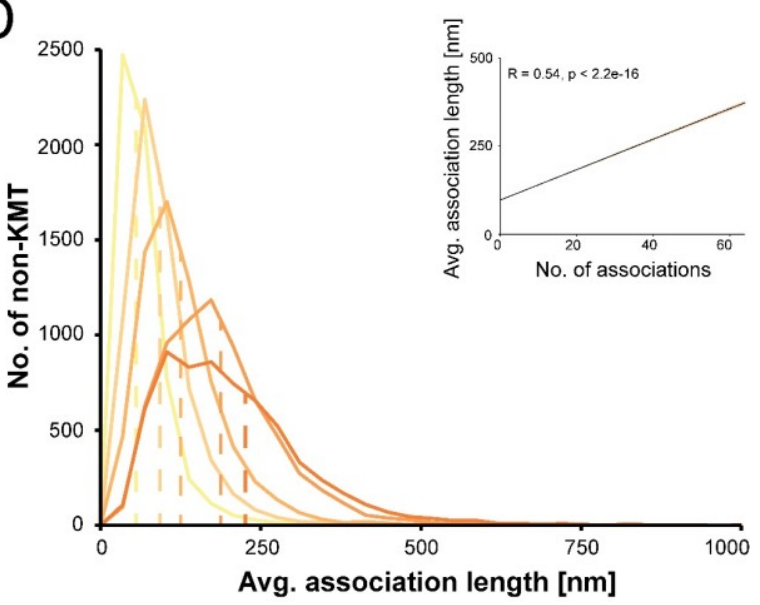

1053 Figure 8-figure supplement 1. Association of KMT lattices with MT minus ends

1055 (A) Number of KMTs plotted against the number of associations with other MTs in the spindle per individual KMT. Plots are shown for five different distances between MT-MTs. (B) Number of KMTs plotted against the average length of MT-MT association per individual KMT. (C) The number of non-KMTs plotted against the number of associations. Plots are shown for five different thresholds of MT-MT association distances. (D) The number of non-KMTs plotted against the average length of MT-MT association per individual non-KMT. A correlation plot is also shown for the number of MT associations and length for KMTs and non-KMTs. 

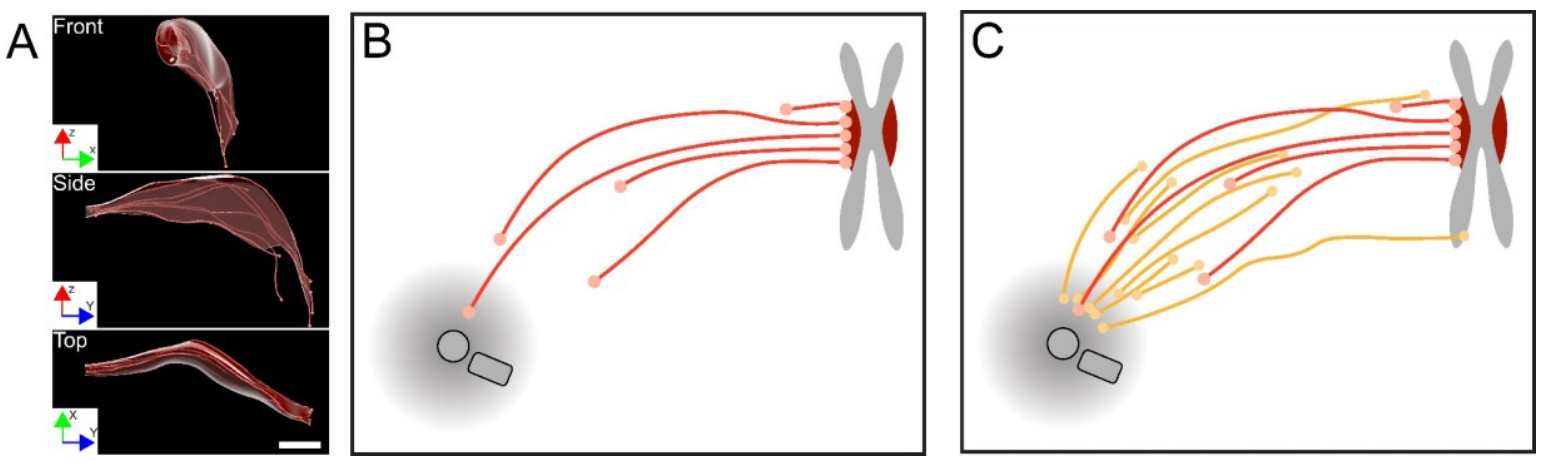

Figure 9. Model of a k-fiber showing a semi-direct connection between a

\section{kinetochore and spindle poles}

1065 (A) Three-dimensional model of a selected k-fiber with an overlay k-fiber area drawn around KMTs using the alpha shape method. The same k-fiber is shown from three different perspectives (front, side and top view).

(B) Schematic model of a semi-direct connection between a kinetochore (chromosome in grey with paired kinetochores in dark red) and a spindle pole (with centered centrioles) as established for a single k-fiber. KMTs are shown in red. MT ends are indicated as spheres. (C) Schematic model of the k-fiber interaction with a non-KMTs network. KMTs are shown in red, non-KMTS in yellow, chromosomes with paired kinetochores in dark red and a spindle pole with centrioles in grey. MTs ends are indicated as spheres. Scale bar, $1 \mu \mathrm{m}$. 
1074 Movie Supplements

1075 Figure 2-movie supplement 1. Generation of a 3D model from joined serial electron tomograms displaying spindle \#1

1077 This video shows a series of stitched 3D reconstructions of spindle \#1 and the corresponding 1078 3D model. The stacking of serial tomograms to increase the tomographic volume is illustrated. 1079 The MTs are shown in white lines, chromosomes are illustrated in blue. This movie 1080 corresponds to Figure 2C.

1081 Figure 2-movie supplement 2. Generation of a 3D model from joined serial 1082 electron tomograms displaying spindle \#2

This video shows a series of stitched 3D reconstructions of spindle \#2 and the corresponding 3D model. The stacking of serial tomograms to increase the tomographic volume is illustrated. The MTs are shown in white lines, chromosomes are illustrated in blue. This movie corresponds to Figure 2D.

Figure 2-movie supplement 3. Generation of a 3D model from joined serial electron tomograms displaying spindle \#3

1089 This video shows a series of stitched 3D reconstructions of spindle \#3 and the corresponding 3D model. The stacking of serial tomograms to increase the tomographic volume is illustrated. The MTs are shown in white lines, chromosomes are illustrated in blue. This movie corresponds to Figure 2E.

Figure 2-movie supplement 4. Movie showing an overview of KMTs in

\section{spindle \#1}

This video shows a $3 \mathrm{D}$ model of the reconstructed metaphase spindle \#1. Non-KMTs are shown in yellow, the KMTs are shown in red, chromosomes are illustrated in blue and centrioles in grey. This movie corresponds to Figure 2F. 
1100 This video shows a 3D model of the reconstructed metaphase spindle \#1. Non-KMTs are shown in yellow, the KMTs are shown in red, chromosomes are illustrated in blue and centrioles in grey. This movie corresponds to Figure 2G.

Figure 2-movie supplement 6. Movie showing an overview of KMTs in spindle \#3

1105

This video shows a 3D model of the reconstructed metaphase spindle \#1. Non-KMTs are shown in yellow, the KMTs are shown in red, chromosomes are illustrated in blue and centrioles in grey. This movie corresponds to Figure $2 \mathrm{H}$.

1108 Figure 3-movie supplement 1. Movie of the 3D reconstruction of a single 1109 k-fiber

1110 This video shows a 3D model of the selected sister k-fibers of spindle \#1. The KMTs are shown 1111 in red, the KMT ends by white spheres, the centrioles in grey. This movie corresponds to 1112 Figure $3 \mathrm{~A}(\# \mathrm{I})$.

1113 Figure 3-movie supplement 2. Movie of the 3D reconstruction of a single 1114 k-fiber

1115 This video shows a 3D model of the selected sister k-fibers of spindle \#1. The KMTs are shown 1116 in red, the KMT ends by white spheres, the centrioles in grey. This movie corresponds to 1117 Figure $3 \mathrm{~A}(\# \mathrm{II})$.

1118 Figure 3-movie supplement 3. Movie of the 3D reconstruction of a single 1119 k-fiber

1120 This video shows a 3D model of the selected sister k-fibers of spindle \#1. The KMTs are shown 1121 in red, the KMT ends by white spheres, the centrioles in grey. This movie corresponds to 1122 Figure 3A (\# III). 
This video shows a 3D model of the selected sister k-fibers of spindle \#1. The KMTs are shown in red, the KMT ends by white spheres, the centrioles in grey. This movie corresponds to Figure 3A (\# IV).

Figure 3-movie supplement 5. Movie of the 3D reconstruction of a single k-fiber

This video shows a 3D model of the selected sister $k$-fibers of spindle \#1. The KMTs are shown in red, the KMT ends by white spheres, the centrioles in grey. This movie corresponds to Figure $3 \mathrm{~A}(\# \mathrm{~V})$.

Figure 3-movie supplement 6. Movie of the 3D reconstruction of a single k-fiber

This video shows a 3D model of the selected sister k-fibers of spindle \#1. The KMTs are shown in red, the KMT ends by white spheres, the centrioles in grey. This movie corresponds to Figure 3A (\# VI).

Figure 5-movie supplement 1 . Movie showing the analysis of $k$-fiber tortuosity in spindle \#1

1140 This video shows a 3D model of all KMTs with indicated global tortuosity of spindle \#1. KMTs are shown in color coding as indicated in Figure 5C. KMT ends are shown as white spheres, centrioles in grey. This movie corresponds to Figure 5C.

1143 Figure 5-movie supplement 2. Movie showing the analysis of k-fiber tortuosity in spindle \#2

This video shows a 3D model of all KMTs with indicated global tortuosity of spindle \#1. KMTs are shown in color coding as indicated in Figure 5C. KMT ends are shown as white spheres, centrioles in grey. This movie corresponds to Figure 5C (example of spindle \#2 shown as a movie only). 
1149 Figure 5-movie supplement 3 . Movie showing the analysis of k-fiber 1150 tortuosity in spindle \#3

1151 This video shows a 3D model of all KMTs with indicated global tortuosity of spindle \#1. KMTs 1152 are shown in color coding as indicated in Figure 5C. KMT ends are shown as white spheres, 1153 centrioles in grey. This movie corresponds to Figure 5C (example of spindle \#3 shown as a 1154 movie only).

1155 Figure 7-movie supplement 1. Movie showing associations of KMT minus 1156 ends with MT lattices in spindle \#1

1157 This video shows a 3D model of all KMTs from spindle \#1. KMT minus ends interacting with 1158 other MTs with association distances ranging from 25 to $100 \mathrm{~nm}$ are shown. KMT minus ends 1159 interacting with either KMT or non-KMT lattices are indicated in red or yellow, respectively. 1160 KMTs without any interactions are shown in grey. This movie corresponds to Figure 7C.

1161 Figure 8-movie supplement 1. Movie showing associations of KMT lattices with neighbor MTs

1163 This video shows a 3D model of all KMTs in spindle \#1. KMT lattices interacting with other MT 1164 lattices within MT distances ranging from 25 to $50 \mathrm{~nm}$ are shown. KMTs are shown as grey 1165 lines with color-coded regions showing the number of MT-MT associations. This movie 1166 corresponds to Figure 8C.

1167 Figure 8-movie supplement 2. Movie showing associations of non-KMTs 1168 with neighbor MTs

1169 This video shows a 3D model of all non-KMTs showing regions of MT-MT associations in spindle \#1 over increasing MT distances ranging from 25 to $50 \mathrm{~nm}$. Non-KMTs are shown as grey lines with color-coded regions showing the number of MT-MT associations. This movie corresponds to Figure 8E. 


\section{Tables}

1174 Table 1. Characterization of the 3D-reconstructed metaphase spindles in 1175 HeLa cells

\begin{tabular}{|c|c|c|c|c|c|c|c|}
\hline Data set & $\begin{array}{c}\text { Spindle pole } \\
\text { distance } \\
{[\mu \mathrm{m}]}\end{array}$ & $\begin{array}{c}\text { Inter- } \\
\text { kinetochore } \\
\text { distance }[\mu \mathrm{m}]\end{array}$ & $\begin{array}{l}\text { Total } \\
\text { no. of } \\
\text { MTs }\end{array}$ & $\begin{array}{c}\text { No. of } \\
\text { kinetochores }\end{array}$ & $\begin{array}{l}\text { No. of } \\
\text { KMTs }\end{array}$ & $\begin{array}{l}\text { No. of } \\
\text { non- } \\
\text { KMTs }\end{array}$ & $\begin{array}{c}\text { No. of } \\
\text { k-fibers }\end{array}$ \\
\hline $\begin{array}{l}\text { Spindle } \\
\# 1\end{array}$ & 7.16 & $\begin{array}{c}1.07 \pm 0.20 \\
(n=43)\end{array}$ & 4884 & 92 & $\begin{array}{c}797 \\
(16.3 \%)\end{array}$ & $\begin{array}{c}4087 \\
(83.7 \%)\end{array}$ & 92 \\
\hline $\begin{array}{l}\text { Spindle } \\
\text { \#2 }\end{array}$ & 10.39 & $\begin{array}{c}1.21 \pm 0.21 \\
(n=50)\end{array}$ & 8047 & 110 & $\begin{array}{c}1102 \\
(13.7 \%)\end{array}$ & $\begin{array}{c}6945 \\
(86.3 \%)\end{array}$ & 110 \\
\hline $\begin{array}{c}\text { Spindle } \\
\text { \#3 }\end{array}$ & 9.48 & $\begin{array}{c}1.02 \pm 0.29 \\
(n=40)\end{array}$ & 5904 & 90 & $\begin{array}{c}680 \\
(11.5 \%)\end{array}$ & $\begin{array}{c}5224 \\
(88.5 \%)\end{array}$ & 90 \\
\hline
\end{tabular}


1177 Table 2. Quantitative analysis of KMTs and non-KMTs

\begin{tabular}{|c|c|c|c|c|c|c|c|}
\hline Analysis set & $\begin{array}{l}\text { Length } \\
\text { of } \\
\text { KMTs } \\
{[\mu \mathrm{m}]}\end{array}$ & $\begin{array}{c}\text { Length } \\
\text { of non- } \\
\text { KMTs } \\
{[\mu \mathrm{m}]}\end{array}$ & $\begin{array}{l}\text { No. of KMTs } \\
\text { per } \\
\text { kinetochore }\end{array}$ & $\begin{array}{l}\text { No. of KMTs } \\
\text { at } \\
\text { centrosome } \\
\text { interaction } \\
\text { area }\end{array}$ & $\begin{array}{c}\text { Mean KMT } \\
\text { minus end } \\
\text { distance } \\
\text { to poles } \\
{[\mu \mathrm{m}]}\end{array}$ & $\begin{array}{c}\text { No. of KMTs } \\
\text { associated } \\
\text { with poles } \\
\text { [\%] }\end{array}$ & $\begin{array}{l}\text { No. of non- } \\
\text { KMTs } \\
\text { associated } \\
\text { with poles } \\
\text { [\%] }\end{array}$ \\
\hline $\begin{array}{c}\text { Spindle } \\
\# 1\end{array}$ & $\begin{array}{c}3.23 \\
( \pm 1.49)\end{array}$ & $\begin{array}{c}2.03 \\
( \pm 1.6)\end{array}$ & $\begin{array}{c}8.04 \\
( \pm 1.86)\end{array}$ & $\begin{array}{c}4.1 \\
( \pm 1.8)\end{array}$ & 1.16 & 62.2 & 44.5 \\
\hline $\begin{array}{c}\text { Spindle } \\
\# 2\end{array}$ & $\begin{array}{c}3.69 \\
( \pm 1.87)\end{array}$ & $\begin{array}{c}1.85 \\
( \pm 1.55)\end{array}$ & $\begin{array}{c}9.75 \\
( \pm 2.18)\end{array}$ & $\begin{array}{c}2.4 \\
( \pm 2.0)\end{array}$ & 2.47 & 53.6 & 28.8 \\
\hline $\begin{array}{c}\text { Spindle } \\
\# 3\end{array}$ & $\begin{array}{c}4.03 \\
( \pm 1.79)\end{array}$ & $\begin{array}{c}1.91 \\
( \pm 1.80)\end{array}$ & $\begin{array}{c}7.49 \\
( \pm 1.91)\end{array}$ & $\begin{array}{c}3.4 \\
( \pm 1.8)\end{array}$ & 1.35 & 62.0 & 42.3 \\
\hline
\end{tabular}


1179 Table 3. Quantitative analysis of the k-fiber organization

\begin{tabular}{|c|c|c|c|c|c|c|}
\hline $\begin{array}{l}\text { Data set } \\
\text { Analysis }\end{array}$ & $\begin{array}{c}\text { KMT density at } \\
\text { the kinetochore } \\
{\left[\mathrm{KMT}^{\mathrm{K}} / \mathrm{mm}^{2}\right]}\end{array}$ & $\begin{array}{c}\text { Neighborhood } \\
\text { KMT-KMT distance } \\
\text { at the kinetochore } \\
{[\mathrm{nm}]}\end{array}$ & $\begin{array}{l}\text { Tortuosity } \\
\text { of KMTs }\end{array}$ & $\begin{array}{c}\text { Percentage } \\
\text { of curved } \\
\text { KMTs [\%] }\end{array}$ & $\begin{array}{c}\text { Area of k- } \\
\text { fibers } \\
{\left[\mu \mathrm{m}^{2}\right]}\end{array}$ & $\begin{array}{c}\text { Density of } \\
\text { k-fibers } \\
\text { [\%] }\end{array}$ \\
\hline $\begin{array}{c}\text { Spindle } \\
\# 1\end{array}$ & $\begin{array}{c}0.07 \\
( \pm 0.05)\end{array}$ & $\begin{array}{c}61 \\
( \pm 11)\end{array}$ & $\begin{array}{c}1.09 \\
( \pm 0.10)\end{array}$ & 36.1 & $\begin{array}{c}0.063 \\
( \pm 0.085)\end{array}$ & $\begin{array}{c}34 \\
( \pm 27)\end{array}$ \\
\hline $\begin{array}{c}\text { Spindle } \\
\# 2\end{array}$ & $\begin{array}{c}0.09 \\
( \pm 0.06)\end{array}$ & $\begin{array}{c}65 \\
( \pm 12)\end{array}$ & $\begin{array}{c}1.06 \\
( \pm 0.06)\end{array}$ & 21.4 & $\begin{array}{c}0.068 \\
( \pm 0.092)\end{array}$ & $\begin{array}{c}70 \\
( \pm 25)\end{array}$ \\
\hline $\begin{array}{c}\text { Spindle } \\
\# 3\end{array}$ & $\begin{array}{c}0.06 \\
( \pm 0.04)\end{array}$ & $\begin{array}{c}66 \\
( \pm 12)\end{array}$ & $\begin{array}{c}1.11 \\
( \pm 0.11)\end{array}$ & 39.5 & $\begin{array}{c}0.080 \\
( \pm 0.150)\end{array}$ & $\begin{array}{c}59 \\
( \pm 39)\end{array}$ \\
\hline
\end{tabular}


1181 Table 4. Analysis of the potential association of KMT minus ends with 1182 other neighboring KMT lattices

\begin{tabular}{|c|c|c|c|c|c|c|c|c|}
\hline \multirow{2}{*}{$\begin{array}{c}\text { Data } \\
\text { set }\end{array}$} & \multirow{2}{*}{ Analysis } & \multicolumn{7}{|c|}{ Association distance $[\mathrm{nm}]$} \\
\hline & & 25 & 30 & 35 & 45 & 50 & 75 & 100 \\
\hline \multirow{2}{*}{$\begin{array}{l}\text { Spindle } \\
\# 1\end{array}$} & No. of KMTs & 50 & 86 & 141 & 229 & 263 & 317 & 333 \\
\hline & $\begin{array}{l}\text { Percentage of } \\
\text { KMTs [\%] }\end{array}$ & 6.7 & 11.5 & 18.9 & 30.6 & 35.2 & 42.4 & 44.5 \\
\hline \multirow{2}{*}{$\begin{array}{c}\text { Spindle } \\
\# 2\end{array}$} & No. of KMTs & 32 & 67 & 108 & 181 & 213 & 290 & 299 \\
\hline & $\begin{array}{l}\text { Percentage of } \\
\text { KMTs [\%] }\end{array}$ & 3.0 & 6.3 & 10.1 & 16.9 & 19.9 & 27.1 & 27.9 \\
\hline \multirow{2}{*}{$\begin{array}{c}\text { Spindle } \\
\# 3\end{array}$} & No. of KMTs & 24 & 54 & 87 & 145 & 166 & 201 & 218 \\
\hline & $\begin{array}{l}\text { Percentage of } \\
\text { KMTs [\%] }\end{array}$ & 3.6 & 8.0 & 13.0 & 21.5 & 24.6 & 29.8 & 32.3 \\
\hline
\end{tabular}


1184 Table 5. Analysis of the potential association of KMT minus ends with

1185 other neighboring non-KMT lattices

1186

\begin{tabular}{|c|c|c|c|c|c|c|c|c|}
\hline \multirow{2}{*}{$\begin{array}{c}\text { Data } \\
\text { set }\end{array}$} & \multirow{2}{*}{ Analysis } & \multicolumn{7}{|c|}{ Association distance $[\mathrm{nm}]$} \\
\hline & & 25 & 30 & 35 & 45 & 50 & 75 & 100 \\
\hline \multirow{2}{*}{$\begin{array}{c}\text { Spindle } \\
\# 1\end{array}$} & $\begin{array}{l}\text { No. of } \\
\text { KMTs }\end{array}$ & 63 & 110 & 172 & 244 & 278 & 362 & 386 \\
\hline & $\begin{array}{c}\text { Percentage } \\
\text { KMT [\%] }\end{array}$ & 8.4 & 14.7 & 23.0 & 32.6 & 37.2 & 48.4 & 51.6 \\
\hline \multirow{2}{*}{$\begin{array}{c}\text { Spindle } \\
\# 2\end{array}$} & $\begin{array}{l}\text { No. of } \\
\text { KMTs }\end{array}$ & 272 & 343 & 401 & 533 & 578 & 703 & 739 \\
\hline & $\begin{array}{c}\text { Percentage } \\
\text { KMT [\%] }\end{array}$ & 25.5 & 32.0 & 37.4 & 49.7 & 53.9 & 65.6 & 68.9 \\
\hline \multirow{2}{*}{$\begin{array}{c}\text { Spindle } \\
\# 3\end{array}$} & $\begin{array}{l}\text { No. of } \\
\text { KMTs }\end{array}$ & 55 & 100 & 148 & 244 & 280 & 384 & 427 \\
\hline & $\begin{array}{c}\text { Percentage } \\
\text { KMT [\%] }\end{array}$ & 8.2 & 14.8 & 22.0 & 36.2 & 41.5 & 57.0 & 63.4 \\
\hline
\end{tabular}


1188 Table 6. Analysis of the potential association of KMT lattices with other

1189 neighboring KMT minus ends

1190

\begin{tabular}{|c|c|c|c|c|c|c|c|}
\hline \multirow{2}{*}{ Data set } & \multicolumn{7}{|c|}{ Number of associations according to the given association distances [nm] } \\
\hline & 25 & 30 & 35 & 45 & 50 & 75 & 100 \\
\hline Spindle \#1 & 54 & 102 & 184 & 342 & 412 & 733 & 1073 \\
\hline Spindle \#2 & 41 & 88 & 146 & 298 & 379 & 703 & 946 \\
\hline Spindle \#3 & 24 & 60 & 106 & 197 & 241 & 409 & 619 \\
\hline
\end{tabular}

1191 
1192 Table 7. Analysis of the potential association of KMT lattices with other

1193 neighboring non-KMT minus ends

1194

\begin{tabular}{|l|c|c|c|c|c|c|c|}
\hline \multirow{2}{*}{ Data set } & \multicolumn{7}{|c|}{ Number of associations according to the given MT-MT distances [nm] } \\
\cline { 2 - 8 } & $\mathbf{2 5}$ & $\mathbf{3 0}$ & $\mathbf{3 5}$ & $\mathbf{4 5}$ & $\mathbf{5 0}$ & $\mathbf{7 5}$ & $\mathbf{1 0 0}$ \\
\hline & & & & & & & \\
\hline & 141 & 265 & 438 & 771 & 932 & 1733 & 2530 \\
\hline Spindle \#1 & 96 & 194 & 357 & 736 & 956 & 1961 & 2852 \\
\hline Spindle \#3 \#2 & 88 & 213 & 352 & 636 & 798 & 1470 & 2205 \\
\hline
\end{tabular}


Table 8. The average number of associations with MT lattices

1197

\begin{tabular}{|c|c|c|c|c|c|c|}
\hline \multirow{2}{*}{ Data set } & \multirow{2}{*}{ MT type } & \multicolumn{5}{|c|}{ Number of associations according to the given MT-MT distances [nm] } \\
\hline & & 25 & 30 & 35 & 45 & 50 \\
\hline \multirow{2}{*}{$\begin{array}{c}\text { Spindle } \\
\# 1\end{array}$} & KMTs & $\begin{array}{c}3.4 \\
( \pm 2.0)\end{array}$ & $\begin{array}{c}5.7 \\
( \pm 3.1)\end{array}$ & $\begin{array}{c}7.7 \\
( \pm 4.1)\end{array}$ & $\begin{array}{c}10.9 \\
( \pm 5.5)\end{array}$ & $\begin{array}{c}12.5 \\
( \pm 6.3)\end{array}$ \\
\hline & non-KMTs & $\begin{array}{c}2.9 \\
( \pm 2.1)\end{array}$ & $\begin{array}{c}4.4 \\
( \pm 3.4)\end{array}$ & $\begin{array}{c}5.8 \\
( \pm 4.5)\end{array}$ & $\begin{array}{c}8.1 \\
( \pm 6.2)\end{array}$ & $\begin{array}{c}9.2 \\
( \pm 7.0)\end{array}$ \\
\hline \multirow{2}{*}{$\begin{array}{c}\text { Spindle } \\
\# 2\end{array}$} & KMTs & $\begin{array}{c}2.8 \\
( \pm 1.8)\end{array}$ & $\begin{array}{c}4.1 \\
( \pm 2.7)\end{array}$ & $\begin{array}{c}5.6 \\
( \pm 3.5)\end{array}$ & $\begin{array}{c}8.8 \\
( \pm 5.2)\end{array}$ & $\begin{array}{c}10.3 \\
( \pm 6.0)\end{array}$ \\
\hline & non-KMTs & $\begin{array}{c}2.0 \\
( \pm 1.3)\end{array}$ & $\begin{array}{c}2.7 \\
( \pm 1.9)\end{array}$ & $\begin{array}{c}3.6 \\
( \pm 2.7)\end{array}$ & $\begin{array}{c}5.4 \\
( \pm 4.1)\end{array}$ & $\begin{array}{c}6.3 \\
( \pm 4.7)\end{array}$ \\
\hline \multirow{2}{*}{$\begin{array}{c}\text { Spindle } \\
\# 3\end{array}$} & KMTs & $\begin{array}{c}3.3 \\
( \pm 2.0)\end{array}$ & $\begin{array}{c}6.0 \\
( \pm 3.3)\end{array}$ & $\begin{array}{c}7.8 \\
( \pm 4.2)\end{array}$ & $\begin{array}{c}11.0 \\
( \pm 5.6)\end{array}$ & $\begin{array}{c}12.6 \\
( \pm 5.3)\end{array}$ \\
\hline & non-KMTs & $\begin{array}{c}2.5 \\
( \pm 1.9)\end{array}$ & $\begin{array}{c}3.8 \\
( \pm 3.2)\end{array}$ & $\begin{array}{c}4.9 \\
( \pm 4.1)\end{array}$ & $\begin{array}{c}6.8 \\
( \pm 5.6)\end{array}$ & $\begin{array}{c}7.7 \\
( \pm 6.3)\end{array}$ \\
\hline \multirow{2}{*}{ Average } & KMTs & $\begin{array}{c}5.4 \\
( \pm 3.1)\end{array}$ & $\begin{array}{c}9.3 \\
( \pm 5.0)\end{array}$ & $\begin{array}{c}14.3 \\
( \pm 6.1)\end{array}$ & $\begin{array}{c}18.8 \\
( \pm 9.8)\end{array}$ & $\begin{array}{c}22.4 \\
( \pm 10.7)\end{array}$ \\
\hline & non-KMTs & $\begin{array}{c}4.4 \\
( \pm 3.0)\end{array}$ & $\begin{array}{c}7.5 \\
( \pm 5.0)\end{array}$ & $\begin{array}{c}10.1 \\
( \pm 6.4)\end{array}$ & $\begin{array}{c}15.7 \\
( \pm 9.5)\end{array}$ & $\begin{array}{c}18.2 \\
( \pm 10.9)\end{array}$ \\
\hline
\end{tabular}


1199 Table 9. The average length of associations with MT lattices

\begin{tabular}{|c|c|c|c|c|c|c|}
\hline \multirow{2}{*}{ Data set } & \multirow{2}{*}{ MT type } & \multicolumn{5}{|c|}{ Length of associations according to the given MT-MT distances [nm] } \\
\hline & & 25 & 30 & 35 & 45 & 50 \\
\hline \multirow{2}{*}{ Spindle \#1 } & KMTs & $\begin{array}{c}103.2 \\
( \pm 89.3)\end{array}$ & $\begin{array}{c}148.5 \\
( \pm 97.3)\end{array}$ & $\begin{array}{c}197.1 \\
( \pm 117.8)\end{array}$ & $\begin{array}{c}265.3 \\
( \pm 118.2)\end{array}$ & $\begin{array}{c}291.3 \\
( \pm 121.3)\end{array}$ \\
\hline & non-KMTs & $\begin{array}{c}65.9 \\
( \pm 46.7)\end{array}$ & $\begin{array}{c}92.4 \\
( \pm 64.2)\end{array}$ & $\begin{array}{c}120.8 \\
( \pm 80.4)\end{array}$ & $\begin{array}{c}169.0 \\
( \pm 105.7)\end{array}$ & $\begin{array}{c}188.0 \\
( \pm 111.71)\end{array}$ \\
\hline \multirow[b]{2}{*}{ Spindle \#2 } & KMTs & $\begin{array}{c}94.2 \\
( \pm 86.8)\end{array}$ & $\begin{array}{c}124.0 \\
( \pm 88.2)\end{array}$ & $\begin{array}{c}164.6 \\
( \pm 104.6)\end{array}$ & $\begin{array}{c}262.5 \\
( \pm 137.1)\end{array}$ & $\begin{array}{c}310.1 \\
( \pm 154.0)\end{array}$ \\
\hline & non-KMTs & $\begin{array}{c}66.6 \\
( \pm 57.9)\end{array}$ & $\begin{array}{c}85.5 \\
( \pm 71.9)\end{array}$ & $\begin{array}{c}107.0 \\
( \pm 85.3)\end{array}$ & $\begin{array}{c}159.7 \\
( \pm 121.4)\end{array}$ & $\begin{array}{c}183.0 \\
( \pm 135.0)\end{array}$ \\
\hline \multirow{2}{*}{ Spindle \#3 } & KMTs & $\begin{array}{c}94.2 \\
( \pm 86.8)\end{array}$ & $\begin{array}{c}124.0 \\
( \pm 88.2)\end{array}$ & $\begin{array}{c}164.6 \\
( \pm 104.6)\end{array}$ & $\begin{array}{c}262.5 \\
( \pm 137.1)\end{array}$ & $\begin{array}{c}310.1 \\
( \pm 154.0)\end{array}$ \\
\hline & non-KMTs & $\begin{array}{c}84.5 \\
( \pm 69.8)\end{array}$ & $\begin{array}{c}137.5 \\
( \pm 101.7)\end{array}$ & $\begin{array}{c}195.1 \\
( \pm 124.0)\end{array}$ & $\begin{array}{c}274.6 \\
( \pm 135.7)\end{array}$ & $\begin{array}{c}299.8 \\
( \pm 137.3)\end{array}$ \\
\hline \multirow{2}{*}{ Average } & KMTs & $\begin{array}{c}83.9 \\
( \pm 56.4)\end{array}$ & $\begin{array}{r}118.5 \\
( \pm 63.8)\end{array}$ & $\begin{array}{c}162.5 \\
( \pm 70.1)\end{array}$ & $\begin{array}{r}234.2 \\
( \pm 97.9)\end{array}$ & $\begin{array}{c}260.8 \\
( \pm 101.7)\end{array}$ \\
\hline & non-KMTs & $\begin{array}{c}67.7 \\
( \pm 44.5)\end{array}$ & $\begin{array}{c}96.1 \\
( \pm 59.2)\end{array}$ & $\begin{array}{c}128.0 \\
( \pm 74.6)\end{array}$ & $\begin{array}{c}186.3 \\
( \pm 93.1)\end{array}$ & $\begin{array}{c}211.1 \\
( \pm 102.7)\end{array}$ \\
\hline
\end{tabular}


Table 10. Tomographic data sets as used throughout this study

\begin{tabular}{|c|c|c|c|c|c|}
\hline $\begin{array}{c}\text { Metadata / } \\
\text { Data set }\end{array}$ & $\begin{array}{l}\text { Original } \\
\text { data set }\end{array}$ & $\begin{array}{c}\text { Montages } \\
(\mathrm{X} / \mathrm{Y})\end{array}$ & $\begin{array}{c}\text { Number of serial } \\
\text { sections [300 } \mathrm{nm} \\
\text { each] }\end{array}$ & $\begin{array}{c}\text { Estimated } \\
\text { tomographic volume } \\
{\left[\mu \mathrm{m}^{3}\right]}\end{array}$ & $\begin{array}{l}\text { Data } \\
\text { size } \\
\text { [Gb] }\end{array}$ \\
\hline Spindle \#1 & T_0475 & $2 \times 3$ & 22 & 598 & 46.5 \\
\hline Spindle \#2 & T_0479 & $2 \times 3$ & 29 & 996 & 77.9 \\
\hline Spindle \#3 & T_0494 & $2 \times 3$ & 35 & 904 & 71.9 \\
\hline
\end{tabular}




\begin{tabular}{|c|c|c|c|c|}
\hline 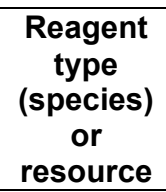 & Designation & Source or reference & Identifiers & $\begin{array}{l}\text { Additional } \\
\text { information }\end{array}$ \\
\hline $\begin{array}{c}\text { Strain, } \\
\text { strain } \\
\text { backgroun } \\
\text { d (HeLa, } \\
\text { Kyoto) }\end{array}$ & Gerlich Lab & IMBA, Vienna, Austria & - & - \\
\hline $\begin{array}{l}\text { Software, } \\
\text { algorithm }\end{array}$ & $\begin{array}{c}\text { SerialEM } \\
\text { Boulder Laboratory for } \\
\text { 3-Dimensional Electron } \\
\text { Microscopy of cells } \\
\text { Colorado }\end{array}$ & $\begin{array}{c}\text { https://bio3d.colorado.edu/ } \\
\text { SerialEM/ }\end{array}$ & - & - \\
\hline $\begin{array}{l}\text { Software, } \\
\text { algorithm }\end{array}$ & $\begin{array}{l}\text { IMOD } \\
\text { Boulder Laboratory for } \\
\text { 3-Dimensional Electron } \\
\text { Microscopy of cells } \\
\text { Colorado }\end{array}$ & $\begin{array}{l}\text { http://bio3d.colorado.edu/ } \\
\text { imod }\end{array}$ & - & - \\
\hline $\begin{array}{l}\text { Software, } \\
\text { algorithm }\end{array}$ & $\begin{array}{c}\text { Amira } \\
\text { Thermo Fisher } \\
\text { Scientific }\end{array}$ & AmiraZibEdition & - & - \\
\hline $\begin{array}{l}\text { Software, } \\
\text { algorithm }\end{array}$ & $\begin{array}{c}\text { ASGA } \\
\text { Robert Kiewisz / } \\
\text { Müller - Reichert Lab } \\
\text { Dresden }\end{array}$ & $\begin{array}{c}\text { https://github.com/RRobert92/ } \\
\text { ASGA }\end{array}$ & - & $\begin{array}{c}\text { https://kiewisz.s } \\
\text { hinyapps.io/AS } \\
\text { GA }\end{array}$ \\
\hline $\begin{array}{l}\text { Software, } \\
\text { algorithm }\end{array}$ & $\begin{array}{l}\text { ASGA - 3D Viewer } \\
\text { Robert Kiewisz / } \\
\text { Müller - Reichert Lab } \\
\text { Dresden }\end{array}$ & $\begin{array}{c}\text { https://github.com/RRobert92/ } \\
\text { ASGA_3DViewer }\end{array}$ & - & $\begin{array}{c}\text { https://cfci.shiny } \\
\text { apps.io/ } \\
\text { ASGA_3DViewe } \\
\text { r/ }\end{array}$ \\
\hline
\end{tabular}

